\title{
CONSTRUCTION OF IRREDUCIBLE REPRESENTATIONS OVER KHOVANOV-LAUDA-ROUQUIER ALGEBRAS OF FINITE CLASSICAL TYPE
}

\author{
GEORGIA BENKART, SEOK-JIN KANG ${ }^{1,2}$, SE-JIN OH ${ }^{3,4}$, AND EUIYONG PARK \\ In Memory of Professor Hyo Chul Myung
}

\begin{abstract}
We give an explicit construction of irreducible modules over Khovanov-LaudaRouquier algebras $R$ and their cyclotomic quotients $R^{\lambda}$ for finite classical types using a crystal basis theoretic approach. More precisely, for each element $v$ of the crystal $B(\infty)$ (resp. $B(\lambda)$ ), we first construct certain modules $\Delta(\mathbf{a} ; k)$ labeled by the adapted string $\mathbf{a}$ of $v$. We then prove that the head of the induced module $\operatorname{Ind}(\Delta(\mathbf{a} ; 1) \nabla \cdots \nabla \Delta(\mathbf{a} ; n))$ is irreducible and that every irreducible $R$-module (resp. $R^{\lambda}$-module) can be realized as the irreducible head of one of the induced modules $\operatorname{Ind}(\Delta(\mathbf{a} ; 1) \otimes \cdots \otimes \Delta(\mathbf{a} ; n))$. Moreover, we show that our construction is compatible with the crystal structure on $B(\infty)$ (resp. $B(\lambda)$ ).
\end{abstract}

\section{INTRODUCTION}

The Khovanov-Lauda-Rouquier algebras (KLR algebras) were introduced independently by Khovanov-Lauda [19, 20] and Rouquier [26] to give a categorification of quantum groups. Let $U_{q}(\mathfrak{g})$ be the quantum group associated with a symmetrizable Cartan datum, and let $R$ be the corresponding KLR algebra. For a dominant integral weight $\lambda$ of $U_{q}(\mathfrak{g})$, the algebra $R$ has a special quotient $R^{\lambda}$ corresponding to $\lambda$, which is called the cyclotomic KhovanovLauda-Rouquier algebra (cyclotomic KLR algebra) of weight $\lambda$. It was conjectured that the cyclotomic quotient $R^{\lambda}$ gives a categorification of the irreducible highest weight $U_{q}(\mathfrak{g})$-module $V(\lambda)$. This was shown for type A in [1, 2, 4]. In [12, Kang and Kashiwara proved this conjecture for all symmetrizable Cartan data. Webster [27] has announced a categorification of tensor products of highest weight modules. In a recent paper, Kang, Oh and Park [14] extended the study of KLR algebras to provide a categorification of quantum generalized Kac-Moody algebras and their crystals. Moreover, Kang, Kashiwara and Oh [13] proved the

2000 Mathematics Subject Classification. 05E10, 16D60, 17B67, 81R10.

Key words and phrases. adapted strings, crystal bases, Khovanov-Lauda-Rouquier algebras.

1 This work was supported by KRF Grant \# 2007-341-C00001.

2 This work was supported by NRF Grant \# 2010-0010753.

3 This work was supported by NRF Grant \# 2010-0019516.

${ }^{4}$ This work was supported by BK21 Mathematical Sciences Division. 
cyclotomic categorification conjecture for irreducible highest weight modules over quantum generalized Kac-Moody algebras.

For symmetrizable Cartan data, the set $\mathbb{B}(\infty)$ (resp. $\mathbb{B}(\lambda)$ ) of isomorphism classes of finitedimensional irreducible graded modules over $R$ (resp. $R^{\lambda}$ ) can be given a crystal structure, and Lauda and Vazirani [22] have shown that there exist crystal isomorphisms $B(\infty) \stackrel{\sim}{\longrightarrow}$ $\mathbb{B}(\infty)$ and $B(\lambda) \stackrel{\sim}{\longrightarrow} \mathbb{B}(\lambda)$, where $B(\infty)$ (resp. $B(\lambda)$ ) is the crystal of $U_{q}^{-}(\mathfrak{g})$ (resp. $V(\lambda)$ ). Kleshchev and Ram [21] gave a construction of irreducible graded $R$-modules for all finite types by using the combinatorics of Lyndon words to construct irreducible $R$-modules as the irreducible heads of the induced modules of the outer tensor products of cuspidal modules. In this approach, the action of Kashiwara operators on the crystal of irreducible modules is hidden in the combinatorics of Lyndon words. Hill, Melvin and Mondragon [7] completed the classification of irreducible $R$-modules begun by Kleshchev and Ram by determining the cuspidal modules. Recently, McNamara 25] investigated this approach using PBW basis theory and computed the global dimension of $R$ for finite type. However, it remains an open problem to construct irreducible graded $R^{\lambda}$-modules. For finite and affine type $A$, results in [1, 3, 10] tell us that the algebra $R^{\lambda}$ has a cellular basis, which yields irreducible modules by using the cellular basis techniques introduced in [6].

In this paper, we give an explicit construction of all irreducible graded modules over $R$ and $R^{\lambda}$ for KLR algebras of finite classical type as the irreducible heads of certain induced modules. This generalizes the type $\mathrm{A}_{n}$ result in [15] to all finite classical types. Our construction differs from the one given by Kleshchev and Ram and is based on the theory of crystal bases. As a result, the action of the Kashiwara operators is an integral part of the construction.

Here is a brief description of our work. Let $\left(\mathfrak{A}, P, \Pi, \mathrm{P}^{\vee}, \Pi^{\vee}\right)$ be a symmetrizable Cartan datum. Let $I$ be the index set of the simple roots, and let $n=|I|$. Set $I_{(n+1)}=I$ and take $I_{(k)}(k=1, \ldots, n)$ to be subsets of $I$ such that $I_{(k)} \subset I_{(k+1)}$ and $\left|I_{(k)}\right|=k$ for all $k$. Let $\mathfrak{B}_{k}$ be the crystal obtained from $B(\infty)$ by forgetting the $i$-arrows for $i \notin I_{(k)}$. For $v \in B(\infty)$, let $u_{0}=v$ and let $u_{k}$ be the highest weight vector of the connected component of $\mathfrak{B}_{k}$ containing $v$ for $k=1, \ldots, n$. Then, there exists a sequence $\mathbf{i}_{k}$ of elements in $I$ such that $u_{k-1}=\tilde{f}_{\mathbf{i}_{k}} u_{k}$, where $\tilde{f}_{\mathbf{i}_{k}}$ is a product of the Kashiwara operators corresponding to the terms in the sequence $\mathbf{i}_{k}$. For $k=1, \ldots, n$, define

$$
\mathcal{N}_{k}(v)=\tilde{f}_{\mathbf{i}_{k}} \mathbf{1}
$$

where 1 is the trivial for the KLR algebra $R(0):=\mathbb{C}$. In Proposition 1.10, we prove for any symmetrizable Cartan datum that hd $\operatorname{lnd}\left(\mathcal{N}_{1}(v) \otimes \cdots \otimes \mathcal{N}_{n}(v)\right)$ is an irreducible graded $R$-module and that the map $\Phi: B(\infty) \longrightarrow \mathbb{B}(\infty)$ defined by

$$
\Phi(v)=\operatorname{hd} \operatorname{lnd}\left(\mathcal{N}_{1}(v) \otimes \cdots \otimes \mathcal{N}_{n}(v)\right) \quad \text { for } v \in B(\infty)
$$


is a crystal isomorphism.

When $\mathfrak{A}$ is of finite type, the modules $\mathcal{N}_{k}(v)$ can be given a more explicit description in terms of the Kashiwara operators $\tilde{f}_{i}$ via the adapted strings introduced by Littelmann in [24]. For this, we choose a special expression $w_{0}=r_{\mathbf{s}_{1}} \cdots r_{\mathbf{s}_{n}}$ of the longest element $w_{0}$ in the Weyl group $W$. Here the $\mathbf{s}_{k}$ are sequences of indices in $I$ (see Table 1). Using the description of adapted strings given in [24], we prove for $v \in B(\infty)$ that

$$
\mathcal{N}_{k}(v)=\tilde{f}_{\mathbf{s}_{k}}^{\mathbf{a}(v)_{k}} \mathbf{1},
$$

where $\mathbf{a}(v)$ is the adapted string of $v$ with respect to this expression for $w_{0}$, and $\mathbf{a}(v)_{k}$ is the subsequence of $\mathbf{a}(v)$ defined by (2.3) (see Proposition 2.3 below).

Our main result is an explicit construction of the irreducible graded modules over the KLR algebras $R$ and $R^{\lambda}$ of finite classical type $\mathrm{A}_{n}, \mathrm{~B}_{n}, \mathrm{C}_{n}, \mathrm{D}_{n}$ in terms of adapted strings. Let $\mathcal{S}$ (resp. $\mathcal{S}^{\lambda}$ ) be the set of adapted strings of $B(\infty)$ (resp. $B(\lambda)$ ) given in Proposition 3.1, and let $\mathbf{B}$ be the crystal of the irreducible module $V\left(\Lambda_{n}\right)$ labeled by the fundamental weight $\Lambda_{n}$ if $\mathfrak{A}$ is of type $\mathrm{A}_{n}$ (resp. $V\left(\Lambda_{1}\right)$ if $\mathfrak{A}$ is of type $\mathbf{B}_{n}, \mathrm{C}_{n}, \mathrm{D}_{n}$ ). For $a, b \in \mathbf{B}$ with $a \succ b$, using the structure of the crystal $\mathbf{B}$, in (3.3) we associate to $a, b$ an irreducible graded module $\Delta_{(a, b)}$. The modules $\Delta_{(a, b)}$ are 1- or 2-dimensional, and in general they are not the cuspidal modules found in [7, 21]. Using the description of $\mathcal{S}$ (resp. $\mathcal{S}^{\lambda}$ ), we define for $v \in B(\infty)$ (resp. $v \in B(\lambda))$ the module $\Delta(\mathbf{a}(v) ; k)$ to be the outer tensor product of modules $\Delta_{(a, b)}$ as in (3.5). Then, it follows from Lemma 4.3 that

$$
\mathcal{N}_{k}(v)=\operatorname{hd} \operatorname{lnd} \Delta(\mathbf{a}(v) ; k) \quad \text { for } k=1, \ldots, n .
$$

The description of the irreducible modules in (0.1) works for all symmetrizable Cartan data. It follows from (0.1) and (0.2) that the irreducible modules for the KLR algebras of finite classical type can be obtained from taking the outer tensor product of heads of induced modules, inducing, then taking the head of the induced module. But this unduly complicated process can be simplified. Indeed, we prove for the module $\Delta(\mathbf{a}(v)):=\Delta(\mathbf{a}(v) ; 1) \otimes \cdots \otimes$ $\Delta(\mathbf{a}(v) ; n)$ that hd $\operatorname{lnd} \Delta(\mathbf{a}(v))$ is irreducible for $v \in B(\infty)$ (resp. $v \in B(\lambda)$ ) for all finite classical types and that the maps

$$
\begin{array}{cl}
\Psi: B(\infty) \longrightarrow \mathbb{B}(\infty) & \text { given by } \Psi(v)=\text { hd Ind } \Delta(\mathbf{a}(v)) \text { for } v \in B(\infty), \\
\Psi^{\lambda}: B(\lambda) \longrightarrow \mathbb{B}(\lambda) & \text { given by } \Psi^{\lambda}(v)=\text { hd Ind } \Delta(\mathbf{a}(v)) \text { for } v \in B(\lambda)
\end{array}
$$

are crystal isomorphisms (Theorem 3.2). Using that fact, we show for the finite classical types that

$$
\begin{aligned}
\mathcal{A} & =\{\operatorname{hd} \operatorname{lnd} \Delta(\mathbf{a}) \mid \mathbf{a} \in \mathcal{S}\} \text { and } \\
\mathcal{A}^{\lambda} & =\left\{\operatorname{hd} \operatorname{lnd} \Delta(\mathbf{a}) \mid \mathbf{a} \in \mathcal{S}^{\lambda}\right\},
\end{aligned}
$$


as a ranges over the adapted strings, provide complete lists of all the irreducible graded modules over $R$ and $R^{\lambda}$, respectively, up to isomorphism and grading shift (Corollary [3.3).

Our paper is organized as follows. Section 1 contains a brief review of crystal bases and KLR algebras associated with any symmetrizable Cartan datum. This section culminates with the proof of the crystal isomorphism $\Phi: B(\infty) \longrightarrow \mathbb{B}(\infty)$ in (0.1). Section 2 specializes to the case of finite type, first reviewing Littelmann's result (see 24]) on adapted stings and then giving an explicit description of the modules $\mathcal{N}_{k}(v)$ for all finite types via adapted stings. Combining this description with the definition of $\mathcal{N}_{k}(v)$, we obtain an expression for $\mathcal{N}_{k}(v)$ in terms of Kashiwara operators.

In Section 3, we restrict to the case of finite classical types. We first define the modules $\Delta_{(a, b)}$ using the structure of the crystals $\mathbf{B}$ and construct the module $\Delta(\mathbf{a} ; k)$ for $\mathbf{a} \in \mathcal{S}$ (resp. $\mathbf{a} \in \mathcal{S}^{\lambda}$ ) as an outer tensor product of modules $\Delta_{(a, b)}$ in (3.5). We then construct the maps $\Psi: B(\infty) \longrightarrow \mathbb{B}(\infty)$ and $\Psi^{\lambda}: B(\lambda) \longrightarrow \mathbb{B}(\lambda)$ by taking the head of Ind $\Delta(\mathbf{a}(v))$ for $v \in B(\infty)$ (resp. $v \in B(\lambda)$ ). We illustrate this construction by presenting an example for type $\mathrm{B}_{3}$ using the Kashiwara-Nakashima tableaux in [18] to realize the crystal $B(\lambda)$. We prove in Proposition 3.4 for any finite classical type and any $v \in B(\lambda)$ that the number $\eta(v)$ of $\Delta_{(a, b)}$ 's in $\Delta(\mathbf{a}(v))$ has an upper bound; i.e., $\eta(v) \leq n \lambda(h)$ for a certain element $h \in \mathrm{P}^{\vee}$ (which depends on the type).

Section 4 is devoted to proving that the maps $\Psi: B(\infty) \longrightarrow \mathbb{B}(\infty)$ and $\Psi^{\lambda}: B(\lambda) \longrightarrow \mathbb{B}(\lambda)$ are crystal isomorphisms. To accomplish this, we give a sufficient condition in Lemma 4.1 for the isomorphism $\operatorname{Ind}\left(\Delta_{(a, b)} \otimes \Delta_{(c, d)}\right) \simeq \operatorname{lnd}\left(\Delta_{(c, d)} \otimes \Delta_{(a, b)}\right)$ to exist for finite classical types. Using this condition together with Lemma 4.3 of [15] and the surjective homomorphism $\operatorname{Ind}\left(\Delta_{(a, b)} \otimes \Delta_{(b, c)}\right) \rightarrow \Delta_{(a, c)}$, we prove in Lemma 4.3 that $\mathcal{N}_{n}(v)=\operatorname{hd} \operatorname{Ind} \Delta(\mathbf{a}(v) ; n)$. It follows from that result and the choice of the sequence corresponding to $k$ that $\mathcal{N}_{k}(v)=$ hd $\operatorname{lnd} \Delta(\mathbf{a}(v) ; k)$ for all $k=1, \ldots, n$. Combining this with the crystal isomorphism $\Phi$ in (0.1), we establish the crystal isomorphisms $\Psi: B(\infty) \longrightarrow \mathbb{B}(\infty)$ and $\Psi^{\lambda}: B(\lambda) \longrightarrow \mathbb{B}(\lambda)$. This then gives an explicit realization compatible with the Kashiwara operators of all the irreducible graded modules over $R$ and $R^{\lambda}$ for finite classical types.

\section{Crystals and Khovanov-Lauda-Rouquier Algebras}

\subsection{Crystals.}

Let $I$ be a finite index set. A square matrix $\mathfrak{A}=\left(a_{i j}\right)_{i, j \in I}$ is a symmetrizable generalized Cartan matrix if it satisfies (i) $a_{i i}=2$ for $i \in I$, (ii) $a_{i j} \in \mathbb{Z}_{\leq 0}$ for $i \neq j$, (iii) $a_{i j}=0$ if $a_{j i}=0$ for $i, j \in I$, (iv) there is a diagonal matrix $\mathfrak{D}=\operatorname{diag}\left(\delta_{i} \in \mathbb{Z}_{>0} \mid i \in I\right)$ such that $\mathfrak{D A}$ is symmetric.

A Cartan datum $\left(\mathfrak{A}, \mathrm{P}, \Pi, \mathrm{P}^{\vee}, \Pi^{\vee}\right)$ consists of

(1) a symmetrizable generalized Cartan matrix $\mathfrak{A}$, 
(2) a free abelian group $\mathrm{P}$ of finite rank, called the weight lattice,

(3) the set $\Pi=\left\{\alpha_{i} \mid i \in I\right\} \subset \mathrm{P}$ of simple roots,

(4) the dual weight lattice $\mathrm{P}^{\vee}:=\operatorname{Hom}(\mathrm{P}, \mathbb{Z})$,

(5) the set $\Pi^{\vee}=\left\{h_{i} \mid i \in I\right\} \subset \mathrm{P}^{\vee}$ of simple coroots,

which satisfy the following properties:

(i) $\left\langle h_{i}, \alpha_{j}\right\rangle:=\alpha_{j}\left(h_{i}\right)=a_{i j}$ for all $i, j \in I$,

(ii) $\Pi \subset \mathfrak{h}^{*}$ is linearly independent, where $\mathfrak{h}:=\mathbb{C} \otimes_{\mathbb{Z}} \mathrm{P}^{\vee}$ and $\mathfrak{h}^{*}$ is the dual space,

(iii) for each $i \in I$, there exists $\Lambda_{i} \in \mathrm{P}$ such that $\left\langle h_{j}, \Lambda_{i}\right\rangle=\delta_{i j}$ for all $j \in I$.

The $\Lambda_{i}$ are the fundamental weights. We denote by $\mathrm{P}^{+}=\left\{\lambda \in \mathrm{P} \mid \lambda\left(h_{i}\right) \in \mathbb{Z}_{\geq 0}, i \in I\right\}$ the set of dominant integral weights. The free abelian group $\mathrm{Q}=\bigoplus_{i \in I} \mathbb{Z} \alpha_{i}$ is the root lattice, and $\mathrm{Q}^{+}=\sum_{i \in I} \mathbb{Z}_{\geq 0} \alpha_{i}$ is the positive root lattice. For $\alpha=\sum_{i \in I} k_{i} \alpha_{i} \in \mathrm{Q}^{+}$, the height of $\alpha$ is $|\alpha|:=\sum_{i \in I} k_{i}$. There is a symmetric bilinear form $(\mid)$ on $\mathfrak{h}^{*}$ such that

$$
\left(\alpha_{i} \mid \alpha_{j}\right)=\delta_{i} a_{i j} \text { for } i, j \in I, \quad\left\langle h_{i}, \lambda\right\rangle=\frac{2\left(\alpha_{i} \mid \lambda\right)}{\left(\alpha_{i} \mid \alpha_{i}\right)} \text { for } \lambda \in \mathfrak{h}^{*} \text { and } i \in I .
$$

Let $W$ be the Weyl group, which is the subgroup of Aut $\left(\mathfrak{h}^{*}\right)$ generated by simple reflections $\left\{r_{i}\right\}_{i \in I}$ defined by $r_{i}(\lambda):=\lambda-\left\langle h_{i}, \lambda\right\rangle \alpha_{i}$ for $\lambda \in \mathfrak{h}^{*}$ and $i \in I$.

Let $q$ be an indeterminate. For $i \in I$ and $m, n \in \mathbb{Z}_{\geq 0}$, define

$$
q_{i}=q^{\delta_{i}}, \quad[n]_{q_{i}}=\frac{q_{i}{ }^{n}-q_{i}{ }^{-n}}{q_{i}-q_{i}{ }^{-1}}, \quad[n]_{q_{i}} !=\prod_{k=1}^{n}[k]_{q_{i}}, \quad\left[\begin{array}{c}
m \\
n
\end{array}\right]_{q_{i}}=\frac{[m]_{q_{i}} !}{[m-n]_{q_{i}} ![n]_{q_{i}} !} .
$$

Definition 1.1. The quantum group $U_{q}(\mathfrak{g})$ associated with the Cartan datum $\left(\mathfrak{A}, \mathrm{P}, \Pi, \mathrm{P}^{\vee}, \Pi^{\vee}\right)$ is the associative algebra over $\mathbb{Q}(q)$ with 1 generated by $e_{i}, f_{i}(i \in I)$ and $q^{h}\left(h \in \mathrm{P}^{\vee}\right)$ satisfying the following relations:

(1) $q^{0}=1, q^{h} q^{h^{\prime}}=q^{h+h^{\prime}}$ for $h, h^{\prime} \in \mathrm{P}^{\vee}$,

(2) $q^{h} e_{i} q^{-h}=q^{\left\langle h, \alpha_{i}\right\rangle} e_{i}, q^{h} f_{i} q^{-h}=q^{-\left\langle h, \alpha_{i}\right\rangle} f_{i}$ for $h \in \mathrm{P}^{\vee}, i \in I$,

(3) $e_{i} f_{j}-f_{j} e_{i}=\delta_{i j} \frac{K_{i}-K_{i}^{-1}}{q_{i}-q_{i}^{-1}}, \quad$ where $K_{i}=q^{\delta_{i} h_{i}}$

(4) $\sum_{k=0}^{1-a_{i j}}(-1)^{k}\left[\begin{array}{c}1-a_{i j} \\ k\end{array}\right]_{q_{i}} e_{i}^{1-a_{i j}-k} e_{j} e_{i}^{k}=0 \quad$ if $i \neq j$,

(5) $\sum_{k=0}^{1-a_{i j}}(-1)^{k}\left[\begin{array}{c}1-a_{i j} \\ k\end{array}\right]_{q_{i}} f_{i}^{1-a_{i j}-k} f_{j} f_{i}^{k}=0 \quad$ if $i \neq j$.

The definition of the category $O_{i n t}^{q}$ of integrable $U_{q}(\mathfrak{g})$-modules, crystal bases, and Kashiwara operators can be found, for example, in [8, 16. It was proved in [16] that every $U_{q}(\mathfrak{g})$ module in the category $O_{\text {int }}^{q}$ has a unique crystal basis $(L, B)$. Let us recall the notion of a crystal as defined in [17]. 
Definition 1.2. A crystal is a set $B$ together with maps wt $: B \rightarrow \mathrm{P}, \varphi_{i}, \varepsilon_{i}: B \rightarrow$ $\mathbb{Z} \sqcup\{-\infty\}$ and $\tilde{e}_{i}, \tilde{f}_{i}: B \rightarrow B \sqcup\{0\}(i \in I)$ which satisfy the following conditions:

(1) $\varphi_{i}(b)=\varepsilon_{i}(b)+\left\langle h_{i}, w \mathrm{wt}(b)\right\rangle$,

(2) $\operatorname{wt}\left(\tilde{e}_{i} b\right)=\operatorname{wt}(b)+\alpha_{i}, \quad \operatorname{wt}\left(\tilde{f}_{i} b\right)=\operatorname{wt}(b)-\alpha_{i}$ if $\tilde{e}_{i} b, \tilde{f}_{i} b \in B$,

(3) for $b, b^{\prime} \in B$ and $i \in I, b^{\prime}=\tilde{e}_{i} b$ if and only if $b=\tilde{f}_{i} b^{\prime}$,

(4) for $b \in B$, if $\varphi_{i}(b)=-\infty$, then $\tilde{e}_{i} b=\tilde{f}_{i} b=0$,

(5) if $b \in B$ and $\tilde{e}_{i} b \in B$, then $\varepsilon_{i}\left(\tilde{e}_{i} b\right)=\varepsilon_{i}(b)-1, \quad \varphi_{i}\left(\tilde{e}_{i} b\right)=\varphi_{i}(b)+1$,

(6) if $b \in B$ and $\tilde{f}_{i} b \in B$, then $\varepsilon_{i}\left(\tilde{f}_{i} b\right)=\varepsilon_{i}(b)+1, \quad \varphi_{i}\left(\tilde{f}_{i} b\right)=\varphi_{i}(b)-1$.

For $\mathbf{i}=\left(i_{1}, \ldots, i_{m}\right) \in I^{m}$ and $\mathbf{k}=\left(k_{1}, \ldots, k_{m}\right) \in\left(\mathbb{Z}_{\geq 0}\right)^{m}$, let $\tilde{f}_{\mathbf{i}}^{\mathbf{k}}=\tilde{f}_{i_{1}}^{k_{1}} \cdots \tilde{f}_{i_{m}}^{k_{m}}$ (resp. $\left.\tilde{e}_{\mathbf{i}}^{\mathbf{k}}=\tilde{e}_{i_{1}}^{k_{1}} \cdots \tilde{e}_{i_{m}}^{k_{m}}\right)$. If $\mathbf{k}=(1, \ldots, 1)$, then we write $\tilde{f}_{\mathbf{i}}\left(\operatorname{resp} . \tilde{e}_{\mathbf{i}}\right)$ for $\tilde{f}_{\mathbf{i}}^{\mathbf{k}}\left(\operatorname{resp} . \tilde{e}_{\mathbf{i}}^{\mathbf{k}}\right)$.

\section{Examples 1.3.}

(1) Associated with each module $M \in O_{i n t}^{q}$ is a crystal basis $(L, B)$, where $B$ is a crystal with the maps

$$
\varepsilon_{i}(b)=\max \left\{k \geq 0 \mid \tilde{e}_{i}^{k} b \neq 0\right\}, \quad \varphi_{i}(b)=\max \left\{k \geq 0 \mid \tilde{f}_{i}^{k} b \neq 0\right\} .
$$

We denote by $B(\lambda)$ the crystal of the irreducible highest weight module $V(\lambda) \in O_{\text {int }}^{q}$ with highest weight $\lambda \in \mathrm{P}^{+}$and write $b_{\lambda}$ for the highest weight element of $B(\lambda)$.

(2) Let $(L(\infty), B(\infty))$ be the crystal basis of $U_{q}^{-}(\mathfrak{g})$. Then $B(\infty)$ is a crystal with the maps given by

$$
\varepsilon_{i}(b)=\max \left\{k \geq 0 \mid \tilde{e}_{i}^{k} b \neq 0\right\}, \quad \varphi_{i}(b)=\varepsilon_{i}(b)+\left\langle h_{i}, \mathrm{wt}(b)\right\rangle .
$$

Let 1 denote the highest weight element of $B(\infty)$.

(3) For $\lambda \in \mathrm{P}$, the set $T_{\lambda}=\left\{t_{\lambda}\right\}$ is a crystal with

$$
\begin{aligned}
& \operatorname{wt}\left(t_{\lambda}\right)=\lambda, \quad \tilde{e}_{i} t_{\lambda}=\tilde{f}_{i} t_{\lambda}=0 \text { for } i \in I, \\
& \varepsilon_{i}\left(t_{\lambda}\right)=\varphi_{i}\left(t_{\lambda}\right)=-\infty \text { for } i \in I .
\end{aligned}
$$

(4) The set $C=\{c\}$ is a crystal with

$$
\operatorname{wt}(c)=0, \quad \tilde{e}_{i} c=\tilde{f}_{i} c=0, \quad \varepsilon_{i}(c)=\varphi_{i}(c)=0 \quad(i \in I) .
$$

We refer to [8, 17] for more details on $U_{q}(\mathfrak{g})$-crystals. It was shown in [11, 17] that for each $\lambda \in \mathrm{P}^{+}$there is a unique strict crystal embedding

$$
\iota_{\lambda}: B(\lambda) \rightarrow B(\infty) \otimes T_{\lambda} \otimes C
$$

sending $b_{\lambda}$ to $1 \otimes t_{\lambda} \otimes c$. 


\subsection{Khovanov-Lauda-Rouquier algebras.}

For $\alpha \in \mathrm{Q}^{+}$with $|\alpha|=m$, we define $I^{\alpha}=\left\{\mathbf{i}=\left(i_{1}, \ldots, i_{m}\right) \in I^{m} \mid \sum_{k=1}^{m} \alpha_{i_{k}}=\alpha\right\}$. Given $\mathbf{i}=\left(i_{1}, \ldots, i_{m}\right) \in I^{\alpha}$ and $\mathbf{j}=\left(j_{1}, \ldots, j_{m^{\prime}}\right) \in I^{\beta}$, let $\mathbf{i} * \mathbf{j}$ denote the concatenation of $\mathbf{i}$ and $\mathbf{j}: \mathbf{i} * \mathbf{j}=\left(i_{1}, \ldots, i_{m}, j_{1}, \ldots, j_{m^{\prime}}\right) \in I^{\alpha+\beta}$. Let $S_{m}$ be the symmetric group on $m$ letters with simple transpositions $\sigma_{i}(i=1, \ldots, m-1)$. Then $S_{m}$ acts on $I^{m}$ in a natural way. For $d_{1}, \ldots, d_{n} \in \mathbb{Z}_{\geq 0}$, we denote by $S_{d_{1}+\cdots+d_{n}} / S_{d_{1}} \times \cdots \times S_{d_{n}}$ the set of minimal left coset representatives of $S_{d_{1}} \times \cdots \times S_{d_{n}}$ in $S_{d_{1}+\cdots+d_{n}}$.

Let $\mathrm{u}, \mathrm{v}$ be indeterminates. For each $i, j \in I$, we choose $\zeta_{i j} \in \mathbb{C} \backslash\{0\}$ such that $\zeta_{i j}=\zeta_{j i}$ if $a_{i j}=0$ and elements $\eta_{i j} \in \mathbb{C}$ with $\eta_{i j}^{p q}=\eta_{j i}^{q p}$ for all $p, q \in \mathbb{Z}_{>0}$ such that $\delta_{i} p+\delta_{j} q=-\left(\alpha_{i} \mid \alpha_{j}\right)$ and set

$$
\mathcal{Q}_{i j}(\mathrm{u}, \mathrm{v})= \begin{cases}0 & \text { if } i=j, \\ \zeta_{i j} & \text { if } i \neq j, a_{i j}=0, \\ \zeta_{i j} \mathbf{u}^{-a_{i j}}+\sum_{\substack{p, q>0, \delta_{i} p+\delta_{j} q=-\left(\alpha_{i} \mid \alpha_{j}\right)}} \eta_{i j}^{p q} \mathbf{u}^{p} \mathbf{v}^{q}+\zeta_{j i} \mathbf{v}^{-a_{j i}} & \text { otherwise. }\end{cases}
$$

\section{Definition 1.4.}

(1) Let $\alpha \in \mathrm{Q}^{+}$with $|\alpha|=m$. The homogeneous Khovanov-Lauda-Rouquier algebra $R(\alpha)$ at $\alpha$ associated with $\mathfrak{A}$ and $\left(\mathcal{Q}_{i j}\right)_{i, j \in I}$ is the associative graded $\mathbb{C}$-algebra generated by $e(\mathbf{i})\left(\mathbf{i}=\left(i_{1}, \ldots, i_{m}\right) \in I^{\alpha}\right), x_{\ell}(1 \leq \ell \leq m), \tau_{k}(1 \leq k<m)$ satisfying the following defining relations:

$$
\begin{aligned}
& e(\mathbf{i}) e(\mathbf{j})=\delta_{\mathbf{i}, \mathbf{j}} e(\mathbf{i}), \quad \sum_{\mathbf{i} \in I^{\alpha}} e(\mathbf{i})=1, \quad x_{k} x_{\ell}=x_{\ell} x_{k}, \quad x_{\ell} e(\mathbf{i})=e(\mathbf{i}) x_{\ell}, \\
& \tau_{k} e(\mathbf{i})=e\left(\sigma_{k}(\mathbf{i})\right) \tau_{k}, \quad \tau_{k} \tau_{\ell}=\tau_{\ell} \tau_{k} \quad \text { if }|k-\ell|>1, \\
& \tau_{k}^{2} e(\mathbf{i})=\mathcal{Q}_{i_{k}, i_{k+1}}\left(x_{k}, x_{k+1}\right) e(\mathbf{i}), \\
& \left(\tau_{k} x_{\ell}-x_{\sigma_{k}(\ell)} \tau_{k}\right) e(\mathbf{i})= \begin{cases}-e(\mathbf{i}) & \text { if } \ell=k, \quad i_{k}=i_{k+1}, \\
e(\mathbf{i}) & \text { if } \ell=k+1, \quad i_{k}=i_{k+1}, \\
0 & \text { otherwise, }\end{cases} \\
& \left(\tau_{k+1} \tau_{k} \tau_{k+1}-\tau_{k} \tau_{k+1} \tau_{k}\right) e(\mathbf{i}) \\
& = \begin{cases}\frac{\mathcal{Q}_{i_{k}, i_{k+1}}\left(x_{k+2}, x_{k+1}\right)-\mathcal{Q}_{i_{k}, i_{k+1}}\left(x_{k}, x_{k+1}\right)}{x_{k+2}-x_{k}} e(\mathbf{i}) & \text { if } i_{k}=i_{k+2} \neq i_{k+1}, \\
0 & \text { otherwise. }\end{cases}
\end{aligned}
$$

The algebra

$$
R:=\bigoplus_{\alpha \in Q^{+}} R(\alpha)
$$


is called the Khovanov-Lauda-Rouquier algebra (KLR algebra) associated with $\mathfrak{A}$ and $\left(\mathcal{Q}_{i j}\right)_{i, j \in I}$.

(2) Let $\lambda \in \mathrm{P}^{+}$. The homogeneous cyclotomic Khovanov-Lauda-Rouquier algebra $R^{\lambda}(\alpha)$ at $\alpha$ of weight $\lambda$ is the quotient algebra of $R(\alpha)$ by the two-sided ideal $I^{\lambda}(\alpha)$ of $R(\alpha)$ generated by $x_{m}^{\left\langle h_{i_{m}}, \lambda\right\rangle} e(\mathbf{i})\left(\mathbf{i} \in I^{\alpha}\right)$. The algebra

$$
R^{\lambda}:=\bigoplus_{\alpha \in Q^{+}} R^{\lambda}(\alpha)
$$

is the cyclotomic Khovanov-Lauda-Rouquier algebra (cyclotomic KLR algebra) of weight $\lambda$.

The $\mathbb{Z}$-grading on $R(\alpha)$ is given by

$$
\operatorname{deg}(e(\mathbf{i}))=0, \quad \operatorname{deg}\left(x_{\ell} e(\mathbf{i})\right)=\left(\alpha_{i_{\ell}} \mid \alpha_{i_{\ell}}\right), \quad \operatorname{deg}\left(\tau_{k} e(\mathbf{i})\right)=-\left(\alpha_{i_{k}} \mid \alpha_{i_{k+1}}\right) .
$$

Here $R(0)=\mathbb{C}$. Let $R(\alpha)$-fmod (resp. $R^{\lambda}(\alpha)$-fmod) be the category of finite-dimensional $\mathbb{Z}$-graded $R(\alpha)$-modules (resp. $R^{\lambda}(\alpha)$-modules). Any $N \in R^{\lambda}(\alpha)$-fmod can be viewed as a graded $R(\alpha)$-module annihilated by $I^{\lambda}(\alpha)$. We write infl${ }^{\lambda} N$ when considering $N \in R^{\lambda}(\alpha)$ fmod as an $R(\alpha)$-module. Any $M \in R(\alpha)$-fmod gives rise to the $R^{\lambda}(\alpha)$-module $\operatorname{pr}^{\lambda} M:=$ $M / I^{\lambda}(\alpha) M$. From now on, when there is no possibility of confusion, we identify irreducible graded $R^{\lambda}(\alpha)$-modules with irreducible graded $R(\alpha)$-modules annihilated by $I^{\lambda}(\alpha)$ via infl${ }^{\lambda}$.

Let

$$
G_{0}(R)=\bigoplus_{\alpha \in Q^{+}} G_{0}(R(\alpha) \text {-fmod }), \quad G_{0}\left(R^{\lambda}\right)=\bigoplus_{\alpha \in Q^{+}} G_{0}\left(R^{\lambda}(\alpha) \text {-fmod }\right),
$$

where $G_{0}\left(R(\alpha)\right.$-fmod) (resp. $G_{0}\left(R^{\lambda}(\alpha)\right.$-fmod)) is the Grothendieck group of $R(\alpha)$-fmod (resp. of $R^{\lambda}(\alpha)$-fmod). For $M \in R(\alpha)$-fmod (resp. $R(\alpha)^{\lambda}$-fmod), $[M]$ stands for the isomorphism class of $M$ in $G_{0}(R(\alpha))$ (resp. $G_{0}\left(R^{\lambda}(\alpha)\right)$ ). When no confusion can arise, we write $M$ for $[M]$.

Given $M=\bigoplus_{i \in \mathbb{Z}} M_{i}$, let $M\langle k\rangle=\bigoplus_{i \in \mathbb{Z}} M\langle k\rangle_{i}$ denote the graded module obtained from $M$ by shifting the grading by $k$, where $M\langle k\rangle_{i}:=M_{i+k}$ for $i \in \mathbb{Z}$. The $q$-character $\operatorname{ch}_{q}(M)$ and character $\operatorname{ch}(M)$ of $M$ are defined by

$$
\operatorname{ch}_{q}(M):=\sum_{\mathbf{i} \in I^{\alpha}} \operatorname{dim}_{q}(e(\mathbf{i}) M) \mathbf{i}, \quad \operatorname{ch}(M):=\sum_{\mathbf{i} \in I^{\alpha}} \operatorname{dim}(e(\mathbf{i}) M) \mathbf{i}
$$

where $\operatorname{dim}_{q}(N):=\sum_{i \in \mathbb{Z}}\left(\operatorname{dim} N_{i}\right) q^{i}$ for any graded module $N=\bigoplus_{i \in \mathbb{Z}} N_{i}$. For $\mathbf{i} \in I^{\alpha}$, we write $\mathbf{i} \in \operatorname{ch}(M)\left(\right.$ resp. $\left.\mathbf{i} \in \operatorname{ch}_{q}(M)\right)$ when $\mathbf{i}$ occurs in $\operatorname{ch} M\left(\right.$ resp. $\left.\operatorname{ch}_{q}(M)\right)$ with a nonzero coefficient.

Notation and Conventions. In this paper, by a homomorphism we mean a homogeneous homomorphism of some degree $k \in \mathbb{Z}$. The symbol $\simeq($ resp. $\rightarrow, \hookrightarrow$ ) will be used to denote 
an isomorphism (resp. a surjective homomorphism, an injective homomorphism) up to a grading shift, and the notation $\cong$ will be reserved for a degree preserving isomorphism. For $M \in R(\alpha)$-fmod and $N \in R(\beta)$-fmod, $M \otimes N$ will denote the outer tensor product of $M$ and $N$.

For $M, N \in R(\alpha)$-fmod, let $\operatorname{Hom}(M, N)$ be the $\mathbb{C}$-vector space of homogeneous homomorphisms of degree 0 , and let $\operatorname{HOM}(M, N)=\bigoplus_{k \in \mathbb{Z}} \operatorname{Hom}(M, N\langle k\rangle)$. For $\beta_{1}, \ldots, \beta_{m} \in \mathrm{Q}^{+}$, we define

$$
e\left(\beta_{1}, \ldots, \beta_{m}\right)=\sum_{\mathbf{i}_{j} \in I^{\beta_{j}}} e\left(\mathbf{i}_{1} * \cdots * \mathbf{i}_{m}\right) .
$$

The natural embedding $R\left(\beta_{1}\right) \otimes \cdots \otimes R\left(\beta_{m}\right) \hookrightarrow R\left(\beta_{1}+\cdots+\beta_{m}\right)$ gives the following functors:

$$
\begin{aligned}
\operatorname{Ind}_{\beta_{1}, \ldots, \beta_{m}}- & :=R\left(\beta_{1}+\cdots+\beta_{m}\right) \otimes_{R\left(\beta_{1}\right) \otimes \cdots \otimes R\left(\beta_{m}\right)}, \\
\operatorname{Coind}_{\beta_{1}, \ldots, \beta_{m}}- & :=\operatorname{HOM}_{R\left(\beta_{1}\right) \otimes \cdots \otimes R\left(\beta_{m}\right)}\left(R\left(\beta_{1}+\cdots+\beta_{m}\right), \ldots\right), \\
\operatorname{Res}_{\beta_{1}, \ldots, \beta_{m}}- & :=e\left(\beta_{1}, \ldots, \beta_{m}\right) \_
\end{aligned}
$$

Lemma $1.5(22]) . \quad$ (1) Let $M_{k} \in R\left(\beta_{k}\right)$-fmod for $k=1, \ldots, m$. Then

$$
\operatorname{lnd}_{\beta_{1}, \ldots, \beta_{m}}\left(M_{1} \otimes \cdots \otimes M_{m}\right) \simeq \operatorname{Coind}_{\beta_{m}, \ldots, \beta_{1}}\left(M_{m} \otimes \cdots \otimes M_{1}\right) .
$$

(2) For $M \in R\left(\beta_{1}\right) \otimes \cdots \otimes R\left(\beta_{m}\right)$-fmod and $N \in R\left(\beta_{1}+\cdots+\beta_{k}\right)$-fmod,

$$
\begin{aligned}
\operatorname{HOM}_{R\left(\beta_{1}+\cdots+\beta_{m}\right)}\left(\operatorname{Ind}_{\beta_{1}, \ldots \beta_{m}} M, N\right) & \cong \operatorname{HOM}_{R\left(\beta_{1}\right) \otimes \cdots \otimes R\left(\beta_{m}\right)}\left(M, \operatorname{Res}_{\beta_{1}, \ldots, \beta_{m}} N\right), \\
\operatorname{HOM}_{R\left(\beta_{1}+\cdots+\beta_{m}\right)}\left(N, \operatorname{Coind}_{\beta_{1}, \ldots, \beta_{m}} M\right) & \cong \operatorname{HOM}_{R\left(\beta_{1}\right) \otimes \cdots \otimes R\left(\beta_{m}\right)}\left(\operatorname{Res}_{\beta_{1}, \ldots, \beta_{m}} N, M\right) .
\end{aligned}
$$

For $m \in \mathbb{Z}_{\geq 0}$ and $i \in I$, let

$$
L\left(i^{m}\right)=\operatorname{lnd}_{\mathbb{C}\left[x_{1}, \ldots, x_{m}\right]}^{R\left(m \alpha_{i}\right)} \phi,
$$

where $\phi$ is the 1 -dimensional trivial module over $\mathbb{C}\left[x_{1}, \ldots, x_{m}\right]$ with $x_{j} \phi=0$ for all $j=$ $1, \ldots, m$, and $\operatorname{dim}_{q}(\phi)=1$. Let $\mathbb{B}(\infty)$ (resp. $\mathbb{B}(\lambda)$ ) be the set of all isomorphism classes of irreducible graded $R$-modules (resp. $R^{\lambda}$-modules). Let 1 denote the 1-dimensional trivial $R(0)$-module. For a graded $R(\beta)$-module (resp. $R^{\lambda}(\beta)$-module) $M$, it was shown in [12, 19, 22 ] that the operators defined by

$$
\begin{aligned}
& e_{i}(M)=\operatorname{Res}_{\beta-\alpha_{i}}^{\alpha_{i}, \beta-\alpha_{i}}\left(e\left(\alpha_{i}, \beta-\alpha_{i}\right) M\right), \\
& f_{i}(M)=\left\{\begin{array}{lll}
R\left(\beta+\alpha_{i}\right) e\left(\alpha_{i}, \beta\right) \otimes_{R(\beta)} M & \text { if } & M \in R(\beta) \text {-mod, } \\
R^{\lambda}\left(\beta+\alpha_{i}\right) e\left(\alpha_{i}, \beta\right) \otimes_{R^{\lambda}(\beta)} M & \text { if } & M \in R^{\lambda}(\beta) \text {-mod. }
\end{array}\right.
\end{aligned}
$$

satisfy

$$
\sum_{k=0}^{1-a_{i j}}(-1)^{k} e_{i}^{\left(1-a_{i j}-k\right)} e_{j} e_{i}^{(k)}[M]=0, \quad \sum_{k=0}^{1-a_{i j}}(-1)^{k} f_{i}^{\left(1-a_{i j}-k\right)} f_{j} f_{i}^{(k)}[M]=0
$$


where $e_{i}^{(r)}[M]:=\frac{1}{[r]_{q_{i}} !}\left[e_{i}^{r} M\right]$ and $f_{i}^{(r)}[M]:=\frac{1}{[r]_{q_{i}} !}\left[f_{i}^{r} M\right]$ for $i, j \in I, r \in \mathbb{Z}_{\geq 0}$.

We define

$$
\begin{aligned}
& \operatorname{wt}(M)= \begin{cases}-\beta & \text { if } M \in R(\beta) \text {-fmod, } \\
\lambda-\beta & \text { if } M \in R^{\lambda}(\beta) \text {-fmod, }\end{cases} \\
& \varepsilon_{i}(M)=\max \left\{k \geq 0 \mid e_{i}^{k} M \neq 0\right\}, \quad \varphi_{i}(M)=\varepsilon_{i}(M)+\operatorname{wt}(M)\left(h_{i}\right), \\
& \tilde{e}_{i}(M)=\operatorname{soc}\left(e_{i} M\right), \text { and } \tilde{f}_{i}(M)= \begin{cases}\operatorname{hdlnd}_{\alpha_{i}, \beta}(L(i) \otimes M) & \text { if } M \in R(\beta) \text {-fmod, } \\
\operatorname{pr}^{\lambda} \circ \tilde{f}_{i} \circ \operatorname{infl}^{\lambda} M & \text { if } M \in R^{\lambda}(\beta) \text {-fmod. }\end{cases}
\end{aligned}
$$

The Kashiwara operators $\tilde{e}_{i}$ and $\tilde{f}_{i}$ are defined in the opposite manner in [15, 19]. We have made this change so they can be viewed as operators acting on the left of irreducible modules. In Section 3, we will explain further why we want the Kashiwara operators defined in this way.

Theorem 1.6 ([22, Thm. 7.4, Thm. 7.5]). The sextuple $\left(\mathbb{B}(\infty)\right.$, wt, $\left.\tilde{e}_{i}, \tilde{f}_{i}, \varepsilon_{i}, \varphi_{i}\right)$ (resp. $\left.\left(\mathbb{B}(\lambda), \mathrm{wt}, \tilde{e}_{i}, \tilde{f}_{i}, \varepsilon_{i}, \varphi_{i}\right)\right)$ is a crystal, which is isomorphic to the crystal $B(\infty)$ (resp. $B(\lambda)$ ).

\subsection{Construction of irreducible $R$-modules.}

In this subsection, we give a construction of irreducible graded $R$-modules as the irreducible heads of certain induced modules, which is compatible with the Kashiwara operators. More precisely, set $I_{(n+1)}=I$ and let $I_{(k)} \subset I(k=1, \ldots, n)$ be subsets of $I$ such that $I_{(k)} \subseteq I_{(k+1)}$ and $\left|I_{(k)}\right|=k$. Let $\mathfrak{B}_{k}$ be the crystal obtained from $B(\infty)$ by forgetting the $i$-arrows for $i \notin I_{(k)}$. Using highest weight vectors of connected components of $\mathfrak{B}_{k}$, we define irreducible $R$-modules $\mathcal{N}_{k}(v)$ for $k=1, \ldots, n$ and $v \in B(\infty)$. Then, we construct a crystal isomorphism $\Phi: B(\infty) \rightarrow \mathbb{B}(\infty)$ defined by $\Phi(v)=$ hd Ind $\left(\mathcal{N}_{1}(v) \otimes \cdots \otimes \mathcal{N}_{n}(v)\right.$ ) (Proposition 1.10). We emphasize that this crystal isomorphism $\Phi$ exists for an arbitrary symmetrizable Cartan datum.

For $M \in R(\beta)$-fmod, $M_{k} \in R\left(\beta_{k}\right)$-fmod $(k=1, \ldots, m)$ and $d \in \mathbb{Z}_{>0}$, set

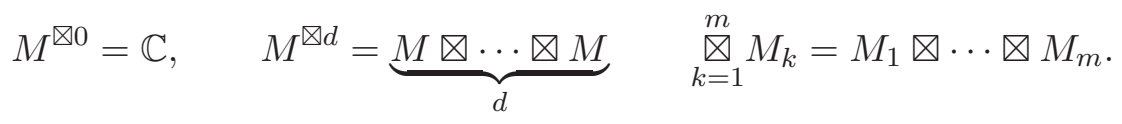

Lemma 1.7. Let $M_{k} \in R\left(\beta_{k}\right)$-fmod, $\quad d_{k} \in \mathbb{Z}_{\geq 0}$, and $\mathbf{i}_{k} \in I^{d_{k} \beta_{k}}$ for $k=1, \ldots, m$. Suppose that $\mathbf{i}_{k}$ occurs in $\operatorname{ch} \operatorname{Ind}\left(M_{k}^{\bigotimes d_{k}}\right)$ with multiplicity $\xi_{k} \in \mathbb{Z}_{>0}$ for $k=1, \ldots, m$. Assume

(i) Ind $\left(M_{k} \otimes M_{k^{\prime}}\right) \simeq \operatorname{Ind}\left(M_{k^{\prime}} \otimes M_{k}\right)$ for $k, k^{\prime}=1, \ldots, m$,

(ii) Ind $\left(M_{k}^{\bigotimes d_{k}}\right)$ is irreducible for $k=1, \ldots, m$, and

(iii) $\mathbf{i}:=\mathbf{i}_{1} * \cdots * \mathbf{i}_{m}$ occurs in ch Ind $\left(\underset{k=1}{\otimes} M_{k}^{\bigotimes d_{k}}\right)$ with multiplicity $\xi_{1} \xi_{2} \cdots \xi_{m}$. 
Then Ind $\left(\underset{k=1}{\otimes} M_{k}^{\bigotimes d_{k}}\right)$ is irreducible.

Proof. Let $N_{k}=\operatorname{Ind}\left(M_{k}^{\bigotimes d_{k}}\right)$ for $k=1, \ldots, m$, and let $Q$ be a nonzero quotient of the module Ind $\left(\underset{k=1}{\bigotimes} N_{k}\right)$. By (ii) and Lemma 1.5 (2), we have an injective homomorphism

$$
\bigotimes_{k=1}^{\boxplus} N_{k} \hookrightarrow \operatorname{Res}_{d_{1} \beta_{1}, \ldots, d_{m} \beta_{m}} Q .
$$

Hence, by (iii), i occurs in $\operatorname{ch} Q$ with multiplicity $\xi_{1} \xi_{2} \cdots \xi_{m}$. Since $Q$ is arbitrary, we obtain that hd Ind $\left(\underset{k=1}{\bigotimes} N_{k}\right)$ is irreducible.

Now it follows from (i) that

$$
\operatorname{lnd}\left(\underset{k=1}{\mathrm{~m}} N_{k}\right) \simeq \operatorname{lnd}\left(N_{m} \otimes N_{m-1} \otimes \cdots \otimes N_{1}\right) .
$$

Let $L$ be a nonzero submodule of Ind $\left(\underset{k=1}{\bigotimes} N_{k}\right)$. By (ii) and Lemma 1.5, there exists a surjective homomorphism

$$
\operatorname{Res} L \rightarrow \underset{k=1}{m} N_{k}
$$

which implies that $\mathbf{i}$ occurs in $\operatorname{ch} L$ with multiplicity $\xi_{1} \xi_{2} \cdots \xi_{m}$ by (iii). Therefore, we conclude Ind $\left(\underset{k=1}{\bigotimes} N_{k}\right)=\operatorname{lnd}\left(\underset{k=1}{\bigotimes} M_{k}^{\bigotimes d_{k}}\right)$ is irreducible.

Lemma 1.8. Let $\emptyset=I_{(0)} \subsetneq I_{(1)} \subsetneq I_{(2)} \subsetneq \cdots \subsetneq I_{(m)} \subset I$, and assume $\beta_{k} \in \sum_{i \in I_{(k)}} \mathbb{Z}_{\geq 0} \alpha_{i}$ for $k=1, \ldots, m$. Let $M_{k} \in R\left(\beta_{k}\right)$-fmod $(1 \leq k \leq m)$ be such that

(i) $\varepsilon_{i}\left(M_{k}\right)=0$ for $i \in I_{(k-1)}$,

(ii) $\mathrm{hd} M_{k}$ is irreducible, and

(iii) hd $M_{k}$ occurs with multiplicity one as a composition factor of $M_{k}$.

Then

(1) hd Ind $\left(\underset{k=1}{\otimes} M_{k}\right)$ is irreducible,

(2) hd Ind $\left(\underset{k=1}{\underset{m}{\bigotimes}} M_{k}\right)$ occurs with multiplicity one as a composition factor of $\operatorname{Ind}\left(\underset{k=1}{\otimes} M_{k}\right)$,

(3) if $\tilde{f}_{\mathbf{i}_{k}} \mathbf{1} \simeq$ hd $M_{k}$ for some $\mathbf{i}_{k} \in I^{\beta_{k}}$, then $\tilde{f}_{\mathbf{i}_{1}} \tilde{f}_{\mathbf{i}_{2}} \cdots \tilde{f}_{\mathbf{i}_{m}} \mathbf{1} \simeq$ hd Ind $\left(\underset{k=1}{\bigotimes} M_{k}\right)$.

Proof. We may assume that $M_{k} \neq \mathbf{1}$ for $k=1, \ldots, m$. Let $d_{k}=\left|\beta_{k}\right|$ for $k=1, \ldots, m$, and set $d=d_{1}+\cdots+d_{m}$. For $w \in S_{d}$ and a reduced expression $w=\sigma_{i_{1}} \cdots \sigma_{i_{s}}$, let

$$
\tau_{w}=\tau_{i_{1}} \cdots \tau_{i_{s}}
$$


For $k=1, \ldots, m$, take $\mathbf{i}_{k}=\left(i_{k, 1}, \ldots, i_{k, d_{k}}\right) \in I^{\beta_{k}}$ such that $e\left(\mathbf{i}_{k}\right) M_{k} \neq 0$. It follows from (i) that $i_{k, 1} \in I_{(k)} \backslash I_{(k-1)}$. Since $M_{k} \in R\left(\beta_{k}\right)$-fmod, we have

$$
e\left(\beta_{1}, \ldots, \beta_{m}\right) \tau_{w} e\left(\mathbf{i}_{1} * \cdots * \mathbf{i}_{m}\right) \neq 0 \quad \text { if and only if } w=\text { id }
$$

for $w \in S_{d} / S_{d_{1}} \times \cdots \times S_{d_{m}}$. Therefore,

$$
\operatorname{Res}_{\beta_{1}, \ldots, \beta_{m}}\left(\operatorname{lnd}\left(\underset{k=1}{\bigotimes} M_{k}\right)\right) \simeq \underset{k=1}{\bigotimes} M_{k} .
$$

Let $L=\bigotimes_{k=1}^{m} \mathrm{hd} M_{k}$. It follows from (ii) and (iii) that $[L]$ occurs with multiplicity one in $\left[\operatorname{Res}_{\beta_{1}, \ldots, \beta_{m}}\left(\operatorname{Ind}\left(\bigotimes_{k=1}^{m} M_{k}\right)\right)\right]$ in the Grothendieck group $G_{0}\left(R\left(\beta_{1}\right) \otimes \cdots \otimes R\left(\beta_{m}\right)\right)$. Let $Q$ be a nonzero quotient of Ind $\left(\underset{k=1}{\otimes} M_{k}\right)$. By Lemma 1.5, we have a nontrivial homomorphism

$$
\underset{k=1}{\bigotimes} M_{k} \rightarrow \operatorname{Res}_{\beta_{1}, \ldots, \beta_{m}} Q
$$

which implies that $[L]$ occurs in $\left[\operatorname{Res}_{\beta_{1}, \ldots, \beta_{m}} Q\right]$ with multiplicity one. Since $Q$ is arbitrary, we obtain assertions (1) and (2).

Now assume that $N=$ hd Ind $\left(\underset{k=1}{\otimes} M_{k}\right)$, and let $N_{k}=\mathrm{hd} M_{k}$ for $k=1, \ldots, m$. Then there is a surjective homomorphism

$$
\operatorname{Ind}\left(\underset{k=1}{\otimes} M_{k}\right) \rightarrow \operatorname{lnd}\left(\underset{k=1}{\bigotimes} N_{k}\right),
$$

which implies that $N=$ hd Ind $\left(\underset{k=1}{\otimes} N_{k}\right)$. We will use induction on $|w t(N)|$. If $|\operatorname{wt}(N)|=0$, there is nothing to prove, so suppose that $|\operatorname{wt}(N)|>0$. Since $N_{1}$ is nontrivial, we can take $i \in I_{(1)}$ such that $\varepsilon_{i}\left(N_{1}\right) \neq 0$. Let $\varepsilon=\varepsilon_{i}\left(N_{1}\right)$. Then, by (i) and Lemma 1.5, we have

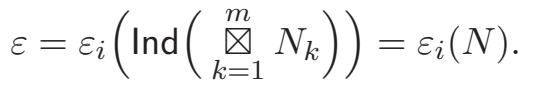

Since $e_{i}$ is exact, there is a surjective homomorphism

$$
e_{i}^{\varepsilon}\left(\operatorname{lnd}\left({\underset{\aleph=1}{m}}_{k=1}^{m} N_{k}\right)\right) \rightarrow e_{i}^{\varepsilon} N .
$$

Then, by [15, Lem. 3.9], we know that $\left[e_{i}^{\varepsilon} N\right]=q_{i}^{-\varepsilon+1}[\varepsilon]_{q_{i}} !\left[\tilde{e}_{i}^{\varepsilon} N\right]$ and

$$
\begin{aligned}
{\left[e_{i}^{\varepsilon}\left(\operatorname{lnd}\left(\underset{k=1}{\otimes} N_{k}\right)\right)\right] } & =\left[\operatorname{lnd}\left(\left(e_{i}^{\varepsilon} N_{1}\right) \otimes N_{2} \otimes \cdots \otimes N_{m}\right)\right] \\
& =q_{i}^{-\varepsilon+1}[\varepsilon]_{q_{i}} !\left[\operatorname{lnd}\left(\left(\tilde{e}_{i}^{\varepsilon} N_{1}\right) \otimes N_{2} \otimes \cdots \otimes N_{m}\right)\right]
\end{aligned}
$$

at the level of the Grothendieck group $G_{0}\left(R\left(\beta_{1}+\cdots+\beta_{m}-\varepsilon \alpha_{i}\right)\right)$. Since hd Ind $\left(\left(\tilde{e}_{i}^{\varepsilon} N_{1}\right) \otimes\right.$ $\left.N_{2} \otimes \cdots \otimes N_{m}\right)$ is irreducible by (1), we obtain

$$
\operatorname{hd} \operatorname{lnd}\left(\tilde{e}_{i}^{\varepsilon} N_{1} \otimes \cdots \otimes N_{m}\right) \simeq \tilde{e}_{i}^{\varepsilon} N .
$$


Therefore, assertion (3) follows from a standard induction argument.

Let $n=|I|$ be the rank of $U_{q}(\mathfrak{g})$, and let $I_{(k)} \subset I(k=1, \ldots, n)$ be subsets of $I=I_{(n+1)}$ such that $I_{(k)} \subset I_{(k+1)}$ and $\left|I_{(k)}\right|=k$ for all $k$. We denote by $U_{q}\left(\mathfrak{g}_{k}\right)$ the subalgebra of $U_{q}(\mathfrak{g})$ generated by $e_{i}, f_{i}\left(i \in I_{(k)}\right)$ and $q^{h}\left(h \in \mathrm{P}^{\vee}\right)$. Let $\mathfrak{B}_{k}$ be the crystal obtained from $B(\infty)$ by forgetting the $i$-arrows for $i \notin I_{(k)}$. Then $\mathfrak{B}_{k}$ can be viewed as a $U_{q}\left(\mathfrak{g}_{k}\right)$-crystal.

Lemma 1.9. For each $k=1, \ldots, n$, every connected component of $\mathfrak{B}_{k}$ has a unique highest weight vector.

Proof. Let $\mathcal{C}$ be a connected component of $\mathfrak{B}_{k}$. For $\lambda \in \mathrm{P}^{+}$, let $\iota_{\lambda}: B(\lambda) \rightarrow B(\infty) \otimes T_{\lambda} \otimes C$ be the embedding in (1.1) and let

$$
\widetilde{\mathcal{C}}=\left\{v \otimes t_{\lambda} \otimes c \mid v \in \mathcal{C}\right\} \subset B(\infty) \otimes T_{\lambda} \otimes C .
$$

By taking $\lambda \gg 0$, we can assume that $\widetilde{\mathcal{C}} \cap \operatorname{im}\left(\iota_{\lambda}\right) \neq \emptyset$. Since $\mathcal{C}_{\lambda}:=\iota_{\lambda}^{-1}(\widetilde{\mathcal{C}})$ is a nontrivial highest weight subcrystal of the crystal obtained from $B(\lambda)$ by forgetting the $i$-arrows with $i \notin I_{(k)}$, there is an element $u_{\mathcal{C}} \in \mathcal{C}$ such that $\iota_{\lambda}^{-1}\left(u_{\mathcal{C}} \otimes t_{\lambda} \otimes c\right)$ is the highest weight vector of the subcrystal $\mathcal{C}_{\lambda}$. Note that $u_{\mathcal{C}}$ does not depend on the choice of $\lambda$ if $\widetilde{\mathcal{C}} \cap \operatorname{im}\left(\iota_{\lambda}\right) \neq \emptyset$. By construction, the element $u_{\mathcal{C}}$ is a unique highest weight vector of $\mathcal{C}$.

Take $v \in B(\infty)$. Let $u_{0}=v$ and let $u_{k}$ be the highest weight vector of the connected component $\mathcal{C}_{k}$ of $\mathfrak{B}_{k}$ containing $v$ for $k=1, \ldots, n$. By construction, there is a chain of injective maps

$$
\mathcal{C}_{1} \hookrightarrow \mathcal{C}_{2} \hookrightarrow \cdots \hookrightarrow \mathcal{C}_{n-1} \hookrightarrow B(\infty)
$$

For $k=1, \ldots, n$, let $\mathbf{i}_{k}$ be a sequence of $I$ such that $u_{k-1}=\tilde{f}_{\mathbf{i}_{k}} u_{k}$. Note that $v=\tilde{f}_{\mathbf{i}_{1}} \tilde{f}_{\mathbf{i}_{1}} \cdots \tilde{f}_{\mathbf{i}_{n}} 1$. Let

$$
\mathcal{N}_{k}(v)=\tilde{f}_{\mathbf{i}_{k}} \mathbf{1} \in \mathbb{B}(\infty) .
$$

Hence, for each $v \in B(\infty)$, we have the corresponding $n$-tuple $\left(\mathcal{N}_{1}(v), \mathcal{N}_{2}(v), \ldots, \mathcal{N}_{n}(v)\right)$ of modules in $\mathbb{B}(\infty)$.

\section{Proposition 1.10.}

(1) For $v \in B(\infty)$, let $\mathcal{N}_{k}(v)$ be the irreducible module defined by (1.4). Then $\operatorname{hd}$ Ind $\left(\underset{k=1}{\otimes} \mathcal{N}_{k}(v)\right)$ is irreducible.

(2) The map $\Phi: B(\infty) \longrightarrow \mathbb{B}(\infty)$ defined by

$$
\Phi(v)=\operatorname{hd} \operatorname{lnd}\left(\bigotimes_{k=1}^{n} \mathcal{N}_{k}(v)\right) \quad \text { for } v \in B(\infty)
$$

is a crystal isomorphism. 
Proof. Since it is obvious that $\Phi(1)=\mathbf{1}$, we assume $1 \neq v \in B(\infty)$ and let $\mathcal{N}_{k}(v)=\tilde{f}_{\mathbf{i}_{k}} \mathbf{1}$ be the corresponding irreducible module given in (1.4) for $k=1, \cdots, n$. By construction, we have

$$
v=\tilde{f}_{\mathbf{i}_{1}} \tilde{f}_{\mathbf{i}_{1}} \cdots \tilde{f}_{\mathbf{i}_{n}} 1 \quad \text { and } \quad \varepsilon_{i}\left(\mathcal{N}_{k}(v)\right)=0 \quad \text { for all } i \in I_{(k-1)} .
$$

Therefore, it follows from Lemma 1.8 that hd Ind $\left(\underset{k=1}{\bigotimes} \mathcal{N}_{k}(v)\right)$ is irreducible and

$$
\Phi\left(\tilde{f}_{\mathbf{i}_{1}}{\tilde{\mathbf{i}_{2}}}_{\mathbf{i}_{2}} \cdots \tilde{f}_{\mathbf{i}_{n}} 1\right)=\operatorname{hd} \operatorname{lnd}\left(\underset{k=1}{\bigotimes} \mathcal{N}_{k}(v)\right)=\tilde{f}_{\mathbf{i}_{1}}{\tilde{\mathbf{i}_{2}}}_{\mathbf{i}_{2}} \cdots \tilde{f}_{\mathbf{i}_{n}} \Phi(1)
$$

\section{Description of $\mathcal{N}_{k}(v)$ for Finite Type Via Adapted Strings}

In this section, we give an explicit description of the modules $\mathcal{N}_{k}(v)$ for finite type. To that end, we choose a particular reduced expression of the longest element $w_{0}$ of the Weyl group $W$. Using the notion of adapted strings for crystals given in 24] with respect to these specially chosen expressions, we describe $\mathcal{N}_{k}(v)$ explicitly in terms of Kashiwara operators $\tilde{f}_{i}$ (Proposition 2.3).

Throughout the section, we assume that the Cartan datum $\left(\mathfrak{A}, P, \Pi, \mathrm{P}^{\vee}, \Pi^{\vee}\right)$ is of finite type. For a sequence $\mathbf{m}=\left(m_{1}, \ldots, m_{k}\right)$ of elements in $I$, we write $r_{\mathbf{m}}=r_{m_{1}} r_{m_{2}} \cdots r_{m_{k}}$ for the corresponding element in the Weyl group. Using this convention, we fix a particular reduced expression for the longest element $w_{0}$ of $W$

$$
w_{0}=r_{\mathbf{s}_{1}} r_{\mathbf{s}_{2}} \cdots r_{\mathbf{s}_{n}}=r_{s_{1}} r_{s_{2}} \cdots r_{s_{\ell}}
$$

where the sequences $\mathbf{s}_{k}$ are displayed in Table 1 below. Let $l_{k}$ be the length of the sequence $\mathbf{s}_{k}$ in (2.1) for $k=1, \ldots, n$. Then $r_{\mathbf{s}_{1}}=r_{s_{1}} \cdots r_{s_{l_{1}}}, r_{\mathbf{s}_{2}}=r_{s_{l_{1}+1}} \cdots r_{s_{l_{1}+l_{2}}}$ and so forth. Note that $\ell$ is the length of the reduced expression.

Definition 2.1. Let $v \in B(\infty)$ (resp. $v \in B(\lambda)$ ). An $\ell$-tuple $\mathbf{a}(v)=\left(a_{1}, \ldots, a_{\ell}\right) \in\left(\mathbb{Z}_{\geq 0}\right)^{\ell}$ is called the adapted string of $v$ with respect to the expression $r_{s_{1}} \cdots r_{s_{\ell}}$ if

$$
a_{1}=\varepsilon_{s_{1}}(v), \quad a_{2}=\varepsilon_{s_{2}}\left(\tilde{e}_{s_{1}}^{a_{1}} v\right), \ldots, a_{\ell}=\varepsilon_{s_{\ell}}\left(\tilde{e}_{s_{\ell-1}}^{a_{\ell-1}} \ldots \tilde{e}_{s_{1}}^{a_{1}} v\right) .
$$

We denote by $\mathcal{S}=\{\mathbf{a}(v) \mid v \in B(\infty)\}$ (resp. $\mathcal{S}^{\lambda}=\{\mathbf{a}(v) \mid v \in B(\lambda)\}$ ) the set of all adapted strings of elements in $B(\infty)$ (resp. $B(\lambda)$ ).

Observe that if $\iota_{\lambda}(v)=v^{\prime} \otimes t_{\lambda} \otimes c$ for some $v \in B(\lambda)$ and $v^{\prime} \in B(\infty)$, then $\mathbf{a}(v)=\mathbf{a}\left(v^{\prime}\right)$. It follows from [17, 23] that $\mathcal{S}$ and $\mathcal{S}^{\lambda}$ are in one-to-one correspondence with the crystals $B(\infty)$ 
TABle 1.

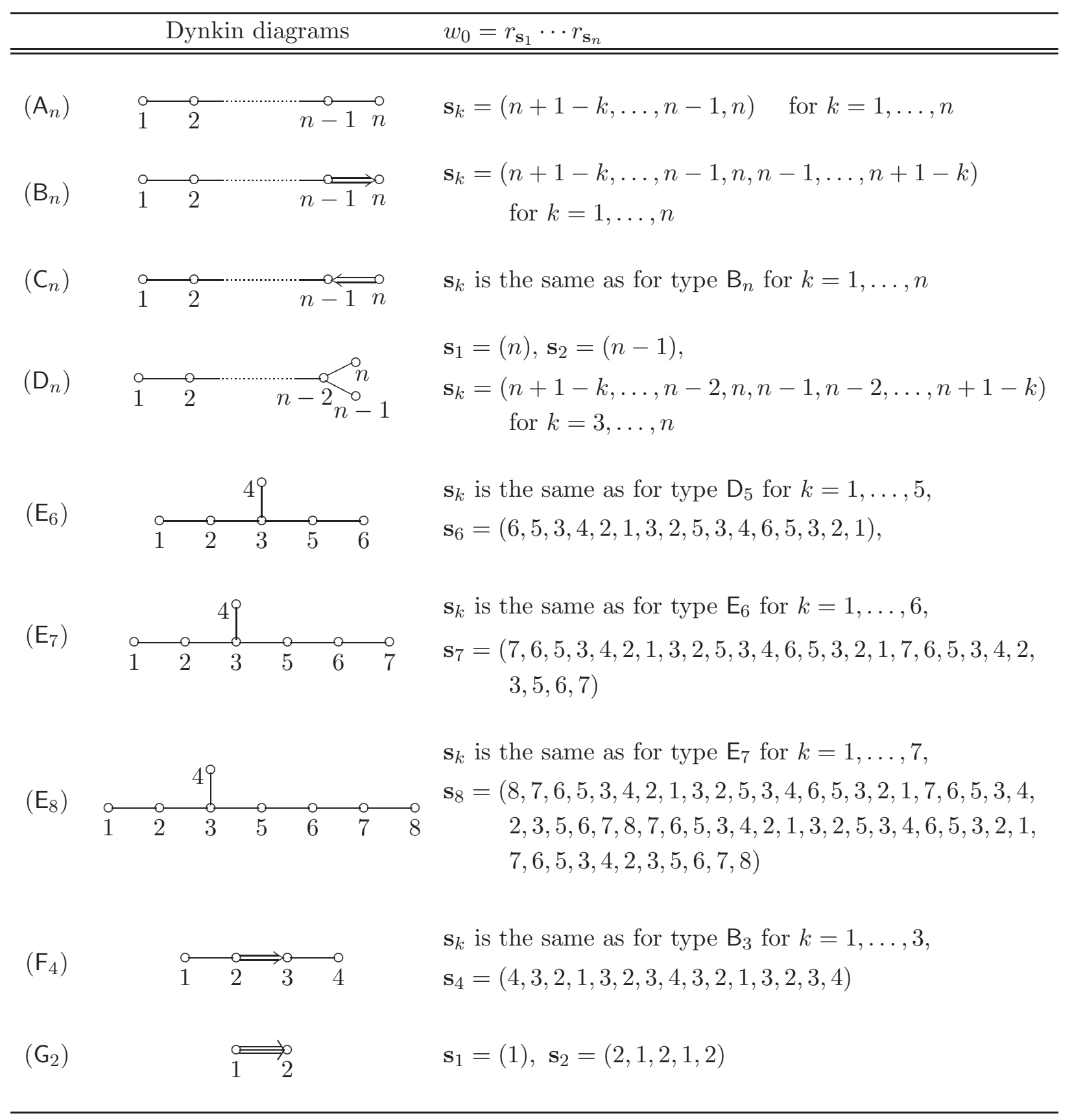

and $B(\lambda)$, respectively, by the maps

$$
\begin{array}{ccc}
\mathcal{S} \stackrel{1-1}{\longrightarrow} B(\infty) & \text { given by } & \mathbf{a}=\left(a_{1}, \ldots, a_{\ell}\right) \mapsto \tilde{f}_{s_{1}}^{a_{1}} \cdots \tilde{f}_{s_{\ell}}^{a_{\ell}} 1, \\
\mathcal{S}^{\lambda} \stackrel{1-1}{\longrightarrow} B(\lambda) & \text { given by } & \mathbf{a}=\left(a_{1}, \ldots, a_{\ell}\right) \mapsto \tilde{f}_{s_{1}}^{a_{1}} \cdots \tilde{f}_{s_{\ell}}^{a_{\ell}} b_{\lambda} .
\end{array}
$$


For $\mathbf{a}=\left(a_{1}, \ldots, a_{\ell}\right) \in\left(\mathbb{Z}_{\geq 0}\right)^{\ell}$, set

$$
\mathrm{a}_{k, j}=a_{l_{1}+\cdots+l_{k-1}+j} \quad \text { and } \quad \mathbf{a}_{k}=\left(\mathrm{a}_{k, 1}, \mathrm{a}_{k, 2}, \ldots, \mathrm{a}_{k, l_{k}}\right),
$$

where $l_{k}$ is, as above, the length of $\mathbf{s}_{k}$. Then $\mathbf{a}=\mathbf{a}_{\mathbf{1}} * \cdots * \mathbf{a}_{\mathbf{n}}$.

In particular, for $v \in B(\infty)$, we have $\mathbf{a}(v)=\mathbf{a}(v)_{1} * \cdots * \mathbf{a}(v)_{n}$ and

$$
v=\tilde{f}_{\mathbf{s}_{1}}^{\mathbf{a}(v)_{1}} \tilde{f}_{\mathbf{s}_{2}}^{\mathbf{a}(v)_{2}} \cdots \tilde{f}_{\mathbf{s}_{n}}^{\mathbf{a}(v)_{n}} 1
$$

Proposition $2.2([24])$. Let $\left(\mathfrak{A}, \mathrm{P}, \Pi, \mathrm{P}^{\vee}, \Pi^{\vee}\right)$ be a Cartan datum of finite type. Then $\mathcal{S}$ has the following description:

$$
\begin{array}{cc}
\left(\mathrm{A}_{n}\right): & \left\{\mathbf{a} \in\left(\mathbb{Z}_{\geq 0}\right)^{\ell} \mid \mathrm{a}_{i, 1} \geq \mathrm{a}_{i, 2} \geq \cdots \geq \mathrm{a}_{i, i}(1 \leq i \leq n)\right\} \\
\left(\mathrm{B}_{n}\right): & \left\{\mathbf{a} \in\left(\mathbb{Z}_{\geq 0}\right)^{\ell} \mid 2 \mathrm{a}_{i, 1} \geq \cdots \geq 2 \mathrm{a}_{i, i-1} \geq \mathrm{a}_{i, i} \geq 2 \mathrm{a}_{i, i+1} \geq \cdots \geq 2 \mathrm{a}_{i, 2 i-1}(1 \leq i \leq n)\right\} \\
\left(\mathrm{C}_{n}\right): & \left\{\mathbf{a} \in\left(\mathbb{Z}_{\geq 0}\right)^{\ell} \mid \mathrm{a}_{i, 1} \geq \cdots \geq \mathrm{a}_{i, i-1} \geq \mathrm{a}_{i, i} \geq \mathrm{a}_{i, i+1} \geq \cdots \geq \mathrm{a}_{i, 2 i-1}(1 \leq i \leq n)\right\} \\
\left(\mathrm{D}_{n}\right): & \left\{\mathbf{a} \in\left(\mathbb{Z}_{\geq 0}\right)^{\ell} \mid \mathrm{a}_{i, 1} \geq \cdots \geq \mathrm{a}_{i, i-1}, \mathrm{a}_{i, i} \geq \mathrm{a}_{i, i+1} \geq \cdots \geq \mathrm{a}_{i, 2 i-2}(3 \leq i \leq n)\right\} \\
\left(\mathrm{E}_{6}\right): & \left\{\mathbf{a} \in\left(\mathbb{Z}_{\geq 0}\right)^{\ell} \mid \mathrm{a}_{6,1} \geq \mathrm{a}_{6,2} \geq \mathrm{a}_{6,3} \geq \mathrm{a}_{6,4}, \mathrm{a}_{6,5} \geq \mathrm{a}_{6,7} \geq \mathrm{a}_{6,8}, \mathrm{a}_{6,9} \geq \mathrm{a}_{6,10} \geq \mathrm{a}_{6,11}, \mathrm{a}_{6,13}\right. \\
\geq \mathrm{a}_{6,14} \geq \mathrm{a}_{6,15} \geq \mathrm{a}_{6,16} ; \mathrm{a}_{6,5} \geq \mathrm{a}_{6,6} \geq \mathrm{a}_{6,8} ; \mathrm{a}_{6,9} \geq \mathrm{a}_{6,12} \geq \mathrm{a}_{6,13} ; \\
\left.\mathrm{a}_{i, j}{ }^{\prime} \text { satisfy the inequalities for } \mathrm{D}_{5} \text { for } i=1, \ldots, 5\right\} \\
\left(\mathrm{E}_{7}\right): \quad\left\{\mathbf{a} \in\left(\mathbb{Z}_{\geq 0}\right)^{\ell} \mid \mathrm{a}_{7,1} \geq \mathrm{a}_{7,2} \geq \mathrm{a}_{7,3} \geq \mathrm{a}_{7,4} \geq \mathrm{a}_{7,5}, \mathrm{a}_{7,6} \geq \mathrm{a}_{7,8} \geq \mathrm{a}_{7,9}, \mathrm{a}_{7,10}\right. \\
\geq \mathrm{a}_{7,11} \geq \mathrm{a}_{7,12}, \mathrm{a}_{7,14} \geq \mathrm{a}_{7,15} \geq \mathrm{a}_{7,16}, \mathrm{a}_{7,20} \geq \mathrm{a}_{7,21} \geq \mathrm{a}_{7,22}, \mathrm{a}_{7,23} \\
\geq \mathrm{a}_{7,24} \geq \mathrm{a}_{7,25} \geq \mathrm{a}_{7,26} \geq \mathrm{a}_{7,27} ; \mathrm{a}_{7,6} \geq \mathrm{a}_{7,7} \geq \mathrm{a}_{7,8} ; \\
\mathrm{a}_{7,10} \geq \mathrm{a}_{7,13} \geq \mathrm{a}_{7,14}, \mathrm{a}_{7,18} \geq \mathrm{a}_{7,19} \geq \mathrm{a}_{7,20} ; \mathrm{a}_{7,16} \geq \mathrm{a}_{7,17} \geq \mathrm{a}_{7,23} ; \\
\left.\mathrm{a}_{i, j}{ }^{\prime} \text { s satisfy the inequalities for } \mathrm{E}_{6} \text { for } i=1, \ldots, 6\right\}
\end{array}
$$

$\left(\mathrm{E}_{8}\right): \quad\left\{\mathbf{a} \in\left(\mathbb{Z}_{\geq 0}\right)^{\ell} \mid\left(\mathrm{a}_{8,2}, \ldots, \mathrm{a}_{8,28}\right)\right.$ and $\left(\mathrm{a}_{8,30}, \ldots, \mathrm{a}_{8,56}\right)$ satisfy the inequalities of $\mathbf{a}_{7}$ for $\mathrm{E}_{7}$;

$$
\begin{aligned}
& a_{8,1} \geq a_{8,2} ; a_{8,29} \geq a_{8,30} ; a_{8,56} \geq a_{8,57} ; \\
& a_{8,19} \geq \max \left\{a_{8,30}, a_{8,29}-a_{8,28}\right\} ; \min \left\{a_{8,23}, a_{8,25}+a_{8,33}-a_{8,34}\right\} \geq a_{8,35} ; \\
& a_{8,20} \geq \max \left\{a_{8,31}, a_{8,28}+a_{8,30}-a_{8,27}\right\} ; \min \left\{a_{8,25}, a_{8,26}+a_{8,32}-a_{8,33}\right\} \geq a_{8,37} ; \\
& a_{8,21} \geq \max \left\{a_{8,32}, a_{8,27}+a_{8,31}-a_{8,26}\right\} ; \min \left\{a_{8,26}, a_{8,27}+a_{8,31}-a_{8,32}\right\} \geq a_{8,39} ; \\
& a_{8,22} \geq \max \left\{a_{8,33}, a_{8,26}+a_{8,32}-a_{8,25}\right\} ; \min \left\{a_{8,27}, a_{8,28}+a_{8,30}-a_{8,31}\right\} \geq a_{8,42} ; \\
& a_{8,24} \geq \max \left\{a_{8,34}, a_{8,25}+a_{8,33}-a_{8,23}\right\} ; \min \left\{a_{8,28}, a_{8,29}-a_{8,30}\right\} \geq a_{8,47} ; \\
& \left.a_{i, j}{ }^{\prime} \text { satisfy the inequalities for } \mathrm{E}_{7} \text { for } i=1, \ldots, 7\right\}
\end{aligned}
$$




$$
\begin{aligned}
\left(F_{4}\right):\left\{\mathbf{a} \in\left(\mathbb{Z}_{\geq 0}\right)^{\ell} \mid\right. & \mathrm{a}_{4,1} \geq \mathrm{a}_{4,2} \geq \mathrm{a}_{4,3} \geq \mathrm{a}_{4,4}, \mathrm{a}_{4,5} \geq \mathrm{a}_{4,6} \geq \mathrm{a}_{4,7} ; \\
& \mathrm{a}_{4,9} \geq \mathrm{a}_{4,10} \geq \mathrm{a}_{4,11}, \mathrm{a}_{4,12} \geq \mathrm{a}_{4,13} \geq \mathrm{a}_{4,14} \geq \mathrm{a}_{4,15} ; \\
& \mathrm{a}_{4,5} \geq \mathrm{a}_{4,9} ; \mathrm{a}_{4,7} \geq \mathrm{a}_{4,12} ; \mathrm{a}_{4,5}+\mathrm{a}_{4,7} \geq \mathrm{a}_{4,8} \geq \mathrm{a}_{4,9}+\mathrm{a}_{4,12} ; \\
& 2 \mathrm{a}_{4,6} \geq \mathrm{a}_{4,7}+\mathrm{a}_{4,9} \geq 2 \mathrm{a}_{4,10} ; \\
& \left.\mathrm{a}_{i, j} \text { 's satisfy the inequalities for } \mathrm{B}_{3} \text { for } i=1,2,3\right\} \\
\left(\mathrm{G}_{2}\right):\left\{\mathbf{a} \in\left(\mathbb{Z}_{\geq 0}\right)^{\ell} \mid\right. & \left.6 \mathrm{a}_{2,1} \geq 2 \mathrm{a}_{2,2} \geq 3 \mathrm{a}_{2,3} \geq 2 \mathrm{a}_{2,4} \geq 6 \mathrm{a}_{2,5}\right\} .
\end{aligned}
$$

We now give a description of $\mathcal{N}_{k}(v)$ using Proposition 2.2.

Proposition 2.3. Let $\left(\mathfrak{A}, \mathrm{P}, \Pi, \mathrm{P}^{\vee}, \Pi^{\vee}\right)$ be a Cartan datum of finite type. For $v \in B(\infty)$ and $k=1, \ldots, n$, the module $\mathcal{N}_{k}(v)$ defined by (1.4) is given by

$$
\mathcal{N}_{k}(v)=\tilde{f}_{\mathbf{s}_{k}}^{\mathbf{a}(v)_{k}} \mathbf{1},
$$

where $\mathbf{a}(v)$ is the adapted string of $v$ with respect to the expression $w_{0}=r_{\mathbf{s}_{1}} \cdots r_{\mathbf{s}_{n}}$ in Table 1 .

Proof. Let $I_{(k)}=\{n+1-k, \ldots, n-1, n\}$ for $k=1, \ldots, n$ and set $I_{(0)}=\emptyset$. Let $\mathfrak{B}_{k}$ denote the crystal obtained from $B(\infty)$ by forgetting the $i$-arrows with $i \notin I_{(k)}$. Take $v \in B(\infty)$ and let $\mathbf{a}=\mathbf{a}(v)=\mathbf{a}_{1} * \cdots * \mathbf{a}_{n}$ be the adapted string of $v$ with respect to the expression $w_{0}=r_{\mathbf{s}_{1}} \cdots r_{\mathbf{s}_{n}}$ in Table 1, For $k=1, \ldots, n$, let $\mathbf{0}_{k}=(\underbrace{0, \ldots, 0}_{l_{k}})$ where $l_{k}$ is the length of $\mathbf{s}_{k}$ as before. Then, by Proposition 2.2 ,

$$
\mathbf{b}_{k}:=\mathbf{0}_{1} * \ldots * \mathbf{0}_{k} * \mathbf{a}_{k+1} * \ldots * \mathbf{a}_{n-1} * \mathbf{a}_{n}
$$

is contained in $\mathcal{S}$. Let $u_{k}=\tilde{f}_{\mathbf{s}_{k+1}}^{\mathbf{a}_{k+1}} \cdots \tilde{f}_{\mathbf{s}_{n}}^{\mathbf{a}_{n}} 1$ for $k=0, \ldots, n-1$ and let $u_{n}=1$. Since $\mathbf{b}_{k}$ is the adapted string of $u_{k}$, by the definition of adapted strings we have

$$
v=\tilde{f}_{\mathbf{s}_{1}}^{\mathbf{a}_{1}} \cdots \tilde{f}_{\mathbf{s}_{k}}^{\mathbf{a}_{k}} u_{k}, \quad \varepsilon_{i}\left(u_{k}\right)=0 \text { for } i \in I_{(k)},
$$

which implies that $u_{k}$ is the highest weight vector of the connected component $\mathcal{C}_{k}$ of $\mathfrak{B}_{k}$ containing $v$. Therefore, $\mathcal{N}_{k}(v)=\tilde{f}_{\mathbf{s}_{k}}^{\mathbf{a}_{k}} \mathbf{1}$ for $k=1, \ldots, n$.

\section{Explicit Construction of Irreducible Modules for Finite Classical Type}

In Sections 3 and 4 , we assume that $\mathfrak{A}$ is of finite classical type. We maintain the notation from Sections 1 and 2. Our aim in this section is to present an explicit construction of irreducible $R$-modules (resp. $R^{\lambda}$-modules) for finite classical type using a single induction step. Let $\mathbf{B}$ be the crystal of the irreducible highest weight $U_{q}(\mathfrak{g})$-module $V\left(\Lambda_{n}\right)$ if $\mathfrak{A}$ is of type $\mathrm{A}_{n}$ and of $V\left(\Lambda_{1}\right)$ if $\mathfrak{A}$ is of type $\mathrm{B}_{n}, \mathrm{C}_{n}, \mathrm{D}_{n}$. We define the 1 or 2 -dimensional irreducible 
$R$-module $\Delta_{(a, b)}$ for $a, b \in \mathbf{B}$ with $a \succ b$ by using the structure of $\mathbf{B}$. Combining Proposition 1.10 with some facts about the modules $\Delta_{(a, b)}$ and the descriptions of the adapted strings, we define the outer tensor product $\Delta(\mathbf{a}(v)):=\Delta(\mathbf{a}(v) ; 1) \nabla \cdots \nabla \Delta(\mathbf{a}(v) ; n)$ of $\Delta_{(a, b)}$ 's for $v \in B(\infty)$ (resp. $v \in B(\lambda)$ ). For $v \in B(\lambda)$, the number $\eta(v)$ of $\Delta_{(a, b)}$ 's in $\Delta(\mathbf{a}(v)$ ) has an upper bound; i.e., $\eta(v) \leq n \lambda(h)$, where $h$ is as in Lemma 3.4 below. Then, we construct a map $\Psi: B(\infty) \rightarrow \mathbb{B}(\infty)$ (resp. $\Psi^{\lambda}: B(\lambda) \rightarrow \mathbb{B}(\lambda)$ ) by taking the head of Ind $\Delta(\mathbf{a}(v)$ ) (Theorem 3.2). The proof that this map is indeed a crystal isomorphism, hence is compatible with the Kashiwara operators, will be provided in the next section.

We first give a detailed description of $\mathcal{S}$ and $\mathcal{S}^{\lambda}$ for finite classical type with respect to the expression of $w_{0}=r_{\mathbf{s}_{1}} \cdots r_{\mathbf{s}_{n}}$ in Table 1. Let $\triangle_{\mathrm{A}_{n}}$ be the triangle consisting of right justified rows of boxes with 1 box in the first (bottom) row, 2 boxes in the second row, ..., and $n$ boxes in the top row. Let $\triangle_{\mathrm{B}_{n}}$ (resp. $\triangle_{\mathrm{D}_{n}}$ ) be the triangle consisting of centered rows of boxes having 1 box (resp. 2 boxes) in the first row, 3 boxes (resp. 4 boxes) in the second row, $\ldots$, and $(2 n-1)$ boxes (resp. $(2 n-2)$ boxes) in the top row. Set $\triangle_{\mathrm{C}_{n}}=\triangle_{\mathrm{B}_{n}}$. When it is not necessary to specify the type, we omit the subscript and simply write $\triangle$. For $\mathbf{a} \in \mathcal{S}$, let $\triangle(\mathbf{a})$ denote the filling of the triangle $\triangle$ with entries of a from left to right in each row, and from bottom to top. Let $t_{i j}$ be the $j$ th entry of the $i$ th row in $\triangle(\mathbf{a})$ and let $\triangle(\mathbf{a})=\left\{t_{i j}\right\}_{1 \leq i \leq n^{\prime}, p_{i} \leq j \leq p_{i}^{\prime}}$, where

$$
\begin{aligned}
n^{\prime} & = \begin{cases}n & \left(\mathrm{~A}_{n}, \mathrm{~B}_{n}, \mathrm{C}_{n}\right) \\
n-1 & \left(\mathrm{D}_{n}\right)\end{cases} \\
p_{i} & = \begin{cases}n+1-i & \left(\mathrm{~A}_{n}, \mathrm{~B}_{n}, \mathrm{C}_{n}\right) \\
n-i & \left(\mathrm{D}_{n}\right)\end{cases} \\
p_{i}^{\prime} & = \begin{cases}n & \left(\mathrm{~A}_{n}\right), \\
n-1+i & \left(\mathrm{~B}_{n}, \mathrm{C}_{n}, \mathrm{D}_{n}\right)\end{cases}
\end{aligned}
$$

Set $t_{i j}=0$ except when $1 \leq i \leq n^{\prime}, p_{i} \leq j \leq p_{i}^{\prime}$. For example, if $\mathbf{a}=(2,3,1,0,9,8,4,2,1) \in \mathcal{S}$ for type $\mathrm{B}_{3}$ and $\mathbf{a}^{\prime}=(5,2,7,4,3,1,9,6,4,5,3,2) \in \mathcal{S}$ for type $\mathrm{D}_{4}$, then $t_{1,3}=2$ in $\triangle(\mathbf{a})$, $t_{3,4}=5$ in $\triangle\left(\mathbf{a}^{\prime}\right)$ and

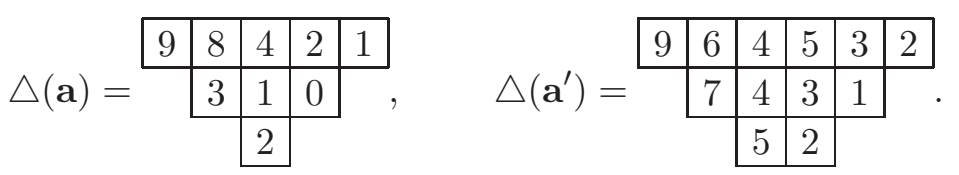


Let $\tilde{\mathrm{c}}\left(t_{i, n-1}\right)=\sum_{k=i}^{n-1} t_{k, n-1}, \quad \tilde{\mathrm{c}}\left(t_{i, n}\right)=\sum_{k=i}^{n-1} t_{k, n}$, and

$$
\mathrm{c}\left(t_{i, j}\right)= \begin{cases}\sum_{k=i}^{n} t_{k, j} & \text { if } j \leq n\left(\mathrm{~A}_{n}\right), j=n\left(\mathrm{~B}_{n}\right), \\ \sum_{k=i}^{n}\left(t_{k, j}+t_{k, 2 n-j}\right) & \text { if } j<n\left(\mathrm{~B}_{n}, \mathrm{C}_{n}\right), \\ \sum_{k=i}^{n-1}\left(t_{k, j}+t_{k, 2 n-1-j}\right) & \text { if } j<n-1\left(\mathrm{D}_{n}\right), \\ \sum_{k=i}^{n} 2 t_{k, j} & \text { if } j=n\left(\mathrm{C}_{n}\right), \\ \sum_{k=i}^{n-1}\left(t_{k, n-1}+t_{k, n}\right) & \text { if } j=n-1, n\left(\mathrm{D}_{n}\right), \\ t_{i, j}+\sum_{k=i+1}^{n}\left(t_{k, 2 n-j}+t_{k, j}\right) & \text { if } j>n\left(\mathrm{~B}_{n}, \mathrm{C}_{n}\right), \\ t_{i, j}+\sum_{k=i+1}^{n-1}\left(t_{k, 2 n-1-j}+t_{k, j}\right) & \text { if } j>n\left(\mathrm{D}_{n}\right) .\end{cases}
$$

Proposition $3.1([24])$. Let $\left(\mathfrak{A}, \mathrm{P}, \Pi, \mathrm{P}^{\vee}, \Pi^{\vee}\right)$ be a Cartan datum of finite classical type, and let $\lambda=\lambda_{1} \Lambda_{1}+\cdots+\lambda_{n} \Lambda_{n} \in \mathrm{P}^{+}$. For $\mathbf{a} \in\left(\mathbb{Z}_{\geq 0}\right)^{\ell}$, let $\triangle(\mathbf{a})=\left\{t_{i j}\right\}_{1 \leq i \leq n^{\prime}, p_{i} \leq j \leq p_{i}^{\prime}}$ be as above and assume $\mathrm{c}\left(t_{i, j}\right)$ is as in (3.1). Then

$$
\begin{aligned}
& \left(\mathrm{A}_{n}\right): \mathcal{S}=\left\{\mathbf{a} \in\left(\mathbb{Z}_{\geq 0}\right)^{\ell} \mid t_{i, n+1-i} \geq t_{i, n+2-i} \geq \cdots \geq t_{i, n} \text { for } 1 \leq i \leq n\right\}, \\
& \mathcal{S}^{\lambda}=\left\{\mathbf{a} \in \mathcal{S} \mid t_{i, j} \leq \lambda_{j}+\mathrm{c}\left(t_{i+1, j-1}\right)-2 \mathrm{c}\left(t_{i+1, j}\right)+\mathrm{c}\left(t_{i, j+1}\right)\right. \\
& \text { for } 1 \leq i \leq n, n+1-i \leq j \leq n\} \\
& \left(\mathrm{B}_{n}\right): \mathcal{S}=\left\{\mathbf{a} \in\left(\mathbb{Z}_{\geq 0}\right)^{\ell} \mid 2 t_{i, n+1-i} \geq \ldots \geq 2 t_{i, n-1} \geq t_{i, n} \geq 2 t_{i, n+1} \geq \ldots \geq 2 t_{i, n-1+i}\right. \\
& \text { for } 1 \leq i \leq n\} \text {, } \\
& \mathcal{S}^{\lambda}=\left\{\mathbf{a} \in \mathcal{S} \mid t_{i, j} \leq \lambda_{j}+\mathrm{c}\left(t_{i, j+1}\right)-2 \mathrm{c}\left(t_{i, 2 n-j}\right)+\mathrm{c}\left(t_{i, 2 n+1-j}\right),\right. \\
& t_{i, 2 n-j} \leq \lambda_{j}+\mathrm{c}\left(t_{i+1, j+1}\right)-2 \mathrm{c}\left(t_{i+1, j}\right)+\mathrm{c}\left(t_{i, 2 n+1-j}\right) \text {, } \\
& \left.t_{i, n} \leq \lambda_{n}+2 \mathrm{c}\left(t_{i, n+1}\right)-2 \mathrm{c}\left(t_{i+1, n}\right) \quad \text { for } 1 \leq i \leq n, n+1-i \leq j<n\right\} \\
& \left(\mathrm{C}_{n}\right): \mathcal{S}=\left\{\mathbf{a} \in\left(\mathbb{Z}_{\geq 0}\right)^{\ell} \mid t_{i, n+1-i} \geq \ldots \geq t_{i, n} \geq \ldots \geq t_{i, n-1+i} \text { for } 1 \leq i \leq n\right\} \\
& \mathcal{S}^{\lambda}=\left\{\mathbf{a} \in \mathcal{S} \mid t_{i, j} \leq \lambda_{j}+\mathrm{c}\left(t_{i, j+1}\right)-2 \mathrm{c}\left(t_{i, 2 n-j}\right)+\mathrm{c}\left(t_{i, 2 n+1-j}\right),\right. \\
& t_{i, 2 n-j} \leq \lambda_{j}+\mathrm{c}\left(t_{i+1, j+1}\right)-2 \mathrm{c}\left(t_{i+1, j}\right)+\mathrm{c}\left(t_{i, 2 n+1-j}\right), \\
& \left.t_{i, n} \leq \lambda_{n}+\mathrm{c}\left(t_{i, n+1}\right)-\mathrm{c}\left(t_{i+1, n}\right) \text { for } 1 \leq i \leq n, n+1-i \leq j<n\right\} \\
& \left(\mathrm{D}_{n}\right): \mathcal{S}=\left\{\mathbf{a} \in\left(\mathbb{Z}_{\geq 0}\right)^{\ell} \mid t_{i, n-i} \geq \ldots \geq t_{i, n-2} \geq t_{i, n-1}, t_{i, n} \geq t_{i, n+1} \geq \ldots \geq t_{i, n-1+i}\right. \\
& \text { for } 1 \leq i \leq n-1\} \text {, } \\
& \mathcal{S}^{\lambda}=\left\{\mathbf{a} \in \mathcal{S} \mid t_{i, j} \leq \lambda_{j}+\mathrm{c}\left(t_{i, j+1}\right)-2 \mathrm{c}\left(t_{i, 2 n-1-j}\right)+\mathrm{c}\left(t_{i, 2 n-j}\right),\right. \\
& t_{i, 2 n-1-j} \leq \lambda_{j}+\mathrm{c}\left(t_{i+1, j+1}\right)-2 \mathrm{c}\left(t_{i+1, j}\right)+\mathrm{c}\left(t_{i, 2 n-j}\right) \text {, } \\
& t_{i, n-1} \leq \lambda_{n-1}+\mathrm{c}\left(t_{i, n+1}\right)-2 \tilde{\mathrm{c}}\left(t_{i+1, n-1}\right) \text {, } \\
& \left.t_{i, n} \leq \lambda_{n}+\mathrm{c}\left(t_{i, n+1}\right)-2 \tilde{\mathrm{c}}\left(t_{i+1, n}\right) \text { for } 1 \leq i \leq n-1, n-i \leq j<n-1\right\} \text {. }
\end{aligned}
$$

Let $\mathbf{B}$ be the crystal given by 
$\left(\mathrm{A}_{n}\right)$

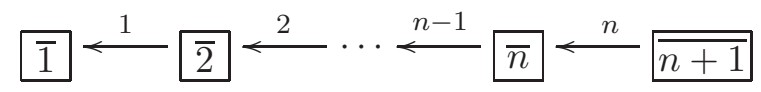

$\left(\mathrm{B}_{n}\right)$

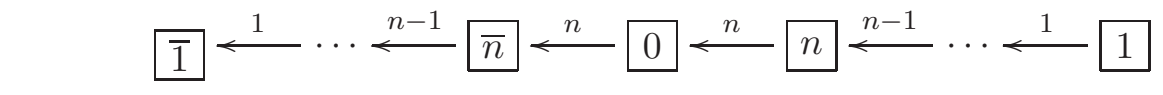

$\left(\mathrm{C}_{n}\right)$

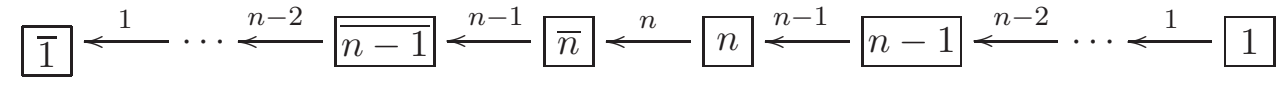

$\left(\mathrm{D}_{n}\right)$

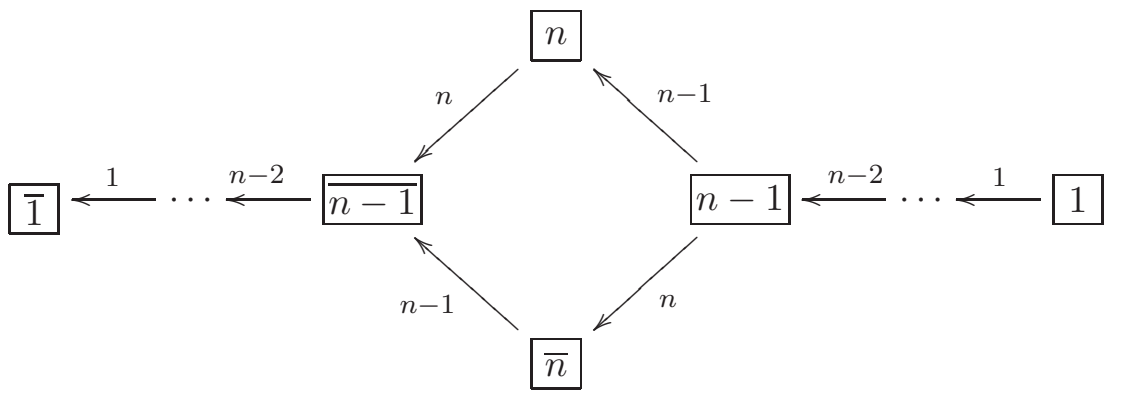

with the entries ordered by
$\left(\mathrm{A}_{n}\right)$
$\overline{1} \succ \overline{2} \succ \cdots \succ \overline{n+1}$,
$\left(\mathrm{B}_{n}\right)$
$\overline{1} \succ \overline{2} \succ \cdots \succ \bar{n} \succ 0 \succ n \succ \cdots \succ 2 \succ 1$,
$\left(\mathrm{C}_{n}\right)$
$\overline{1} \succ \overline{2} \succ \cdots \succ \overline{n-1} \succ \bar{n} \succ n \succ n-1 \succ \cdots \succ 1$,
$\left(\mathrm{D}_{n}\right)$
$\overline{1} \succ \overline{2} \succ \cdots \succ \overline{n-1} \succ \bar{n}, n \succ n-1 \succ \cdots \succ 1$.

Let

$$
\widehat{\imath}= \begin{cases}\bar{\imath} & \text { if } 1 \leq i \leq n+1 \quad\left(\mathrm{~A}_{n}\right), 1 \leq i \leq n \quad\left(\mathrm{~B}_{n}, \mathrm{C}_{n}, \mathrm{D}_{n}\right), \\ 0 & \text { if } i=n+1 \quad\left(\mathrm{~B}_{n}\right), \\ 2 n+1-i & \text { if } n+1 \leq i \leq 2 n\left(\mathrm{C}_{n}, \mathrm{D}_{n}\right), \\ 2 n+2-i & \text { if } n+2 \leq i \leq 2 n+1 \quad\left(\mathrm{~B}_{n}\right) .\end{cases}
$$

For $a, b \in \mathbf{B}$ with $a \succ b$, let $\mathbf{i}(a, b)$ be a sequence of elements in $I$ such that $a=\tilde{f}_{\mathbf{i}(a, b)} b$, and define the irreducible graded $R$-module

$$
\Delta_{(a, b)}=\tilde{f}_{\mathbf{i}(a, b)} \mathbf{1} .
$$

Note that $\Delta_{(a, b)}$ in general is not a cuspidal representation given in [7, 21].

Remark. The set of cuspidal representations is in one-to-one correspondence with the set of positive roots, via correspondence given by the weights of the cuspidal representations. But there are some $\Delta(a, b)$ 's which do not correspond to positive roots. For example, in type $\mathrm{B}_{n}$, the module $\Delta_{(\overline{1}, 1)} \in R\left(\sum_{i=1}^{n} 2 \alpha_{i}\right)$-fmod is not a cuspidal representation, since $\sum_{i=1}^{n} 2 \alpha_{i}$ is not a positive root (see Example 3.5).

The action of the KLR algebra can be described explicitly as follows. 
If one of the following holds: $a \succ b \quad\left(\mathrm{~A}_{n}, \mathrm{C}_{n}\right)$, either $b \succeq 0$ or $0 \succeq a \quad\left(\mathrm{~B}_{n}\right)$, either $b \succ n-1$ or $\overline{n-1} \succ a\left(\mathrm{D}_{n}\right)$, then the module $\Delta_{(a, b)}$ is the 1-dimensional $R$-module $\mathbb{C} v$ specified by

$$
x_{i} v=0, \quad \tau_{j} v=0, \quad e(\mathbf{i}) v= \begin{cases}v & \text { if } \mathbf{i}=\mathbf{i}(a, b), \\ 0 & \text { otherwise. }\end{cases}
$$

If $a \succ 0 \succ b$ for type $\mathrm{B}_{n}$, then $\Delta_{(a, b)}$ is the 2-dimensional $R$-module $\mathbb{C} u \oplus \mathbb{C} v$ with $R$-action

$$
\begin{aligned}
& x_{i} u=0, \quad \tau_{j} u=\left\{\begin{array}{ll}
v & \text { if } j=d, \\
0 & \text { otherwise, }
\end{array} \quad e(\mathbf{i}) u= \begin{cases}u & \text { if } \mathbf{i}=\mathbf{i}(a, b), \\
0 & \text { otherwise, }\end{cases} \right. \\
& x_{i} v=\left\{\begin{array}{ll}
-u & \text { if } i=d, \\
u & \text { if } i=d+1, \\
0 & \text { otherwise }
\end{array} \quad \tau_{j} v=0,\right.
\end{aligned}
$$

where $d$ is an integer such that $\sigma_{d}(\mathbf{i}(a, b))=\mathbf{i}(a, b)$.

If $a \succeq \overline{n-1}$ and $n-1 \succeq b$ for type $\mathrm{D}_{n}$, then the module $\Delta_{(a, b)}$ is the 2-dimensional $R$-module $\mathbb{C} u \oplus \mathbb{C} v$ given by

$$
\begin{array}{ll}
x_{i} u=0, \quad \tau_{j} u=\left\{\begin{array}{ll}
\mathcal{Q}_{n, n-1}\left(x_{n}, x_{n-1}\right) v & \text { if } j=d, \\
0 & \text { otherwise, }
\end{array} \quad e(\mathbf{i}) u= \begin{cases}u & \text { if } \mathbf{i}=\mathbf{i}(a, n) * \mathbf{i}(n, b), \\
0 & \text { otherwise },\end{cases} \right. \\
x_{i} v=0, \quad \tau_{j} v= \begin{cases}u & \text { if } j=d, \\
0 & \text { otherwise, }\end{cases} & e(\mathbf{i}) v= \begin{cases}v & \text { if } \mathbf{i}=\mathbf{i}(a, \bar{n}) * \mathbf{i}(\bar{n}, b), \\
0 & \text { otherwise },\end{cases}
\end{array}
$$

where $d$ is an integer such that $\sigma_{d}(\mathbf{i}(a, n) * \mathbf{i}(n, b))=\mathbf{i}(a, \bar{n}) * \mathbf{i}(\bar{n}, b)$. Note that $\mathcal{Q}_{n, n-1}\left(x_{n}, x_{n-1}\right)=$ $\zeta_{n, n-1} \in \mathbb{C} \backslash\{0\}$.

It follows from the description above that, for $a, b \in \mathbf{B}$ with $a \succ b$,

$$
\operatorname{ch} \Delta_{(a, b)}= \begin{cases}\mathbf{i}(a, n) * \mathbf{i}(n, b)+\mathbf{i}(a, \bar{n}) * \mathbf{i}(\bar{n}, b) & \text { if } a \succeq \overline{n-1}, n-1 \succeq b \quad\left(\mathrm{D}_{n}\right), \\ 2 \mathbf{i}(a, b) & \text { if } a \succ 0 \succ b \quad\left(\mathrm{~B}_{n}\right), \\ \mathbf{i}(a, b) & \text { otherwise. }\end{cases}
$$

Remark. We see from the expression for the character in (3.4) that $\Delta_{(a, b)}$ can be identified with the sequence $\mathbf{i}(a, b)$, or equivalently, with the segment of $\mathbf{B}$ between a and $b$, which is another reason why we defined the Kashiwara operators in the opposite manner to [15, 19].

Given $\mathbf{a} \in \mathcal{S}\left(\right.$ resp. $\left.\mathbf{a} \in \mathcal{S}^{\lambda}\right)$ and $i=1, \ldots, n$, let

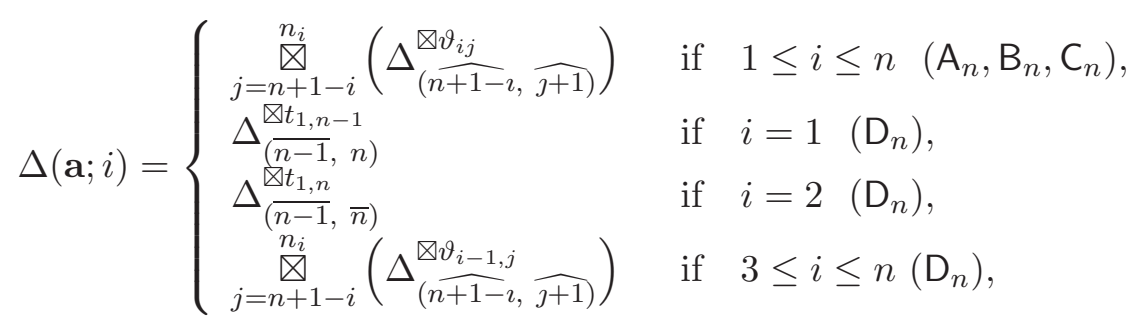


where $n_{i}=n$ for all $i\left(\mathrm{~A}_{n}\right), n_{i}=n+i \quad\left(\mathrm{~B}_{n}\right), n_{i}=n-1+i \quad\left(\mathrm{C}_{n}, \mathrm{D}_{n}\right), \triangle(\mathbf{a})=\left\{t_{i, j}\right\}$, and

$$
\vartheta_{i j}= \begin{cases}t_{i, j}-t_{i, j+1} & \text { if } j \leq n_{i} \quad\left(\mathrm{~A}_{n}, \mathrm{C}_{n}\right), j \leq n-2\left(\mathrm{~B}_{n}\right), j \leq n-3\left(\mathrm{D}_{n}\right), \\ t_{i, n-1}-\left\lceil\frac{t_{i, n}}{2}\right\rceil & \text { if } j=n-1 \quad\left(\mathrm{~B}_{n}\right), \\ \left\lceil\frac{t_{i, n}}{2}\right\rceil-\left\lfloor\frac{t_{i, n}}{2}\right\rfloor & \text { if } j=n\left(\mathrm{~B}_{n}\right), \\ \left\lfloor\frac{t_{i, n}}{2}\right\rfloor-t_{i, n+1} & \text { if } j=n+1\left(\mathrm{~B}_{n}\right), \\ t_{i, n-2}-\max \left\{t_{i, n-1}, t_{i, n}\right\} & \text { if } j=n-2\left(\mathrm{D}_{n}\right), \\ \max \left\{0, t_{i, n}-t_{i, n-1}\right\} & \text { if } j=n-1\left(\mathrm{D}_{n}\right), \\ \max \left\{0, t_{i, n-1}-t_{i, n}\right\} & \text { if } j=n\left(\mathrm{D}_{n}\right), \\ \min \left\{t_{i, n-1}, t_{i, n}\right\}-t_{i, n+1} & \text { if } j=n+1\left(\mathrm{D}_{n}\right), \\ t_{i, j-1}-t_{i, j} & \text { if } j \geq n+2\left(\mathrm{~B}_{n}, \mathrm{D}_{n}\right) .\end{cases}
$$

For $v \in B(\infty)$ (resp. $v \in B(\lambda)$ ), let $\mathbf{a}(v)$ be the adapted string of $v$ with respect to the expression $w_{0}=r_{\mathbf{s}_{1}} \cdots r_{\mathbf{s}_{n}}$ given in Table 1. Now we are ready to state the main theorem in this section.

Theorem 3.2. Let $\left(\mathfrak{A}, \mathrm{P}, \Pi, \mathrm{P}^{\vee}, \Pi^{\vee}\right)$ be a Cartan datum of finite classical type. For $v \in$ $B(\infty)$ (resp. $v \in B(\lambda)$ ), let $\mathbf{a}(v)$ be the adapted string of $v$ with respect to the expression $w_{0}=r_{\mathbf{s}_{1}} \cdots r_{\mathbf{s}_{n}}$ given in Table 1 and let $\Delta(\mathbf{a}(v))=\Delta(\mathbf{a}(v) ; 1) \otimes \cdots \otimes \Delta(\mathbf{a}(v) ; n)$. Then

(1) hd Ind $\Delta(\mathbf{a}(v))$ is irreducible.

(2) The map $\Psi: B(\infty) \longrightarrow \mathbb{B}(\infty)$ defined by

$$
\Psi(v)=\operatorname{hd} \operatorname{lnd} \Delta(\mathbf{a}(v)) \quad \text { for } v \in B(\infty)
$$

is a crystal isomorphism.

(3) The map $\Psi^{\lambda}: B(\lambda) \longrightarrow \mathbb{B}(\lambda)$ defined by

$$
\Psi^{\lambda}(v)=\operatorname{hd} \operatorname{lnd} \Delta(\mathbf{a}(v)) \quad \text { for } v \in B(\lambda)
$$

is a crystal isomorphism.

Theorem 3.2 and Proposition 3.1 combine to give the following explicit description of the irreducible graded modules over $R$ and $R^{\lambda}$.

Corollary 3.3. Let $\left(\mathfrak{A}, \mathrm{P}, \Pi, \mathrm{P}^{\vee}, \Pi^{\vee}\right)$ be a Cartan datum of finite classical type. Then

(1) the set

$$
\mathcal{A}=\{\operatorname{hdlnd} \Delta(\mathbf{a}) \mid \mathbf{a} \in \mathcal{S}\}
$$

is the complete list of all irreducible graded $R$-modules up to isomorphism and grading shift. 
(2) For $\lambda \in \mathrm{P}^{+}$, the set

$$
\mathcal{A}^{\lambda}=\left\{\operatorname{hd} \operatorname{lnd} \Delta(\mathbf{a}) \mid \mathbf{a} \in \mathcal{S}^{\lambda}\right\}
$$

is the complete list of all irreducible graded $R^{\lambda}$-modules up to isomorphism and grading shift.

In the following proposition, we give an upper bound for the number of $\Delta_{(a, b)}$ 's that can occur in $\Delta(\mathbf{a}(v))$ for $v \in B(\lambda)$.

Proposition 3.4. For $v \in B(\lambda)$, let $\eta(v)$ be the number of $\Delta_{(a, b)}$ 's in the outer tensor product $\Delta(\mathbf{a}(v))=\Delta(\mathbf{a}(v) ; 1) \otimes \cdots \otimes \Delta(\mathbf{a}(v) ; n)$. Then $\eta(v) \leq n \lambda(h)$, where

$$
h= \begin{cases}h_{1}+\cdots+h_{n} & \left(\mathrm{~A}_{n}\right), \\ 2 h_{1}+\cdots+2 h_{n-1}+h_{n} & \left(\mathrm{~B}_{n}\right), \\ 2\left(h_{1}+\cdots+h_{n}\right) & \left(\mathrm{C}_{n}\right), \\ 2 h_{1}+\cdots+2 h_{n-2}+h_{n-1}+h_{n} & \left(\mathrm{D}_{n}\right) .\end{cases}
$$

Proof. Let $\mathbf{a}=\mathbf{a}(v)$ and write $\mathbf{a}=\mathbf{a}_{1} * \cdots * \mathbf{a}_{n}$, where $\mathbf{a}_{k}=\left(\mathrm{a}_{k, 1}, \mathrm{a}_{k, 2}, \ldots, \mathrm{a}_{k, l_{k}}\right)$ is the subsequence of a defined in (2.3). Since $\mathrm{a}_{k, 1}=t_{k, n+1-k}$ if $\mathfrak{A}$ is of type $\mathrm{A}_{n}, \mathrm{~B}_{n}, \mathrm{C}_{n}$ and $\mathrm{a}_{1,1}=t_{1, n-1}, \mathrm{a}_{2,1}=t_{1, n}, \mathrm{a}_{k, 1}=t_{k-1, n+1-k}$ when $n \geq 3$ for type $\mathrm{D}_{n}$, it follows from (3.5) and (3.6) that

$$
\eta(v) \leq \mathrm{a}_{1,1}+\cdots+\mathrm{a}_{n-1,1}+\mathrm{a}_{n, 1} .
$$

Thus, it suffices to show that

$$
\mathrm{a}_{1,1}+\cdots+\mathrm{a}_{n-1,1}+\mathrm{a}_{n, 1} \leq n \lambda(h)
$$

Let $\lambda=\lambda_{1} \Lambda_{1}+\cdots+\lambda_{n} \Lambda_{n}$. If $n>1$ for type $\mathrm{A}_{n}, \mathrm{~B}_{n}, \mathrm{C}_{n}$ and $n>2$ for type $\mathrm{D}_{n}$, then using the description in Proposition 3.1, we will obtain

$$
\mathrm{a}_{n, 1} \leq \lambda(h)
$$

from the following inequalities: 


$$
\begin{aligned}
\left(\mathrm{A}_{n}\right): \mathrm{a}_{n, 1}=t_{n, 1} & \leq \lambda_{1}+t_{n, 2} \leq \lambda_{1}+\lambda_{2}+t_{n, 3} \leq \cdots \leq \lambda_{1}+\cdots+\lambda_{n}=\lambda(h), \\
\left(\mathrm{B}_{n}\right): \mathrm{a}_{n, 1}=t_{n, 1} & \leq \lambda_{1}+t_{n, 2}+t_{n, 2 n-2}-2 t_{n, 2 n-1} \leq \lambda_{1}+\lambda_{2}+t_{n, 3}+t_{n, 2 n-3}-t_{n, 2 n-2}-t_{n, 2 n-1} \\
& \leq \cdots \leq \lambda_{1}+\cdots+\lambda_{n-1}+t_{n, n}-t_{n, n+1}-t_{n, 2 n-1} \\
& \leq \lambda_{1}+\cdots+\lambda_{n}+t_{n, n+1}-t_{n, 2 n-1} \leq \lambda_{1}+\cdots+\lambda_{n}+\lambda_{n-1}+t_{n, n+2}-t_{n, 2 n-1} \\
& \leq \cdots \leq 2 \lambda_{1}+2 \lambda_{2}+\cdots+2 \lambda_{n-1}+\lambda_{n}=\lambda(h), \\
\left(\mathrm{C}_{n}\right): \mathrm{a}_{n, 1}=t_{n, 1} & \leq \lambda_{1}+t_{n, 2}+t_{n, 2 n-2}-2 t_{n, 2 n-1} \leq \lambda_{1}+\lambda_{2}+t_{n, 3}+t_{n, 2 n-3}-t_{n, 2 n-2}-t_{n, 2 n-1} \\
& \leq \cdots \leq \lambda_{1}+\cdots+\lambda_{n-1}+2 t_{n, n}-t_{n, n+1}-t_{n, 2 n-1} \\
& \leq \lambda_{1}+\cdots+\lambda_{n-1}+2 \lambda_{n}+t_{n, n+1}-t_{n, 2 n-1} \\
& \leq \cdots \leq 2 \lambda_{1}+2 \lambda_{2}+\cdots+2 \lambda_{n-1}+2 \lambda_{n}=\lambda(h), \\
\left(\mathrm{D}_{n}\right): \mathrm{a}_{n, 1}=t_{n-1,1} & \leq \lambda_{1}+t_{n-1,2}+t_{n-1,2 n-3}-2 t_{n-1,2 n-2} \\
& \leq \lambda_{1}+\lambda_{2}+t_{n-1,3}+t_{n-1,2 n-4}-t_{n-1,2 n-3}-t_{n-1,2 n-2} \\
& \leq \cdots \leq \lambda_{1}+\cdots+\lambda_{n-2}+t_{n-1, n-1}+t_{n-1, n}-t_{n-1, n+1}-t_{n-1,2 n-2} \\
& \leq \lambda_{1}+\cdots+\lambda_{n}+t_{n-1, n+1}-t_{n-1,2 n-2} \\
& \leq \lambda_{1}+\cdots+\lambda_{n}+\lambda_{n-2}+t_{n-1, n+2}-t_{n-1,2 n-2} \\
& \leq \cdots \leq 2 \lambda_{1}+2 \lambda_{2}+\cdots+2 \lambda_{n-2}+\lambda_{n-1}+\lambda_{n}=\lambda(h) .
\end{aligned}
$$

We proceed by induction on $n$. If $n=2$ for type $\mathrm{A}_{n}, \mathrm{~B}_{n}, \mathrm{C}_{n}$ or $n=3$ for type $\mathrm{D}_{n}$, then the assertion can be proved in the same manner as above. We assume that $n>2$ for type $\mathrm{A}_{n}, \mathrm{~B}_{n}, \mathrm{C}_{n}$ and $n>3$ for type $\mathrm{D}_{n}$. Let $U_{q}\left(\mathfrak{g}_{n-1}\right)$ be the subalgebra of $U_{q}(\mathfrak{g})$ generated by $e_{i}, f_{i}$ $(i \in I \backslash\{1\})$ and $q^{h}\left(h \in \mathrm{P}^{\vee}\right)$, and let $\mathfrak{B}$ be the crystal obtained from $B(\lambda)$ by forgetting the 1-arrows. Note that $U_{q}\left(\mathfrak{g}_{n-1}\right)$ is of type $\mathrm{X}_{n-1}$ when $U_{q}(\mathfrak{g})$ is of type $\mathbf{X}_{n}(\mathrm{X}=\mathrm{A}, \mathrm{B}, \mathrm{C}, \mathrm{D})$. Let $u=\tilde{f}_{\mathbf{s}_{n}}^{\mathrm{a}_{n}} b_{\lambda}$ where $\mathbf{s}_{n}$ is the sequence given in Table 1, and let $\mathcal{C}$ be the connected component of $\mathfrak{B}$ containing $v$. Then $u$ is the highest weight vector of the $U_{q}\left(\mathfrak{g}_{n-1}\right)$-crystal $\mathcal{C}$ with weight

$$
\operatorname{wt}(u)=\left\{\begin{array}{lr}
\lambda-t_{n, 1} \alpha_{1}-\cdots-t_{n, n} \alpha_{n} & \left(\mathrm{~A}_{n}\right), \\
\lambda-\left(t_{n, 1}+t_{n, 2 n-1}\right) \alpha_{1}-\cdots-\left(t_{n, n-1}+t_{n, n+1}\right) \alpha_{n-1}-\left(t_{n, n}\right) \alpha_{n} & \left(\mathrm{~B}_{n}, \mathrm{C}_{n}\right), \\
\lambda-\left(t_{n-1,1}+t_{n-1,2 n-2}\right) \alpha_{1}-\cdots-\left(t_{n-1, n-2}+t_{n-1, n+1}\right) \alpha_{n-2} & \\
-t_{n-1, n} \alpha_{n-1}-t_{n-1, n-1} \alpha_{n} & \left(\mathrm{D}_{n}\right) .
\end{array}\right.
$$

Now suppose that $h^{\prime}=h-h_{1}\left(\mathrm{~A}_{n}\right)$, and $h^{\prime}=h-2 h_{1}\left(\mathrm{~B}_{n}, \mathrm{C}_{n}, \mathrm{D}_{n}\right)$. Then we see that

$$
\begin{array}{lll}
\alpha_{1}\left(h^{\prime}\right)=-1, \alpha_{2}\left(h^{\prime}\right)=1, & \alpha_{i}\left(h^{\prime}\right)=0(i=3, \ldots, n) & \left(\mathrm{A}_{n}\right), \\
\alpha_{1}\left(h^{\prime}\right)=-2, \alpha_{2}\left(h^{\prime}\right)=2, & \alpha_{i}\left(h^{\prime}\right)=0(i=3, \ldots, n) & \left(\mathrm{B}_{n}, \mathrm{C}_{n}, \mathrm{D}_{n}\right) .
\end{array}
$$


Proposition 3.1 implies

$$
\operatorname{wt}(u)\left(h^{\prime}\right) \leq \lambda(h)
$$

by the following calculations:

$$
\begin{aligned}
\left(\mathrm{A}_{n}\right): \operatorname{wt}(u)\left(h^{\prime}\right) & =\lambda\left(h^{\prime}\right)+t_{n, 1}-t_{n, 2} \leq \lambda(h), \\
\left(\mathrm{B}_{n}, \mathrm{C}_{n}\right): \operatorname{wt}(u)\left(h^{\prime}\right) & =\lambda\left(h^{\prime}\right)+2\left(t_{n, 1}+t_{n, 2 n-1}\right)-2\left(t_{n, 2}+t_{n, 2 n-2}\right) \\
& =\lambda\left(h^{\prime}\right)+2\left(t_{n, 1}-t_{n, 2}-t_{n, 2 n-2}+2 t_{n, 2 n-1}\right)-2 t_{n, 2 n-1} \leq \lambda(h), \\
\left(\mathrm{D}_{n}\right): \operatorname{wt}(u)\left(h^{\prime}\right) & =\lambda\left(h^{\prime}\right)+2\left(t_{n, 1}+t_{n, 2 n-2}\right)-2\left(t_{n, 2}+t_{n, 2 n-3}\right) \\
& =\lambda\left(h^{\prime}\right)+2\left(t_{n, 1}-t_{n, 2}-t_{n, 2 n-3}+2 t_{n, 2 n-2}\right)-2 t_{n, 2 n-2} \leq \lambda(h) .
\end{aligned}
$$

Since the adapted string of $u$ is $\mathbf{a}_{1} * \cdots * \mathbf{a}_{n-1}$, we obtain by the induction hypothesis that

$$
\mathrm{a}_{1,1}+\cdots+\mathrm{a}_{n-1,1} \leq(n-1) \mathrm{wt}(u)\left(h^{\prime}\right),
$$

which yields, by (3.7) and (3.8),

$$
\mathrm{a}_{1,1}+\cdots+\mathrm{a}_{n-1,1}+\mathrm{a}_{n, 1} \leq(n-1) \lambda(h)+\lambda(h)=n \lambda(h) .
$$

Example 3.5. Assume $\left(\mathfrak{A}, \mathrm{P}, \Pi, \mathrm{P}^{\vee}, \Pi^{\vee}\right)$ is type $\mathrm{B}_{3}$ and $\lambda=\Lambda_{1}+\Lambda_{2}+3 \Lambda_{3} \in \mathrm{P}^{+}$. In this case, $w_{0}=r_{3}\left(r_{2} r_{3} r_{2}\right)\left(r_{1} r_{2} r_{3} r_{2} r_{1}\right)$ and

$$
\mathbf{B}=\overline{1} \stackrel{1}{\longleftarrow} \overline{2} \stackrel{2}{\longleftarrow}\left[\overline{3} \stackrel{3}{\longleftarrow} 0 \stackrel{3}{\longleftarrow} \stackrel{2}{\longleftarrow}^{\longleftarrow} \stackrel{1}{\longleftarrow} 1 .\right.
$$

Kashiwara and Nakashima [18] constructed combinatorial realizations of highest weight crystals for finite classical types using certain semistandard tableaux, which are now referred to as Kashiwara-Nakashima tableaux. We take this combinatorial model as a realization of the crystal $B(\lambda)$. Let $T$ denote the following element in $B(\lambda)$

$$
T=\begin{array}{|l|l|l|l|}
\hline 1 & 3 & \overline{3} & \overline{1} \\
\hline \overline{3} & 0 & \overline{1} & \\
\hline 2 & \overline{3} &
\end{array}
$$

Since $w_{0}=r_{3}\left(r_{2} r_{3} r_{2}\right)\left(r_{1} r_{2} r_{3} r_{2} r_{1}\right)$, using the crystal structure given in [18, we have

$$
\mathbf{a}(T)=(3,3,3,0,4,3,5,2,1) \in \mathcal{S}^{\lambda}, \quad \triangle(\mathbf{a}(T))=\begin{array}{l|l|l|l|l|}
\hline 4 & 3 & 5 & 2 & 1 \\
\hline & 3 & 3 & 0 & \\
\hline & 3 & &
\end{array}
$$




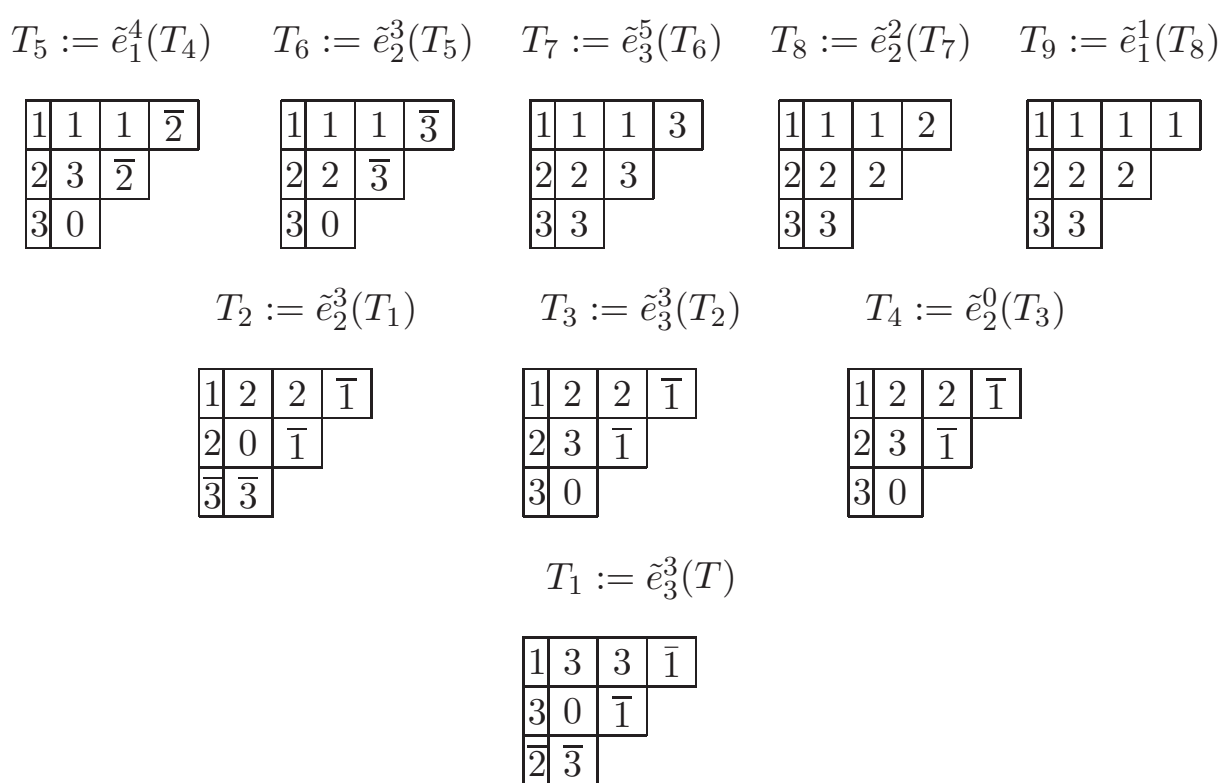

Note that $\varepsilon_{i}\left(T_{9}\right)=0(i=1,2,3), \varepsilon_{i}\left(T_{4}\right)=0(i=2,3)$ and $\varepsilon_{i}\left(T_{1}\right)=0(i=3)$. It follows from (3.5) and (3.6) that

$$
\begin{aligned}
& \vartheta_{1,3}=1, \vartheta_{1,4}=1, \\
& \vartheta_{2,2}=1, \vartheta_{2,3}=1, \vartheta_{2,4}=1, \vartheta_{2,5}=0, \\
& \vartheta_{3,1}=1, \vartheta_{3,2}=0, \vartheta_{3,3}=1, \vartheta_{3,4}=0, \vartheta_{3,5}=1, \vartheta_{3,6}=1,
\end{aligned}
$$

and using the definition of $\widehat{\imath}$ in (3.2), we have

$$
\begin{aligned}
& \Delta(\mathbf{a}(T) ; 1)=\Delta_{(\widehat{3}, \widehat{4})}^{\otimes \vartheta_{1,3}} \otimes \Delta_{(\widehat{3}, 5)}^{\otimes \vartheta_{1,4}} \\
& =\Delta_{(\overline{3}, 0)} \otimes \Delta_{(\overline{3}, 3)}, \\
& \Delta(\mathbf{a}(T) ; 2)=\Delta_{(\widehat{2}, \widehat{3})}^{\otimes \vartheta_{2,2}} \otimes \Delta_{(\widehat{2}, \widehat{4})}^{\otimes \vartheta_{2,3}} \otimes \Delta_{(\widehat{2}, \widehat{5})}^{\otimes \vartheta_{2,4}} \otimes \Delta_{(\widehat{2}, \widehat{6})}^{\otimes \vartheta_{2,5}} \\
& =\Delta_{(\overline{2}, \overline{3})} \otimes \Delta_{(\overline{2}, 0)} \otimes \Delta_{(\overline{2}, 3)},
\end{aligned}
$$

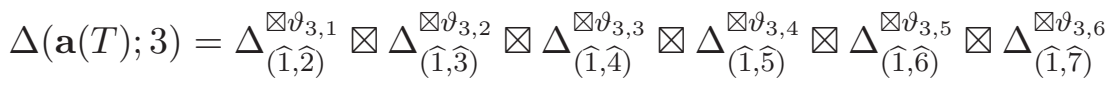

$$
\begin{aligned}
& =\Delta_{(\overline{1}, \overline{2})} \otimes \Delta_{(\overline{1}, 0)} \otimes \Delta_{(\overline{1}, 2)} \otimes \Delta_{(\overline{1}, 1)} .
\end{aligned}
$$

By Theorem 3.2 , the $R^{\lambda}$-module $M$ corresponding to $T$ is given as follows:

$$
\begin{aligned}
M & =\Psi^{\lambda}(T) \\
& =\text { hd Ind }(\Delta(\mathbf{a}(T) ; 1) \otimes \Delta(\mathbf{a}(T) ; 2) \otimes \Delta(\mathbf{a}(T) ; 3)) \\
& =\text { hd Ind }\left(\Delta_{(\overline{3}, 0)} \otimes \Delta_{(\overline{3}, 3)} \otimes \Delta_{(\overline{2}, \overline{3})} \otimes \Delta_{(\overline{2}, 0)} \otimes \Delta_{(\overline{2}, 3)} \otimes \Delta_{(\overline{1}, \overline{2})} \otimes \Delta_{(\overline{1}, 0)} \otimes \Delta_{(\overline{1}, 2)} \otimes \Delta_{(\overline{1}, 1)}\right) .
\end{aligned}
$$

In this example, $\eta(T)=9<n \lambda(h)=3\left(\Lambda_{1}+\Lambda_{2}+3 \Lambda_{3}\right)\left(2 h_{1}+2 h_{2}+h_{3}\right)=21$. 
Recall the definition of $\mathcal{N}_{k}(T)$ in (1.4) (here the element $v$ in $B(\lambda)$ is the tableau $T$ ). Then $\mathcal{N}_{1}(T)=\operatorname{hd} \operatorname{Ind}(\Delta(\mathbf{a}(T) ; 1)), \quad \mathcal{N}_{2}(T)=\operatorname{hd} \operatorname{Ind}(\Delta(\mathbf{a}(T) ; 2)), \quad \mathcal{N}_{3}(T)=\operatorname{hd} \operatorname{lnd}(\Delta(\mathbf{a}(T) ; 3))$

We will prove that such a realization of these modules exists for all finite classical types in the next section (Lemma 4.3). In this example, we give an intuitive picture for the case of $\mathcal{N}_{3}(T)$. For $a, b \in \mathbf{B}$ with $a \succ b$, by the definition of $\Delta_{(a, b)}$ in (3.3) we can identify $\Delta_{(a, b)}$ with the segment of $\mathbf{B}$ between $a$ and $b$. Then we have the following diagram.

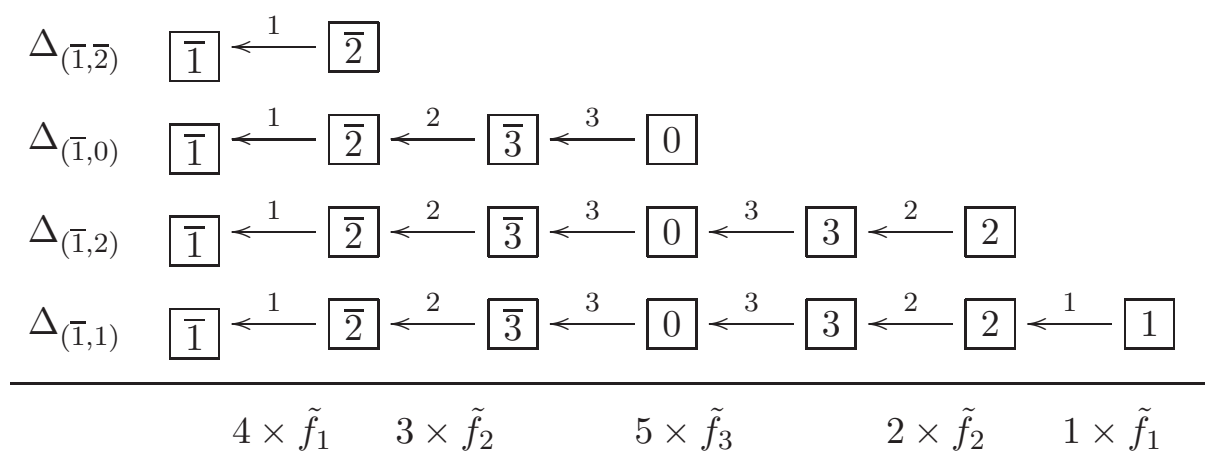

The Kashiwara operators at the bottom of the diagram are obtained by adding up vertically the number of $i$-colored arrows in the segments. By Proposition 2.3, we know

$$
\mathcal{N}_{3}(T)=\tilde{f}_{1}^{4} \tilde{f}_{2}^{3} \tilde{f}_{3}^{5} \tilde{f}_{2}^{2} \tilde{f}_{1}^{1} \mathbf{1}
$$

Since $\mathcal{N}_{3}(T)=$ hd Ind $\left(\Delta_{(\overline{1}, \overline{2})} \otimes \Delta_{(\overline{1}, 0)} \otimes \Delta_{(\overline{1}, 2)} \otimes \Delta_{(\overline{1}, 1)}\right)$, taking the head of Ind $\left(\Delta_{(\overline{1}, \overline{2})} \otimes \Delta_{(\overline{1}, 0)} \otimes\right.$ $\left.\Delta_{(\overline{1}, 2)} \otimes \Delta_{(\overline{1}, 1)}\right)$ can be understood as summing vertically the $i$-colored arrows in the segments corresponding to the modules $\Delta_{(\overline{1}, \overline{2})}, \Delta_{(\overline{1}, 0)}, \Delta_{(\overline{1}, 2)}$ and $\Delta_{(\overline{1}, 1)}$.

Now from the crystal structure in [18], we know that

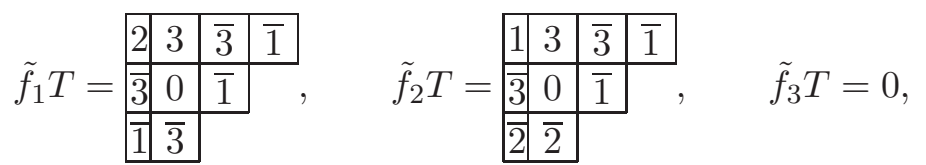

which yield $\mathbf{a}\left(\tilde{f}_{1} T\right)=(3,2,1,0,5,4,7,2,1) \in \mathcal{S}^{\lambda}, \mathbf{a}\left(\tilde{f}_{2} T\right)=(3,4,3,0,4,3,5,2,1) \in \mathcal{S}^{\lambda}$ and

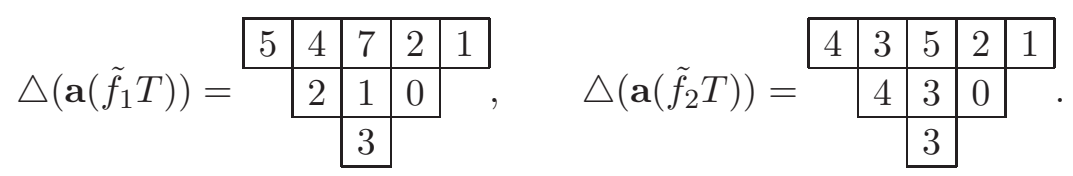


Therefore, by Theorem 3.2 , the irreducible modules $\tilde{f}_{1}(M)=$ hd Ind $(L(1) \otimes M)$ and $\tilde{f}_{2}(M)=$ hd Ind $(L(2) \otimes M)$ can be gotten as follows:

$$
\begin{aligned}
\tilde{f}_{1}(M) & =\tilde{f}_{1}\left(\Psi^{\lambda}(T)\right)=\Psi^{\lambda}\left(\tilde{f}_{1} T\right) \\
& =\text { hd Ind }\left(\Delta_{(\overline{3}, 0)} \otimes \Delta_{(\overline{3}, 3)} \otimes \Delta_{(\overline{2}, \overline{3})} \otimes \Delta_{(\overline{2}, 0)} \otimes \Delta_{(\overline{1}, \overline{2})} \otimes \Delta_{(\overline{1}, 0)} \otimes \Delta_{(\overline{1}, 3)} \otimes \Delta_{(\overline{1}, 2)} \otimes \Delta_{(\overline{1}, 1)}\right), \\
\tilde{f}_{2}(M) & =\tilde{f}_{2}\left(\Psi^{\lambda}(T)\right)=\Psi^{\lambda}\left(\tilde{f}_{2} T\right) \\
& =\operatorname{hd} \operatorname{lnd}\left(\Delta_{(\overline{3}, 0)} \otimes \Delta_{(\overline{3}, 3)} \otimes \Delta_{(\overline{2}, \overline{3})}^{\otimes 2} \otimes \Delta_{(\overline{2}, 0)} \otimes \Delta_{(\overline{2}, 3)} \otimes \Delta_{(\overline{1}, \overline{2})} \otimes \Delta_{(\overline{1}, 0)} \otimes \Delta_{(\overline{1}, 2)} \otimes \Delta_{(\overline{1}, 1)}\right) .
\end{aligned}
$$

Note that by the same argument as above, one can compute the action of Kashiwara operators on $\mathbb{B}(\infty)$ explicitly using the combinatorial realizations of $B(\infty)$ for finite classical type in [5, 9].

\section{Proof of Theorem 3.2}

This section is devoted to a proof of Theorem 3.2. We first give a sufficient condition for Ind $\left(\Delta_{(a, b)} \otimes \Delta_{(c, d)}\right) \simeq$ Ind $\left(\Delta_{(c, d)} \otimes \Delta_{(a, b)}\right)$ to hold (Lemma 4.1). Then we extend the result in [15, Lem. 4.3] for type $A_{n}$ to the other finite classical types in Lemma 4.2 below, and prove Lemma 4.3 which yields $\mathcal{N}_{n}(v)=$ hd Ind $\Delta(\mathbf{a}(v) ; n)$ for $v \in B(\infty)$. From that we can deduce $\mathcal{N}_{k}(v)=\operatorname{hd} \operatorname{lnd} \Delta(\mathbf{a}(v) ; k)$ for all $k$. Using Lemma 1.8, Proposition 1.10, and Lemma 4.3, we argue that the map $\Psi: B(\infty) \longrightarrow \mathbb{B}(\infty)$ is a crystal isomorphism. It then follows from the crystal embedding $\iota_{\lambda}: B(\lambda) \rightarrow B(\infty) \otimes T_{\lambda} \otimes C$ in (1.1) that the map $\Psi^{\lambda}: B(\lambda) \longrightarrow \mathbb{B}(\lambda)$ is a crystal isomorphism too.

Given $\mathbf{i} \in I^{\alpha}$ and $\mathbf{j} \in I^{\beta}$, a sequence $\mathbf{k} \in I^{\alpha+\beta}$ is called a shuffle of $\mathbf{i}$ and $\mathbf{j}$ if $\mathbf{k}$ is a permutation of $\mathbf{i} * \mathbf{j}$ such that $\mathbf{i}$ and $\mathbf{j}$ are subsequences of $\mathbf{k}$. For $X=\sum x_{\mathbf{i}} \mathbf{i}$ and $Y=\sum y_{\mathbf{j}} \mathbf{j}$, we define $X \star Y$ by

$$
X \star Y=\sum_{\mathbf{k}} x_{\mathbf{i}} y_{\mathbf{j}} \mathbf{k}
$$

where $\mathbf{k}$ runs over all the shuffles of $\mathbf{i}$ and $\mathbf{j}$. Suppose $M \in R(\alpha)$-fmod and $N \in R(\beta)$-fmod, and assume $Q$ is a quotient (resp. $L$ is a submodule) of $\operatorname{Ind}(M \otimes N)$. Then

$$
\operatorname{ch}(\operatorname{Ind}(M \otimes N))=\operatorname{ch}(M) \star \operatorname{ch}(N),
$$

any term of $\operatorname{ch} Q$ (resp. $\operatorname{ch} L$ ) is a shuffle of some $\mathbf{i} \in \operatorname{ch}(M)$ and $\mathbf{j} \in \operatorname{ch}(N)$. 
The quantum Serre relations (1.3) imply that

$$
\begin{array}{ll}
\operatorname{dim}(e(\ldots, i, j, \ldots) M)=\operatorname{dim}(e(\ldots, j, i, \ldots) M) & \text { if } a_{i j}=0, \\
2 \operatorname{dim}(e(\ldots, i, j, i, \ldots) M) & \\
\quad=\operatorname{dim}(e(\ldots, i, i, j, \ldots) M)+\operatorname{dim}(e(\ldots, j, i, i, \ldots) M) & \text { if } a_{i j}=-1, \\
\operatorname{dim}(e(\ldots, i, i, i, j, \ldots) M)+3 \operatorname{dim}(e(\ldots, i, j, i, i, \ldots) M) & \\
\quad=\operatorname{dim}(e(\ldots, j, i, i, i, \ldots) M)+3 \operatorname{dim}(e(\ldots, i, i, j, i, \ldots) M) & \text { if } a_{i j}=-2,
\end{array}
$$

for $M \in R$-fmod.

Using the description of $\Delta_{(a, b)}$ in Section 3, we obtain a surjective homomorphism

$$
\text { Ind }\left(\Delta_{(a, b)} \otimes \Delta_{(b, c)}\right) \rightarrow \Delta_{(a, c)}
$$

for $a, b, c \in \mathbf{B}$ with $a \succ b \succ c$.

Lemma 4.1. Let $a, b, c, d \in \mathbf{B}$ with $a \succeq c \succ d \succeq b$. Suppose that one of the following conditions hold:

(1) $\mathfrak{A}$ is of type $\mathrm{A}_{n}$,

(2) $\mathfrak{A}$ is of type $\mathrm{B}_{n}$ and one of the following three holds:

(i) $0 \succeq a$ and $c \neq 0$,

(ii) $b \succeq 0$ and $d \neq 0$,

(iii) $a \succeq \bar{n}, n \succeq b$ and either $c=0$ or $d=0$,

(3) $\mathfrak{A}$ is of type $\mathrm{C}_{n}$ and one of the following three holds:

(i) $b \succeq \bar{n}$,

(ii) $a=c=\bar{n}$,

(iii) $a=\overline{1}$ and $c=\bar{n}$,

(4) $\mathfrak{A}$ is of type $D_{n}$ and one of the following three holds:

(i) $b \succeq \overline{n-1}$,

(ii) $a=c=\overline{n-1}$,

(iii) $a=\overline{1}$ and $c=\overline{n-1}$.

Then

$$
\operatorname{lnd}\left(\Delta_{(a, b)} \otimes \Delta_{(c, d)}\right) \simeq \operatorname{lnd}\left(\Delta_{(c, d)} \otimes \Delta_{(a, b)}\right)
$$

and these modules are irreducible.

Proof. Let

$$
M=\operatorname{lnd}\left(\Delta_{(a, b)} \otimes \Delta_{(c, d)}\right), \quad N=\operatorname{lnd}\left(\Delta_{(c, d)} \otimes \Delta_{(a, b)}\right),
$$

and let $Q$ (resp. $L$ ) be any nonzero quotient (resp. submodule) of $M$. We will assume that $\mathbf{k}$ is a certain sequence of elements in $I$ such that $\mathbf{k} \in \operatorname{ch} M, \mathbf{k} \in \operatorname{ch} Q$, and $\mathbf{k} \in \operatorname{ch} L$. Let 
$\xi_{M}$ (resp. $\left.\xi_{Q}, \xi_{L}\right)$ be the multiplicity of $\mathbf{k}$ in $\operatorname{ch} M($ resp. $\operatorname{ch} Q, \operatorname{ch} L)$. If we can show that $\xi_{M}=\xi_{Q}=\xi_{L}$, we can conclude that $M$ is irreducible and $M \simeq N$, since $\operatorname{ch} M=\operatorname{ch} N$.

For type $\mathrm{A}_{n}$, the assertion follows from [15, Lem. 4.1]. We assume that $\mathfrak{A}$ is of type $\mathrm{B}_{n}$. If either (i) or (ii) holds, the proof is identical to that for type $A_{n}$. So suppose that (iii) holds. Without loss of generality, we may assume that $d=0$. Write $\mathbf{i}=\mathbf{i}(a, b)=(i, \ldots, n, n, \ldots, j)$, $\mathbf{j}=\mathbf{i}(c, d)=(p, p+1, \ldots, n-1, n)$, and let

$$
\mathbf{k}=(i, \ldots, p, p, p+1, p+1, \ldots, n-1, n-1, n, n, n, n-1, n-2, \ldots, j) .
$$

Note that $\mathbf{k}$ is a shuffle of $\mathbf{i}$ and $\mathbf{j}$. Let $l$ be the length of $\mathbf{j}$. It follows from (3.4) and (4.1) that the term $\mathbf{k}$ appears in $\operatorname{ch} M$ with multiplicity $\xi_{M}=2 \times 2^{l-1} 3$.

By Lemma 1.5 and (3.4), $\mathbf{i} * \mathbf{j}$ appears in the character ch $Q$ of any quotient module $Q \subseteq M$. We claim that $\mathbf{k}$ also appears in $\operatorname{ch} Q$. Note that $a_{n, n-1}=-2$ for type $\mathrm{B}_{n}$.

We first assume that $p \neq n-1, n$. By the quantum Serre relations (1.3), if $j>p+1$, then

$$
\begin{aligned}
& \mathbf{i} * \mathbf{j}=(i, \ldots, n, n, \ldots, j, p, p+1, \ldots, n) \in \operatorname{ch} Q, \\
& \stackrel{(4.3)}{\Rightarrow}(i, \ldots, p, p+1, p, \ldots, n, n, \ldots, j, p+1, \ldots, n) \in \operatorname{ch} Q, \\
& \stackrel{(4.2),(4.4)}{\Longrightarrow}(i, \ldots, p, p, p+1, \ldots, n, n, \ldots, j, p+1, \ldots, n) \in \operatorname{ch} Q .
\end{aligned}
$$

If $j=p+1$, then

$$
\begin{aligned}
& \mathbf{i} * \mathbf{j}=(i, \ldots, n, n, \ldots, p+1, p, p+1, \ldots, n) \in \operatorname{ch} Q, \\
& \stackrel{(4.2),(4.4)}{\Longrightarrow}(i, \ldots, n, n, \ldots, p, p+1, p+1, \ldots, n) \in \operatorname{ch} Q, \\
& \stackrel{(4.3)}{\Rightarrow}(i, \ldots, p, p+1, p, \ldots, n, n, \ldots, p+1, p+1, \ldots, n) \in \operatorname{ch} Q, \\
& \stackrel{(4.2),(4.4)}{\Longrightarrow}(i, \ldots, p, p, p+1, \ldots, n, n, \ldots, p+1, p+1, \ldots, n) \in \operatorname{ch} Q .
\end{aligned}
$$

If $j=p$, then

$$
\begin{array}{rl}
\mathbf{i} & * \mathbf{j}=(i, \ldots, n, n, \ldots, p+1, p, p, p+1 \ldots, n) \in \operatorname{ch} Q, \\
& \stackrel{(4.4)}{\Rightarrow}(i, \ldots, n, n, \ldots, p, p+1, p, p+1, \ldots, n) \in \operatorname{ch} Q, \\
& \stackrel{(4.3)}{\Rightarrow}(i, \ldots, p, p+1, p, \ldots, n, n, \ldots, p, p+1, \ldots, n) \in \operatorname{ch} Q, \\
& \stackrel{(4.2),(4.4)}{\Longrightarrow}(i, \ldots, p, p, p+1, \ldots, n, n, \ldots, p, p+1, \ldots, n) \in \operatorname{ch} Q .
\end{array}
$$


If $j<p$, then

$$
\begin{aligned}
& \mathbf{i} * \mathbf{j}=(i, \ldots, n, n, \ldots, j, p, p+1 \ldots, n) \in \operatorname{ch} Q, \\
& \stackrel{(4.4)}{\Rightarrow}(i, \ldots, n, n, \ldots, p+1, p, p-1, p, \ldots, j, p+1, \ldots, n) \in \operatorname{ch} Q, \\
& \stackrel{(4.2),(4.4)}{\Longrightarrow}(i, \ldots, n, n, \ldots, p+1, p, p, p-1, \ldots, j, p+1, \ldots, n) \in \operatorname{ch} Q, \\
& \stackrel{(4.4)}{\Rightarrow}(i, \ldots, n, n, \ldots, p, p+1, p, p-1, \ldots, j, p+1, \ldots, n) \in \operatorname{ch} Q, \\
& \stackrel{(4.3)}{\Rightarrow}(i, \ldots, p, p+1, p, \ldots, n, n, \ldots,, j, p+1, \ldots, n) \in \operatorname{ch} Q \\
& \stackrel{(4.2),(4.4)}{\Longrightarrow}(i, \ldots, p, p, p+1, \ldots, n, n, \ldots, j, p+1, \ldots, n) \in \operatorname{ch} Q .
\end{aligned}
$$

Applying this argument repeatedly, we determine that

$$
\begin{gathered}
(i, \ldots, p, p, \ldots, n-2, n-2, n-1, n, n, \ldots, j, n-1, n) \in \operatorname{ch} Q \\
\stackrel{(4.2),(4.3),(4.4)}{\Longrightarrow}(i, \ldots, p, p, \ldots, n-1, n, n, n-1, n-1, \ldots, j, n) \in \operatorname{ch} Q \\
\stackrel{(4.2),(4.4)}{\Longrightarrow}(i, \ldots, p, p, \ldots, n-1, n, n-1, n, n-1, \ldots, j, n) \in \operatorname{ch} Q \\
\stackrel{(4.2),(4.4)}{\Longrightarrow}(i, \ldots, p, p, \ldots, n-1, n-1, n, n, n-1, \ldots, j, n) \in \operatorname{ch} Q \\
\quad \stackrel{(4.3)}{\Rightarrow}(i, \ldots, p, p, \ldots, n-1, n-1, n, n, n-1, n, \ldots, j) \in \operatorname{ch} Q \\
(4.2),(4.5) \\
\stackrel{\mathrm{g}}{\Longrightarrow}(i, \ldots, p, p, \ldots, n-1, n-1, n, n, n, n-1, \ldots, j) \in \operatorname{ch} Q .
\end{gathered}
$$

We suppose that $p=n-1, n$. Then, the same argument as above gives

$$
\mathbf{k}=(i, \ldots, n-1, n-1, n, n, n, n-1, \ldots, j) \in \operatorname{ch} Q .
$$

Now for any nonzero submodule $L$ of $M, \operatorname{ch} L$ contains $\mathbf{j} * \mathbf{i}$ by Lemma 1.5 and (3.4). If $a=c$, then

$$
\begin{gathered}
\mathbf{j} * \mathbf{i}=(i, i+1, \ldots, n, i, \ldots, n, n, \ldots, j) \in \mathrm{ch} L, \\
\stackrel{(4.3)}{\Longrightarrow}(i, i+1, i, \ldots, n, i+1, \ldots, n, n, \ldots, j) \in \operatorname{ch} L, \\
\stackrel{(4.2),(4.4)}{\Longrightarrow}(i, i, i+1, \ldots, n, i+1, \ldots, n, n, \ldots, j) \in \operatorname{ch} L, \\
\vdots \\
\stackrel{(4.2),(4.4)}{\Longrightarrow}(i, i, i+1, i+1, \ldots, n-2, n-2, n-1, n, n-1, n, n, \ldots, j) \in \operatorname{ch} L, \\
\stackrel{(4.2),(4.4)}{\Longrightarrow} \mathbf{k}=(i, i, i+1, i+1, \ldots, n-2, n-2, n-1, n-1, n, n, n, \ldots, j) \in \operatorname{ch} L .
\end{gathered}
$$


If $a \succ c$, then

$$
\begin{gathered}
\mathbf{j} * \mathbf{i}=(p, \ldots, n-1, n, i, \ldots, n, n, \ldots, j) \in \mathrm{ch} L, \\
\stackrel{(4.3)}{\Rightarrow}(p, \ldots, n-1, i, \ldots, n, n-1, n, n, \ldots, j) \in \operatorname{ch} L, \\
\stackrel{(4.2),(4.5)}{\Longrightarrow}(p, \ldots, n-1, i, \ldots, n-1, n, n, n, \ldots, j) \in \operatorname{ch} L, \\
\vdots \\
\stackrel{(4.2),(4.4)}{\Longrightarrow} \mathbf{k}=(i, \ldots, p, p, p+1, p+1, \ldots, n-1, n-1, n, n, n, \ldots, j) \in \operatorname{ch} L .
\end{gathered}
$$

Hence, $\operatorname{ch} L$ contains $\mathbf{k}$ in either event. From the structure of $\mathbf{k}$ we see that it occurs in $\operatorname{ch} Q$ (resp. ch $L$ ) with multiplicity $\xi_{Q} \geq 2^{l-1} 3$ ! (resp. $\xi_{L} \geq 2^{l-1} 3$ !). Therefore, since $\xi_{M}=2 \times 2^{l-1} 3$, we have $\xi_{M}=\xi_{Q}=\xi_{L}$, which implies the result for type $\mathrm{B}_{n}$.

Now assume that $\mathfrak{A}$ is of type $\boldsymbol{C}_{n}$. If either (i) or (ii) holds, the proof is identical to that for type $\mathrm{A}_{n}$. We assume that (iii) holds. Write $\mathbf{i}=\mathbf{i}(a, b)=(1,2, \ldots, n, \ldots, j), \mathbf{j}=\mathbf{i}(c, d)=$ $(n, n-1, \ldots, q)$. Then

$$
\mathbf{k}:=(1,2, \ldots, n-1, n, n, n-1, n-1, \ldots, q, q, \ldots, j) .
$$

is a shuffle of $\mathbf{i}$ and $\mathbf{j}$. Let $l$ be the length of $\mathbf{j}$. Then (3.4) and (4.1) imply that the term $\mathbf{k}$ appears in ch $M$ with multiplicity $\xi_{M}=2^{l}$.

For any nonzero quotient $Q$ of $M, \operatorname{ch} Q$ contains $\mathbf{i} * \mathbf{j}$ by (3.4) and Lemma 1.5, We claim that $\mathbf{k}$ occurs in $\operatorname{ch} Q$ as a term. Note that $a_{n-1, n}=-2$. The quantum Serre relations (1.3) and (4.2) imply

$$
\begin{aligned}
& \mathbf{i} * \mathbf{j}=(1, \ldots, n, \ldots, j, n, \ldots, q) \in \operatorname{ch} Q, \\
& \stackrel{(4.3)}{\Rightarrow}(1, \ldots, n-1, n, n-1, n, \ldots, j, n-1, \ldots, q) \in \operatorname{ch} Q, \\
& \stackrel{(4.2),(4.4)}{\Longrightarrow}(1, \ldots, n-1, n, n, n-1, \ldots, j, n-1, \ldots, q) \in \operatorname{ch} Q .
\end{aligned}
$$

Continuing this reasoning gives

$$
\mathbf{k}=(1, \ldots, n, n, \ldots, q, q, \ldots, j) \in \operatorname{ch} Q .
$$

For any nonzero submodule $L$ of $M, \operatorname{ch} L$ contains $\mathbf{j} * \mathbf{i}$ by Lemma 1.5 and (3.4). Then, using a similar argument, we have

$$
\begin{gathered}
\mathbf{j} * \mathbf{i}=(n, \ldots, q, 1,2, \ldots, n, \ldots, j) \in \operatorname{ch} L, \\
\stackrel{(4.2),(4.3),(4.4)}{\Longrightarrow}(n, n-1,1, \ldots, n-1, n, n-1, \ldots, q, q, \ldots, j) \in \operatorname{ch} L, \\
\stackrel{(4.2),(4.3),(4.4)}{\Longrightarrow}(n, 1, \ldots, n-2, n-1, n-1, n, n-1, \ldots, q, q, \ldots, j) \in \operatorname{ch} L,
\end{gathered}
$$




$$
\begin{aligned}
& \stackrel{(4.2),(4.5)}{\Longrightarrow}(n, 1, \ldots, n-2, n-1, n, n-1, n-1, \ldots, q, q, \ldots, j) \in \operatorname{ch} L, \\
& \stackrel{(4.3)}{\Rightarrow}(1, \ldots, n, n-1, n, n-1, n-1, \ldots, q, q, \ldots, j) \in \operatorname{ch} L, \\
& \stackrel{(4.2),(4.4)}{\Longrightarrow} \mathbf{k}=(1, \ldots, n, n, \ldots, q, q, \ldots, j) \in \operatorname{ch} L .
\end{aligned}
$$

As $\mathbf{k}$ occurs in $\operatorname{ch} Q$ (resp. ch $L$ ) with multiplicity $\xi_{Q} \geq 2^{l}$ (resp. $\xi_{L} \geq 2^{l}$ ), and since $\xi_{M}=2^{l}$, the assertion follows from $\xi_{M}=\xi_{Q}=\xi_{L}$.

Lastly, we assume that $\mathfrak{A}$ is of type $\mathrm{D}_{n}$. If (i) holds, then the proof is identical to that for type $\mathrm{A}_{n}$.

Suppose that (ii) holds. Write $\mathbf{i}=\mathbf{i}(a, b)=(n, n-1, n-2, \ldots, j), \mathbf{j}=\mathbf{i}(c, d)=(n, n-$ $1, n-2, \ldots, q)$. Then

$$
\mathbf{k}:=(n, n, n-1, n-1, n-2, n-2, \ldots, q, q, \ldots, j) .
$$

is a shuffle of $\mathbf{i}$ and $\mathbf{j}$. Let $l$ be the length of $\mathbf{j}$. The equations (3.4) and (4.1) imply that the term $\mathbf{k}$ appears in ch $M$ with multiplicity $\xi_{M}=2^{l}$.

By Lemma 1.5 and (3.4), $\mathbf{i} * \mathbf{j}$ appears in the character ch $Q$ of any quotient module $Q \subseteq M$. We will show that $\mathbf{k}$ also appears in $\operatorname{ch} Q$. Note that $a_{n, n-2}=a_{n-2, n}=a_{n-1, n-2}=a_{n-2, n-1}=$ -1 and $a_{n-1, n}=a_{n, n-1}=0$. The quantum Serre relations (1.3) imply

$$
\begin{array}{r}
\mathbf{i} * \mathbf{j}=(n, n-1, n-2 \ldots, j, n, n-1, n-2, \ldots, q) \in \operatorname{ch} Q, \\
\stackrel{(4.3)}{\Rightarrow}(n-1, n, n-2, n, \ldots, j, n-1, n-2, \ldots, q) \in \operatorname{ch} Q, \\
\stackrel{(4.2),(4.3),(4.4)}{\Longrightarrow}(n, n, n-1, n-2, \ldots, j, n-1, n-2, \ldots, q) \in \operatorname{ch} Q, \\
\stackrel{(4.2),(4.4)}{\Longrightarrow}(n, n, n-1, n-2, n-1 \ldots, j, n-2, \ldots, q) \in \operatorname{ch} Q, \\
\stackrel{(4.2),(4.5)}{\Longrightarrow}(n, n, n-1, n-1, n-2 \ldots, j, n-2, \ldots, q) \in \operatorname{ch} Q .
\end{array}
$$

Continuing this reasoning gives

$$
\mathbf{k}=(n, n, n-1, n-1, n-2, n-2, \ldots, q, q, \ldots, j) \in \operatorname{ch} Q .
$$

For any nonzero submodule $L$, since $\operatorname{ch} L$ contains $\mathbf{j} * \mathbf{i}$ by Lemma 1.5, the same argument shows that $\operatorname{ch} L$ contains $\mathbf{k}$. Since $\mathbf{k}$ occurs in $\operatorname{ch} Q(\operatorname{resp} \cdot \operatorname{ch} L)$ with multiplicity $\xi_{Q} \geq 2^{l}$ (resp. $\xi_{L} \geq 2^{l}$ ), the assertion follows from $\xi_{M}=\xi_{Q}=\xi_{L}$.

We now assume that (iii) holds. Since the proof is identical to that for type $A_{n}$ if $b=\bar{n}$ or $b=n$, we may assume that $n-1 \succeq b$. Write $\mathbf{i}=\mathbf{i}(a, b)=(1, \ldots, n-2, n, n-1, n-2, \ldots, j)$, $\mathbf{j}=\mathbf{i}(c, d)=(n, n-1, n-2, \ldots, q)$ and

$$
\mathbf{k}:=(1, \ldots, n-2, n, n, n-1, n-1, n-2, n-2, \ldots, q, q, \ldots, j) .
$$


Let $l$ be the length of $\mathbf{j}$. Then $\mathbf{k}$ is a shuffle of $\mathbf{i}$ and $\mathbf{j}$ and appears in $\operatorname{ch} M$ with multiplicity $\xi_{M}=2^{l}$.

For any nonzero quotient $Q$ of $M, \operatorname{ch} Q$ contains $\mathbf{i} * \mathbf{j}$ by (3.4) and Lemma 1.5. Using the same argument as in case (ii), we obtain

$$
\mathbf{k}=(1, \ldots, n-2, n, n, n-1, n-1, n-2 \ldots, j, n-2, \ldots, q) \in \operatorname{ch} Q .
$$

For any nonzero submodule $L$ of $M, \operatorname{ch} L$ contains $\mathbf{j} * \mathbf{i}$ by Lemma 1.5 and (3.4). By the same argument as in the $C_{n}$ case, we have

$$
\begin{gathered}
\mathbf{j} * \mathbf{i}=(n, \ldots, q-1, q, 1, \ldots, n-2, n, n-1, n-2 \ldots, j) \in \operatorname{ch} L, \\
\stackrel{(4.2),(4.3),(4.4)}{\Longrightarrow}(n, n-1, n-2,1, \ldots, n-2, n, n-1, n-2, \ldots, q, q, \ldots, j) \in \operatorname{ch} L, \\
\stackrel{(4.2),(4.4)}{\Longrightarrow}(n, n-1,1, \ldots, n-2, n-2, n, n-1, n-2, \ldots, q, q, \ldots, j) \in \operatorname{ch} L, \\
\stackrel{(4.4)}{\Rightarrow}(n, n-1,1, \ldots, n-2, n, n-2, n-1, n-2, \ldots, q, q, \ldots, j) \in \operatorname{ch} L, \\
\stackrel{(4.2),(4.4)}{\Longrightarrow}(n, n-1,1, \ldots, n-2, n, n-1, n-2, n-2, \ldots, q, q, \ldots, j) \in \operatorname{ch} L, \\
\stackrel{(4.3)}{\Rightarrow}(n, 1, \ldots, n-1, n-2, n-1, n, n-2, n-2, \ldots, q, q, \ldots, j) \in \operatorname{ch} L, \\
\stackrel{(4.2),(4.4)}{\Longrightarrow}(n, 1, \ldots, n-2, n, n-1, n-1, n-2, n-2, \ldots, q, q, \ldots, j) \in \operatorname{ch} L, \\
\stackrel{(4.3)}{\Rightarrow}(1, \ldots, n, n-2, n, n-1, n-1, n-2, n-2, \ldots, q, q, \ldots, j) \in \operatorname{ch} L, \\
\stackrel{(4.2),(4.4)}{\Longrightarrow} \mathbf{k}=(1, \ldots, n-2, n, n, n-1, n-1, n-2, n-2, \ldots, q, q, \ldots, j) \in \operatorname{ch} L .
\end{gathered}
$$

Since the sequence $\mathbf{k}$ occurs in $\operatorname{ch} Q($ resp. $\operatorname{ch} L)$ with multiplicity $\xi_{Q} \geq 2^{l}\left(\right.$ resp. $\left.\xi_{L} \geq 2^{l}\right)$, by $\xi_{M}=2^{l}$, the assertion follows from $\xi_{M}=\xi_{Q}=\xi_{L}$.

For $a, b \in \mathbf{B}$, we define

$$
\delta(a \succeq b)= \begin{cases}1 & \text { if } a \succeq b, \\ 0 & \text { otherwise }\end{cases}
$$

In the $\mathrm{A}_{n}$ case, it follows from [15, Lem. 4.3] that, for $d_{k} \in \mathbb{Z}_{\geq 0}$ and $b_{k} \in \mathbf{B}$ with $b_{1} \succ b_{2} \succ$ $\cdots \succ b_{m}$

$$
\tilde{f}_{1}^{t_{1}} \tilde{f}_{2}^{t_{2}} \cdots \tilde{f}_{n}^{t_{n}} \mathbf{1} \simeq \operatorname{lnd}\left(\underset{k=1}{\mathrm{\otimes}} \Delta_{\left(\overline{1}, b_{k}\right)}^{\otimes d_{k}}\right)
$$

where $t_{i}=\sum_{j=1}^{m} d_{j} \delta\left(\bar{\imath} \succ b_{j}\right)$ for $i=1, \ldots, n$. Next we will extend this result to the other finite classical types. We first divide the crystal $\mathbf{B}$ for types $\mathrm{B}_{n}, \mathrm{C}_{n}, \mathrm{D}_{n}$ into two pieces so that each part is almost the same as the crystal or the dual of the crystal for type A. For type $\mathrm{B}_{n}$ (resp. $\mathrm{C}_{n}, \mathrm{D}_{n}$ ), the crystal $\mathbf{B}$ is cut at the element 0 (resp. $\bar{n}, \overline{n-1}$ ). In the next result we give an analogue of (4.8) for each part. 
Lemma 4.2. Let $b_{k} \in \mathbf{B}$, and $d_{k} \in \mathbb{Z}_{\geq 0}$ for $k=1, \ldots, m$. Set

$$
b_{\bullet}= \begin{cases}\overline{n+1} & \left(\mathrm{~A}_{n}\right), \\ 0 & \left(\mathrm{~B}_{n}\right), \\ \bar{n} & \left(\mathrm{C}_{n}\right), \\ \overline{n-1} & \left(\mathrm{D}_{n}\right) .\end{cases}
$$

(1) If $b_{1} \succ b_{2} \succ \cdots \succ b_{m} \succeq b_{\bullet}$, then

$$
\tilde{f}_{1}^{t_{1}} \tilde{f}_{2}^{t_{2}} \cdots \tilde{f}_{l}^{t_{l}} \mathbf{1} \simeq \operatorname{lnd}\left(\underset{k=1}{\otimes} \Delta_{\left(\overline{1}, b_{k}\right)}^{\otimes d_{k}}\right)
$$

where $l=n \quad\left(\mathrm{~A}_{n}, \mathrm{~B}_{n}\right), \quad l=n-1 \quad\left(\mathrm{C}_{n}\right), \quad l=n-2 \quad\left(\mathrm{D}_{n}\right)$ and $t_{i}:=\sum_{j=1}^{m} d_{j} \delta\left(\bar{\imath} \succ b_{j}\right)$ for $i=1, \ldots, l$.

(2) Let

$$
t_{i}= \begin{cases}\sum_{j=1}^{m} d_{j} \delta\left(i \succeq b_{j}\right) & \text { if } i=1, \ldots, n\left(\mathrm{~B}_{n}, \mathrm{C}_{n}\right), \quad i=1, \ldots, n-2\left(\mathrm{D}_{n}\right), \\ \sum_{j=1}^{m} d_{j} \delta\left(\bar{n} \succeq b_{j}\right) & \text { if } i=n-1\left(\mathrm{D}_{n}\right), \\ \sum_{j=1}^{m} d_{j} \delta\left(n \succeq b_{j}\right) & \text { if } i=n\left(\mathrm{D}_{n}\right) .\end{cases}
$$

Then we have

(i) if $b_{\bullet} \succ b_{1} \succ b_{2} \succ \cdots \succ b_{m}$ for type $\mathrm{B}_{n}$, then

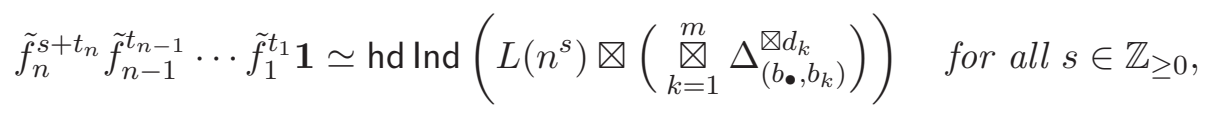

and the head occurs with multiplicity one as a composition factor of

$$
\operatorname{lnd}\left(L\left(n^{s}\right) \otimes\left(\underset{k=1}{\otimes} \Delta_{\left(b_{\bullet}, b_{k}\right)}^{\otimes d_{k}}\right)\right),
$$

(ii) if $b_{\bullet} \succ b_{m} \succ b_{m-1} \succ \cdots \succ b_{1}$ for type $\mathrm{C}_{n}$, $\mathrm{D}_{n}$, then

$$
\tilde{f}_{n}^{t_{n}} \cdots \tilde{f}_{2}^{t_{2}} \tilde{f}_{1}^{t_{1}} \mathbf{1} \simeq \operatorname{lnd}\left(\underset{k=1}{\bigotimes} \Delta_{\left(b_{\bullet}, b_{k}\right)}^{\otimes d_{k}}\right) .
$$

Proof. For $k_{1}, \ldots, k_{n} \in \mathbb{Z}_{\geq 0}$, it follows from Lemma 1.8 that

$$
\begin{aligned}
\tilde{f}_{1}^{k_{1}} \tilde{f}_{2}^{k_{2}} \cdots \tilde{f}_{n}^{k_{n}} \mathbf{1} & \simeq \text { hd Ind }\left(L\left(1^{k_{1}}\right) \otimes \cdots \otimes L\left(n^{k_{n}}\right)\right), \\
\tilde{f}_{n}^{k_{n}} \tilde{f}_{n-1}^{k_{n-1}} \cdots \tilde{f}_{1}^{k_{1}} \mathbf{1} & \simeq \text { hd Ind }\left(L\left(n^{k_{n}}\right) \otimes \cdots \otimes L\left(1^{k_{1}}\right)\right) .
\end{aligned}
$$

(1) When $\mathfrak{A}$ is of type $\mathrm{A}_{n}, \mathrm{C}_{n}, \mathrm{D}_{n}$, this can be shown in the same way as for [15, Lem. 4.3]. Suppose that $\mathfrak{A}$ is of type $\mathrm{B}_{n}$. Note that $\Delta_{\left(\overline{1}, b_{k}\right)}$ is one of the cuspidal representations given in [7, Sec. 6.2] and [21, Sec. 8.5]. By [21, Lem. 6.6], we have $N_{k}:=\operatorname{lnd} \Delta_{\left(\overline{1}, b_{k}\right)}^{\square d_{k}}$ is irreducible for $k=1, \ldots, m$. We write $\mathbf{i}\left(\overline{1}, b_{k}\right)=\left(1,2, \ldots, l_{k}\right)$ and let

$$
\mathbf{k}_{k}=(\underbrace{1, \ldots, 1}_{d_{k}}, \underbrace{2, \ldots, 2}_{d_{k}}, \ldots, \underbrace{l_{k}, \ldots, l_{k}}_{d_{k}})
$$


for $k=1, \ldots, m$. Then $\mathbf{k}_{k}$ appears in $\operatorname{ch} N_{k}$ with multiplicity $\left(d_{k} !\right)^{l_{k}}$. Since $b_{1} \succ b_{2} \succ \cdots \succ$ $b_{m}$, the sequence $\mathbf{k}=\mathbf{k}_{m} * \cdots * \mathbf{k}_{1}$ appears in $\operatorname{ch}\left(\operatorname{Ind}\left(N_{m} \otimes \cdots \otimes N_{1}\right)\right)$ with multiplicity $\left(d_{m} !\right)^{l_{m}} \cdots\left(d_{1} !\right)^{l_{1}}$. Lemma 1.7 and Lemma 4.1 imply that $\operatorname{Ind}\left(\underset{k=1}{\mathrm{\otimes}} N_{k}\right) \simeq \operatorname{Ind}\left(N_{m} \otimes \cdots \otimes N_{1}\right)$ is irreducible.

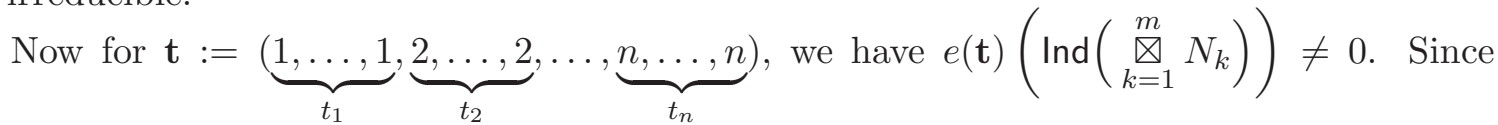
Ind $\left(\underset{k=1}{\bigotimes} N_{k}\right)$ is irreducible, it follows from Lemma 1.5 that there is a surjective homomorphism

$$
\operatorname{lnd}\left(L\left(1^{t_{1}}\right) \otimes \cdots \otimes L\left(n^{t_{n}}\right)\right) \rightarrow \operatorname{Ind}\left(\underset{k=1}{\bigotimes_{k}^{m}} N_{k}\right) .
$$

Therefore, $\tilde{f}_{1}^{t_{1}} \cdots \tilde{f}_{n}^{t_{n}} \mathbf{1} \simeq \operatorname{lnd}\left(\underset{k=1}{\bigotimes_{k}} N_{k}\right)$ by (4.9) .

(2) For this part, note that since $\Delta_{\left(b_{\bullet}, b_{k}\right)}$ is a cuspidal representation as in [7, Sec. 6] and [21, Sec. 8], $N_{k}:=\operatorname{Ind} \Delta_{\left(b_{\bullet}, b_{k}\right)}^{\otimes d_{k}}$ is irreducible by [21, Lem. 6.6]. We now separate considerations according to the type.

$\left(\right.$ Case $\left.\mathrm{B}_{n}\right)$ When $\mathfrak{A}$ is of type $\mathrm{B}_{n}$, let $N=\operatorname{lnd}\left(\Delta_{\left(n, b_{m}\right)}^{\nabla d_{m}} \otimes \cdots \otimes \Delta_{\left(n, b_{1}\right)}^{\nabla d_{1}}\right)$. By [15, Lem. 4.3], we have $\tilde{f}_{n-1}^{t_{n-1}} \cdots \tilde{f}_{1}^{t_{1}} \mathbf{1} \simeq N$. By (4.6) and Lemma 4.1, for $d \in \mathbb{Z}_{\geq 0}$ we obtain

$$
\begin{aligned}
& \text { Ind }\left(L\left(n^{d}\right) \otimes \Delta_{\left(n, b_{k}\right)}^{\otimes d}\right) \simeq \operatorname{lnd}\left(L\left(n^{d-1}\right) \otimes L(n) \otimes \Delta_{\left(n, b_{k}\right)} \otimes \Delta_{\left(n, b_{k}\right)}^{\otimes d-1}\right) \\
& \rightarrow \text { Ind }\left(L\left(n^{d-1}\right) \otimes \Delta_{\left(0, b_{k}\right)} \otimes \Delta_{\left(n, b_{k}\right)}^{\otimes d-1}\right) \simeq \operatorname{lnd}\left(L\left(n^{d-1}\right) \otimes \Delta_{\left(n, b_{k}\right)}^{\otimes d-1} \otimes \Delta_{\left(0, b_{k}\right)}\right) \\
& \quad \vdots \\
& \rightarrow \operatorname{lnd}\left(\Delta_{\left(0, b_{k}\right)}^{\otimes d}\right) .
\end{aligned}
$$

Since $t_{n}=d_{1}+\cdots+d_{m}$, by the same argument as above, we have the following chain of surjective homomorphisms

$$
\begin{aligned}
& \text { Ind }\left(L\left(n^{s+t_{n}}\right) \otimes N\right) \rightarrow \operatorname{lnd}\left(L\left(n^{s+t_{n}-d_{m}}\right) \otimes \Delta_{\left(b_{\bullet}, b_{m}\right)}^{\otimes d_{m}} \otimes \Delta_{\left(n, b_{m-1}\right)}^{\otimes d_{m-1}} \otimes \cdots \otimes \Delta_{\left(n, b_{1}\right)}^{\otimes d_{1}}\right) \\
& \simeq \operatorname{lnd}\left(L\left(n^{s+t_{n}-d_{m}}\right) \otimes \Delta_{\left(n, b_{m-1}\right)}^{\otimes d_{m-1}} \otimes \cdots \otimes \Delta_{\left(n, b_{1}\right)}^{\otimes d_{1}} \otimes \Delta_{\left(b_{\bullet}, b_{m}\right)}^{\otimes d_{m}}\right) \\
& \rightarrow \operatorname{Ind}\left(L\left(n^{s+t_{n}-d_{m}-d_{m-1}}\right) \otimes \Delta_{\left(n, b_{m-2}\right)}^{\bigotimes d_{m-2}} \otimes \cdots \otimes \Delta_{\left(n, b_{1}\right)}^{\otimes d_{1}} \otimes \Delta_{\left(b_{\bullet}, b_{m-1}\right)}^{\bigotimes d_{m-1}} \otimes \Delta_{\left(b_{\bullet}, b_{m}\right)}^{\otimes d_{m}}\right)
\end{aligned}
$$

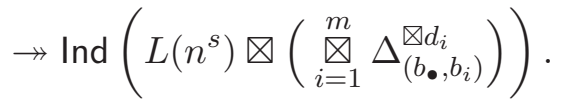

Therefore, by Lemma 1.8 and [19, Lem. 3.13], we obtain

$$
\tilde{f}_{n}^{s+t_{n}} \tilde{f}_{n-1}^{t_{n-1}} \cdots \tilde{f}_{1}^{t_{1}} \mathbf{1} \simeq \operatorname{hd} \operatorname{lnd}\left(L\left(n^{s+t_{n}}\right) \otimes N\right) \simeq \operatorname{hd} \operatorname{lnd}\left(L ( n ^ { s } ) \otimes \left({\left.\left.\underset{i=1}{\bigotimes} \Delta_{\left(b_{\bullet}, b_{i}\right)}^{\otimes d_{i}}\right)\right)}^{m}\right.\right.
$$


and this module has multiplicity one as a composition factor of $\operatorname{lnd}\left(L\left(n^{s}\right) \otimes\left(\underset{i=1}{\otimes} \Delta_{\left(b_{\bullet}, b_{i}\right)}^{\otimes d_{i}}\right)\right)$.

$\left(\right.$ Case $\left.C_{n}\right)$ For type $C_{n}$, we write $\mathbf{i}\left(b_{\bullet}, b_{j}\right)=\left(n, n-1, \ldots, n-l_{j}+1\right)$ and let

$$
\mathbf{k}_{k}=(\underbrace{n, \ldots, n}_{d_{k}}, \underbrace{n-1, \ldots, n-1}_{d_{k}}, \ldots, \underbrace{n-l_{k}+1, \ldots, n-l_{k}+1}_{d_{k}})
$$

for $k=1, \ldots, m$. Then $\mathbf{k}_{k}$ appears in $\operatorname{ch} N_{k}$ with multiplicity $\left(d_{k} !\right)^{l_{k}}$. Since $b_{\bullet} \succ b_{m} \succ$ $b_{m-1} \succ \cdots \succ b_{1}$, the sequence $\mathbf{k}:=\mathbf{k}_{1} * \cdots * \mathbf{k}_{m}$ appears in ch $\operatorname{Ind}\left(\underset{k=1}{\bigotimes} N_{k}\right)$ with multiplicity $\left(d_{1} !\right)^{l_{1}} \cdots\left(d_{m} !\right)^{l_{m}}$. It follows from Lemma 1.7 and Lemma 4.1 that $\operatorname{Ind}\left(\underset{k=1}{m} N_{k}\right)$ is irreducible.

Let $\mathbf{t}=(\underbrace{n, \ldots, n}_{t_{n}}, \underbrace{n-1, \ldots, n-1}_{t_{n-1}}, \ldots, \underbrace{1, \ldots, 1}_{t_{1}})$. Then we have $e(\mathbf{t})\left(\operatorname{lnd}\left(\bigotimes_{k=1}^{m} N_{k}\right)\right) \neq 0$.

Since Ind $\left(\underset{k=1}{\bigotimes} N_{k}\right)$ is irreducible, by Lemma 1.5 there exists a surjective homomorphism

$$
\operatorname{lnd}\left(L\left(n^{t_{n}}\right) \otimes \cdots \otimes L\left(1^{t_{1}}\right)\right) \rightarrow \operatorname{lnd}\left(\underset{k=1}{\bigotimes_{k}} N_{k}\right)
$$

Therefore, it follows from (4.10) that $\tilde{f}_{n}^{t_{n}} \cdots \tilde{f}_{2}^{t_{2}} \tilde{f}_{1}^{t_{1}} \mathbf{1} \simeq \operatorname{lnd}\left(\underset{k=1}{\underset{m}{\otimes}} N_{k}\right)$.

(Case $\mathrm{D}_{n}$ ) For $k=1, \ldots, m$, let $l_{k}$ be the length of $\mathbf{i}\left(\frac{k=1}{n-1}, \bar{n}\right) * \mathbf{i}\left(\bar{n}, b_{k}\right)$ if $\bar{n} \succ b_{k}$, and $l_{k}=1$ if $b_{k}=n$ or $b_{k}=\bar{n}$. We will show that Ind $\left(\underset{k=1}{\otimes} \Delta_{\left(b_{\bullet}, b_{k}\right)}^{\otimes d_{k}}\right)$ is irreducible for type $\mathrm{D}_{n}$.

First suppose that $b_{m-1} \neq n-1$. Let

$$
\mathbf{k}_{k}= \begin{cases}\underbrace{(\underbrace{}_{k}-1, \ldots, n-1}_{d_{k}}) & \text { if } b_{k}=\bar{n}, \\ \underbrace{(\underbrace{}_{1} \ldots, n}_{d_{k}}, \underbrace{n-1, \ldots, n-1}_{d_{k}}, \ldots, \underbrace{n-l_{k}+1, \ldots, n-l_{k}+1}_{d_{k}}) & \text { otherwise. }\end{cases}
$$

for $k=1, \ldots, m$. Then $\mathbf{k}_{k}$ appears in $\operatorname{ch} N_{k}$ with multiplicity $\left(d_{k} !\right)^{l_{k}}$. Since $b_{m} \succ b_{m-1} \succ$ $\cdots \succ b_{1}$ and $b_{m-1} \neq n-1$, the sequence $\mathbf{k}:=\mathbf{k}_{1} * \cdots * \mathbf{k}_{m}$ appears in ch $\operatorname{lnd}\left(\underset{k=1}{\bigotimes} N_{k}\right)$ with multiplicity $\left(d_{1} !\right)^{l_{1}} \cdots\left(d_{m} !\right)^{l_{m}}$. It follows from Lemma 1.7 and Lemma 4.1 that $\operatorname{Ind}\left(\underset{k=1}{\bigotimes} N_{k}\right)$ is irreducible.

We now assume that $b_{m-1}=n-1$. Without loss of generality, we may suppose that $b_{m}=\bar{n}$. Since $\operatorname{lnd}(L(n-1) \otimes L(n)) \simeq \operatorname{lnd}(L(n) \otimes L(n-1))$, we have $\operatorname{lnd} \Delta_{(\overline{n-1, n-1)}}^{\otimes d_{m-1}} \otimes \Delta_{(\overline{n-1}, \bar{n})}^{\otimes d_{m}} \simeq \operatorname{lnd} \Delta_{(\overline{n-1}, \bar{n})}^{\otimes d_{m}} \otimes \Delta_{(\overline{n-1}, n-1)}^{\otimes d_{m-1}} \simeq \operatorname{lnd}\left(L\left(n^{d_{m-1}}\right) \otimes L\left((n-1)^{d_{m-1}+d_{m}}\right)\right)$

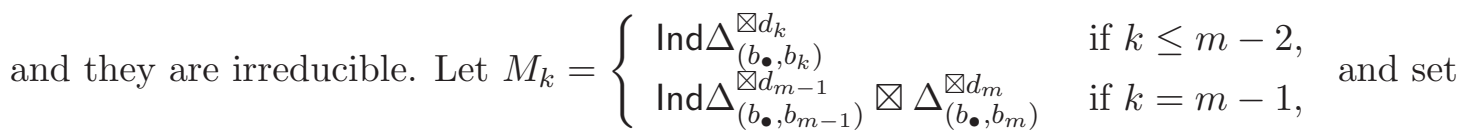




$$
\mathbf{k}_{k}= \begin{cases}(\underbrace{n, \ldots, n}_{d_{k}}, \underbrace{n-1, \ldots, n-1}_{d_{k}}, \ldots, \underbrace{n-\ell_{k}+1, \ldots, n-\ell_{k}+1}_{d_{k}}) & \text { if } k \leq m-2, \\ \underbrace{n, \ldots, n}_{d_{m-1}}, \underbrace{n-1, \ldots, n-1}_{d_{m-1}+d_{m}}) & \text { if } k=m-1 .\end{cases}
$$

Then the multiplicity of $\mathbf{k}_{k}$ in $\operatorname{ch} M_{k}$ is $\left(d_{k} !\right)^{l_{k}}$ if $k \leq m-2$ and $d_{m-1} !\left(d_{m}+d_{m-1}\right)$ ! if $k=m-1$. Since $b_{\bullet} \succ b_{m} \succ b_{m-1} \succ \cdots \succ b_{1}$, the sequence $\mathbf{k}:=\mathbf{k}_{1} * \cdots * \mathbf{k}_{m-1}$ appears in $\operatorname{ch}\left(\operatorname{Ind}\left(M_{1} \otimes \cdots \otimes M_{m-1}\right)\right)$ with multiplicity $\left(d_{1} !\right)^{l_{1}} \cdots\left(d_{m-2} !\right)^{l_{m-2}}\left(d_{m-1} !\right)\left(\left(d_{m}+d_{m-1}\right) !\right)$. It follows from Lemma 1.7 and Lemma 4.1 that $\operatorname{Ind}\left(M_{1} \otimes \cdots \otimes M_{m-1}\right)$ is irreducible.

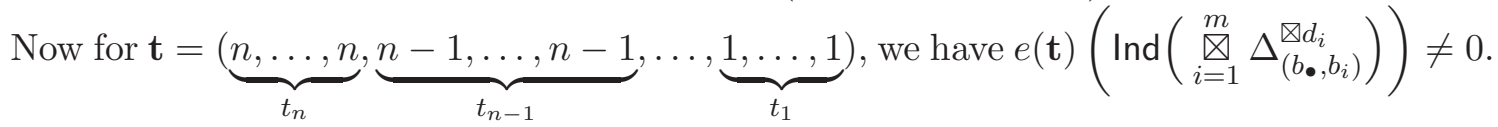
Since Ind $\left(\underset{i=1}{\otimes} \Delta_{\left(b_{\bullet}, b_{i}\right)}^{\square d_{i}}\right)$ is irreducible, Lemma 1.5 gives a surjective homomorphism

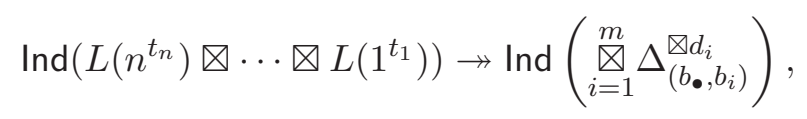

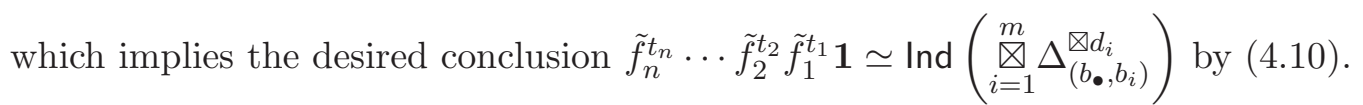

Recall the definition of $\widehat{\imath} \in \mathbf{B}$ from (3.2). Using the surjective homomorphism in (4.6), we can glue parts (1) and (2) of Lemma 4.2 together for types $B_{n}, C_{n}$, and $D_{n}$ to get the following result.

Lemma 4.3. Let $t_{i} \in \mathbb{Z}_{\geq 0}$ be such that

$$
\begin{array}{lr}
t_{1} \geq t_{2} \geq \cdots \geq t_{n^{\prime}-1} \geq t_{n^{\prime}} \geq t_{n^{\prime}+1}=0 & \left(\mathrm{~A}_{n}, \mathrm{C}_{n}\right), \\
2 t_{1} \geq 2 t_{2} \geq \cdots \geq 2 t_{n-1} \geq t_{n} \geq 2 t_{n+1} \geq \cdots \geq 2 t_{n^{\prime}-1} \geq t_{n^{\prime}}=0 & \left(\mathrm{~B}_{n}\right), \\
t_{1} \geq t_{2} \geq \cdots \geq t_{n-2} \geq t_{n-1}, t_{n} \geq t_{n+1} \geq \cdots \geq t_{n^{\prime}-1} \geq t_{n^{\prime}}=0 & \left(\mathrm{D}_{n}\right),
\end{array}
$$

where $n^{\prime}=n\left(\mathrm{~A}_{n}\right), n^{\prime}=2 n\left(\mathrm{~B}_{n}\right), n^{\prime}=2 n-1\left(\mathrm{C}_{n}, \mathrm{D}_{n}\right)$. Set

$$
\vartheta_{i}= \begin{cases}t_{i}-t_{i+1} & \text { if } i \leq n^{\prime} \quad\left(\mathrm{A}_{n}, \mathrm{C}_{n}\right), \quad i \leq n-2\left(\mathrm{~B}_{n}\right), i \leq n-3\left(\mathrm{D}_{n}\right), \\ t_{n-1}-\left\lceil\frac{t_{n}}{2}\right\rceil & \text { if } i=n-1 \quad\left(\mathrm{~B}_{n}\right), \\ \left\lceil\frac{t_{n}}{2}\right\rceil-\left\lfloor\frac{t_{n}}{2}\right\rfloor & \text { if } i=n \quad\left(\mathrm{~B}_{n}\right), \\ \left\lfloor\frac{t_{n}}{2}\right\rfloor-t_{n+1} & \text { if } i=n+1 \quad\left(\mathrm{~B}_{n}\right), \\ t_{n-2}-\max \left\{t_{n-1}, t_{n}\right\} & \text { if } i=n-2 \quad\left(\mathrm{D}_{n}\right), \\ \max \left\{0, t_{n}-t_{n-1}\right\} & \text { if } i=n-1 \quad\left(\mathrm{D}_{n}\right), \\ \max \left\{0, t_{n-1}-t_{n}\right\} & \text { if } i=n \quad\left(\mathrm{D}_{n}\right), \\ \min \left\{t_{n-1}, t_{n}\right\}-t_{n+1} & \text { if } i=n+1 \quad\left(\mathrm{D}_{n}\right), \\ t_{i-1}-t_{i} & \text { if } i \geq n+2 \quad\left(\mathrm{~B}_{n}, \mathrm{D}_{n}\right) .\end{cases}
$$


Then

(1) hd Ind $\left({\underset{i=1}{n^{\prime}} \Delta_{(\widehat{1}, i+1)}^{\otimes \vartheta_{i}}}_{i+1}\right)$ is irreducible and has multiplicity one as a composition factor of

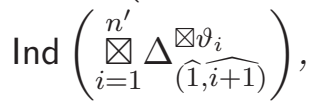

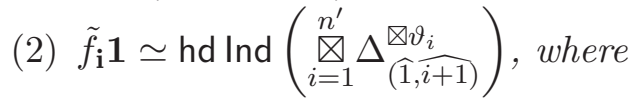

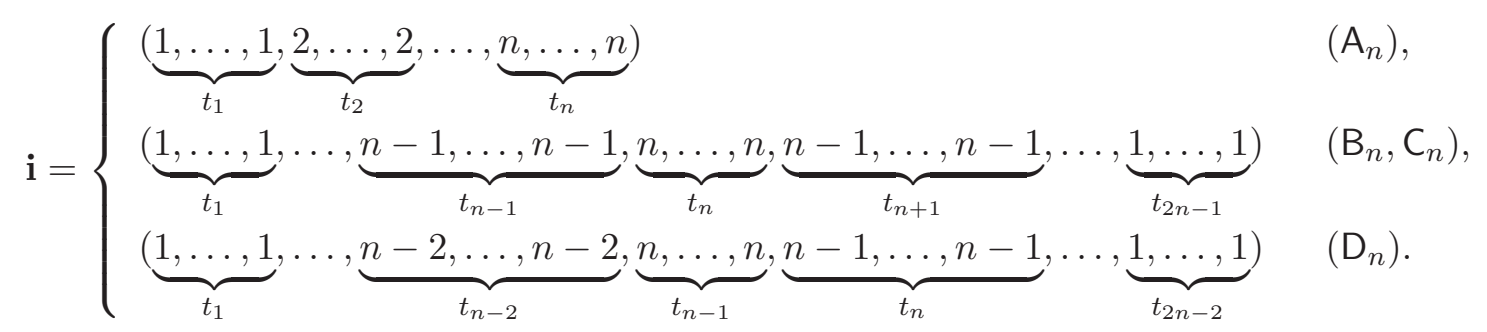

Proof. By Lemma 4.1 and (4.6), for $k \in \mathbb{Z}_{\geq 0}$ we have

$$
\begin{aligned}
& \operatorname{Ind}\left(\Delta_{(\widehat{1}, b)}^{\otimes k} \otimes \Delta_{(b, c)}^{\otimes k}\right) \simeq \operatorname{lnd}\left(\Delta_{(\widehat{1}, b)}^{\otimes k-1} \otimes \Delta_{(\widehat{1}, b)} \otimes \Delta_{(b, c)} \otimes \Delta_{(b, c)}^{\otimes k-1}\right) \\
& \rightarrow \text { Ind }\left(\Delta_{(\widehat{1}, b)}^{\otimes k-1} \otimes \Delta_{(\widehat{1}, c)} \otimes \Delta_{(b, c)}^{\otimes k-1}\right) \\
& \simeq \operatorname{Ind}\left(\Delta_{(\widehat{1}, b)}^{\otimes k-1} \otimes \Delta_{(b, c)}^{\otimes k-1} \otimes \Delta_{(\widehat{1}, c)}\right) \\
& \rightarrow \operatorname{Ind}\left(\Delta_{(\widehat{1}, c)}^{\otimes k}\right) \text {, }
\end{aligned}
$$

where $b \in B\left(\mathrm{~A}_{n}\right), \quad b=\widehat{n+1}\left(\mathrm{~B}_{n}\right), \quad b=\widehat{n}\left(\mathrm{C}_{n}\right), b=\widehat{n-1}\left(\mathrm{D}_{n}\right)$ and $c \in \mathbf{B}$ with $b \succ c$.

(Case $A_{n}$ ) The assertion in this case follows from Lemma 4.2.

$\left(\right.$ Case $\left.\mathrm{B}_{n}\right)$ For type $\mathrm{B}_{n}$, let

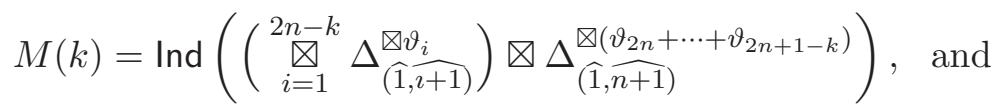

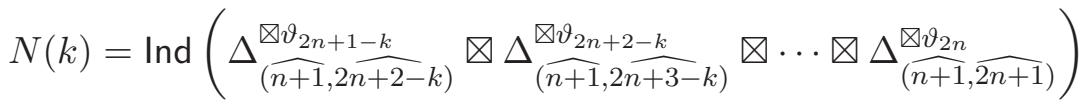

for $k=1, \ldots, n$. Note that $t_{n}=\left\lceil\frac{t_{n}}{2}\right\rceil+\left\lfloor\frac{t_{n}}{2}\right\rfloor,\left\lceil\frac{t_{n}}{2}\right\rceil=\vartheta_{n}+\cdots \vartheta_{2 n}$ and $\left\lfloor\frac{t_{n}}{2}\right\rfloor=\vartheta_{n+1}+\cdots+\vartheta_{2 n}$. If follows from Lemma 4.2 that

$$
M(n) \simeq \tilde{f}_{1}^{t_{1}} \cdots \tilde{f}_{n-1}^{t_{n-1}} \tilde{f}_{n}^{\left\lceil\frac{t_{n}}{2}\right\rceil} \mathbf{1}, \quad \operatorname{hd} N(n) \simeq \tilde{f}_{n}^{\left\lfloor\frac{t_{n}}{2}\right\rfloor} \tilde{f}_{n-1}^{t_{n+1}} \cdots \tilde{f}_{1}^{t_{2 n-1}} \mathbf{1} .
$$

By Lemma 4.2 and [19, Lem. 3.13], there is a surjective homomorphism

$$
\operatorname{lnd}\left(\Delta_{(\overline{1}, \bar{n})}^{\otimes\left\lceil\frac{t_{n}}{2}\right\rceil} \otimes L\left(n^{\left\lceil\frac{t_{n}}{2}\right\rceil}\right)\right) \rightarrow \operatorname{lnd} \Delta_{(\overline{1}, 0)}^{\otimes\left\lceil\frac{t_{n}}{2}\right\rceil},
$$


which yields the following surjective homomorphism

$$
\begin{aligned}
& \operatorname{lnd}\left(\left(\left(\underset{i=1}{\otimes-1} \Delta_{(\widehat{1}, \imath+1)}^{\otimes \vartheta_{i}}\right) \otimes \Delta_{(\widehat{1}, \widehat{n})}^{\otimes\left\lceil\frac{t_{n}}{2}\right\rceil}\right) \otimes\left(L\left(n^{\left\lceil\frac{t_{n}}{2}\right\rceil}\right) \otimes N(n)\right)\right) \\
& \rightarrow \operatorname{lnd}\left(\left(\underset{i=1}{n-1} \Delta_{(\widehat{1}, i+1)}^{\otimes \vartheta_{i}}\right) \otimes \Delta_{(\widehat{1}, n+1)}^{\otimes\left\lceil\frac{t_{n}}{2}\right\rceil} \otimes N(n)\right)
\end{aligned}
$$

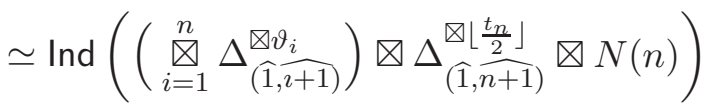

$$
\begin{aligned}
& \simeq \operatorname{lnd}(M(n) \otimes N(n)) \text {. }
\end{aligned}
$$

It follows from Lemma 4.2 that

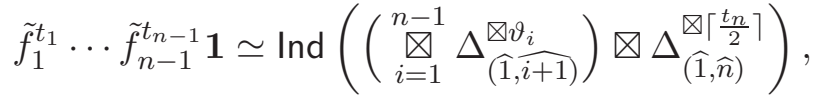

$$
\begin{aligned}
& \tilde{f}_{n}^{t_{n}} \tilde{f}_{n-1}^{t_{n+1}} \cdots \tilde{f}_{1}^{t_{2 n-1}} \mathbf{1} \simeq \text { hd Ind }\left(L\left(n^{\left\lceil\frac{t_{n}}{2}\right\rceil}\right) \otimes N(n)\right) \text {. }
\end{aligned}
$$

Since $\varepsilon_{i}\left(\operatorname{lnd}\left(L\left(n^{\left\lceil\frac{t_{n}}{2}\right\rceil}\right) \otimes N(n)\right)\right)=0$ for $i=1, \ldots, n-1$, by Lemma 1.8 we have

$$
\tilde{f}_{\mathrm{i}} \mathbf{1} \simeq \operatorname{hd} \operatorname{lnd}(M(n) \otimes N(n))
$$

and this module has multiplicity one as a composition factor of $\operatorname{lnd}(M(n) \otimes N(n))$.

Now by Lemma 4.1 and (4.11), we have the following chain of surjective homomorphisms

$$
\begin{aligned}
& \operatorname{lnd}(M(n) \otimes N(n))
\end{aligned}
$$

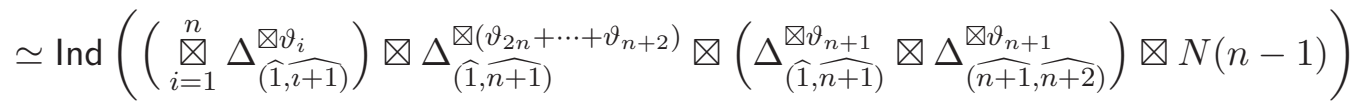

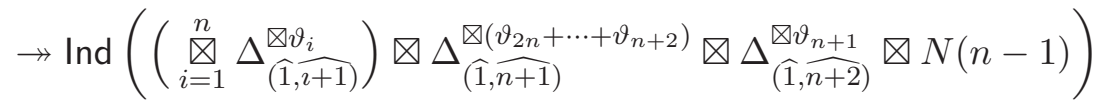

$$
\begin{aligned}
& \simeq \operatorname{lnd}(M(n-1) \otimes N(n-1))
\end{aligned}
$$

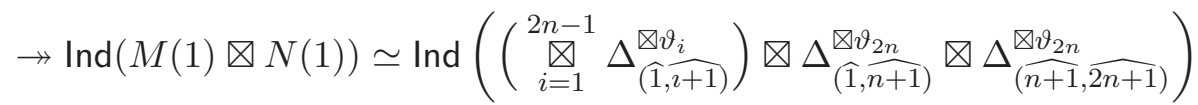

$$
\begin{aligned}
& \rightarrow \operatorname{Ind}\left(\underset{i=1}{\bigotimes_{i=1} \Delta_{(\widehat{1}, i+1)}^{\otimes \vartheta_{i}}}\right),
\end{aligned}
$$

which completes the proof for $\mathrm{B}_{n}$. 
(Case $C_{n}$ ) For type $C_{n}$, set

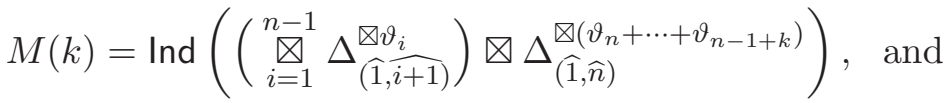

$$
\begin{aligned}
& N(k)=\operatorname{lnd}\left(\Delta_{(\widehat{n}, \hat{k+n})}^{\otimes \vartheta_{k+n-1}} \otimes \Delta_{(\widehat{n}, k+n-1)}^{\bigotimes \vartheta_{k+n-2}} \otimes \cdots \otimes \Delta_{(\widehat{n}, \widehat{n+1})}^{\otimes \vartheta_{n}}\right)
\end{aligned}
$$

for $1 \leq k \leq n$. It follows from Lemma 4.2 that

$$
M(n) \simeq \tilde{f}_{1}^{t_{1}} \tilde{f}_{2}^{t_{2}} \cdots \tilde{f}_{n-1}^{t_{n-1}} \mathbf{1}, \quad N(n) \simeq \tilde{f}_{n}^{t_{n}} \tilde{f}_{n-1}^{t_{n+1}} \cdots \tilde{f}_{1}^{t_{2 n-1}} \mathbf{1} .
$$

Since $\varepsilon_{i}(N(n))=0$ for $i=1, \ldots, n-1$, Lemma 1.8 implies

$$
\tilde{f}_{\mathbf{i}} \mathbf{1} \simeq \operatorname{hd} \operatorname{lnd}(M(n) \otimes N(n))
$$

and this module has multiplicity one as a composition factor in $\operatorname{lnd}(M(n) \otimes N(n))$.

By Lemma 4.1 and (4.11), we have a chain of surjective homomorphisms

$$
\begin{aligned}
& \operatorname{lnd}(M(n) \otimes N(n)) \simeq \operatorname{lnd}\left(M(n-1) \otimes \Delta_{(\widehat{1}, \widehat{n})}^{\bigotimes \vartheta_{2 n-1}} \otimes \Delta_{(\widehat{n}, 2 n)}^{\otimes \vartheta_{2 n-1}} \otimes N(n-1)\right) \\
& \rightarrow \text { Ind }\left(M(n-1) \otimes \Delta_{(\widehat{1}, 2 n)}^{\otimes \vartheta_{2 n-1}} \otimes N(n-1)\right) \\
& \simeq \operatorname{lnd}\left(M(n-1) \otimes N(n-1) \otimes \Delta_{(\widehat{1}, 2 n)}^{\otimes \vartheta_{2 n-1}}\right) \\
& \rightarrow \operatorname{lnd}\left(M(1) \otimes N(1) \otimes \Delta_{(\widehat{1}, \hat{n+2})}^{\otimes \vartheta_{n+1}} \otimes \cdots \otimes \Delta_{(\widehat{1}, 2 n)}^{\otimes \vartheta_{2 n-1}}\right) \\
& \rightarrow \operatorname{Ind}\left(\left(\underset{i=1}{n-1} \Delta_{(\widehat{1}, \imath+1)}^{\otimes \vartheta_{i}}\right) \otimes \Delta_{(\widehat{1}, n+1)}^{\otimes \vartheta_{n}} \otimes \cdots \otimes \Delta_{(\widehat{1}, 2 n)}^{\otimes \vartheta_{2 n-1}}\right) \\
& \simeq \operatorname{lnd}\left(\underset{i=1}{\bigotimes_{(1, i+1)}^{n-1}} \Delta_{(\widehat{1}, \hat{\nabla}}^{\otimes \vartheta_{i}}\right) \text {, }
\end{aligned}
$$

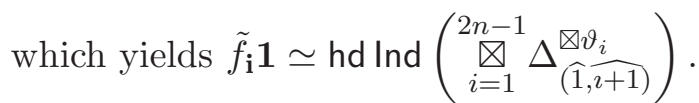

(Case $\mathrm{D}_{n}$ ) Without loss of generality, we may assume in the $\mathrm{D}_{n}$ case that $t_{n-1} \geq t_{n}$. Note that $\vartheta_{n-1}=0$ and $\vartheta_{n}=\max \left\{t_{n-1}, t_{n}\right\}-\min \left\{t_{n-1}, t_{n}\right\}$. Let

$$
\begin{aligned}
& M(k)=\operatorname{lnd}\left(\left(\underset{i=1}{\mathbb{\bigotimes}} \Delta_{(\widehat{1}, \hat{i+1})}^{\otimes \vartheta_{i}}\right) \otimes \Delta_{(\widehat{1}, n-1)}^{\otimes\left(\vartheta_{n}+\cdots+\vartheta_{n-1+k}\right)}\right),
\end{aligned}
$$

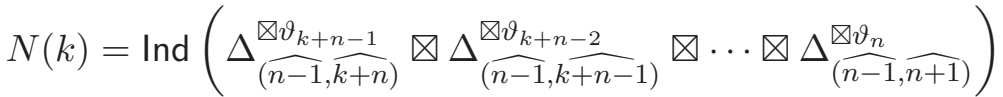

for $k=1, \ldots, n$. It follows from Lemma 4.2 that

$$
M(n) \simeq \tilde{f}_{1}^{t_{1}} \tilde{f}_{2}^{t_{2}} \cdots \tilde{f}_{n-2}^{t_{n-2}} \mathbf{1}, \quad N(n) \simeq \tilde{f}_{n}^{t_{n-1}} \tilde{f}_{n-1}^{t_{n}} \tilde{f}_{n-2}^{t_{n+1}} \cdots \tilde{f}_{1}^{t_{2 n-2}} \mathbf{1} .
$$


Since $\varepsilon_{i}(N(n))=0$ for $i=1, \ldots, n-2$, Lemma 1.8 gives

$$
\tilde{f}_{\mathbf{i}} \mathbf{1} \simeq \operatorname{hd} \operatorname{lnd}(M(n) \otimes N(n)),
$$

and this module with multiplicity one in $\operatorname{lnd}(M(n) \otimes N(n))$.

Since $\Delta_{(\widehat{1}, \widehat{n})}^{\otimes \vartheta_{n-1}}=\mathbb{C}$, Lemma 4.1 and (4.11) again give a string of surjective homomorphisms

$$
\begin{aligned}
& \operatorname{lnd}(M(n) \otimes N(n)) \simeq \operatorname{lnd}\left(M(n-1) \otimes \Delta_{(\widehat{1}, \widehat{n-1})}^{\bigotimes \vartheta_{2 n-1}} \otimes \Delta_{(\widehat{n-1}, \widehat{2 n})}^{\bigotimes \vartheta_{2 n-1}} \otimes N(n-1)\right) \\
& \rightarrow \operatorname{Ind}\left(M(n-1) \otimes \Delta_{(\widehat{1}, \widehat{2 n})}^{\otimes \vartheta_{2 n-1}} \otimes N(n-1)\right) \\
& \simeq \operatorname{lnd}\left(M(n-1) \otimes N(n-1) \otimes \Delta_{(\widehat{1}, 2 n)}^{\otimes \vartheta_{2 n-1}}\right) \\
& \vdots \\
& \rightarrow \text { Ind }\left(M(1) \otimes N(1) \otimes \Delta_{(\widehat{1}, n+2)}^{\otimes \vartheta_{n+1}} \otimes \cdots \otimes \Delta_{(\widehat{1}, \widehat{2 n})}^{\otimes \vartheta_{2 n-1}}\right)
\end{aligned}
$$

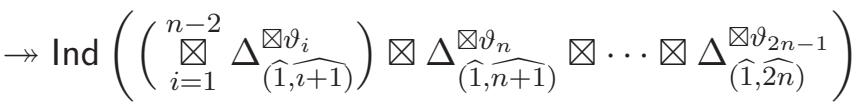

$$
\begin{aligned}
& \simeq \operatorname{lnd}\left(\underset{i=1}{2 n-1} \Delta_{(\widehat{1}, \imath+1)}^{\otimes \vartheta_{i}}\right) \text {, }
\end{aligned}
$$

which yields the desired result $\tilde{f}_{\mathbf{i}} \mathbf{1} \simeq$ hd Ind $\left(\underset{i=1}{\stackrel{\otimes}{\otimes} \Delta_{(\widehat{1}, \imath+1)}^{\otimes \vartheta_{i}}}\right)$.

As an immediate consequence of this lemma we have

Corollary 4.4. $\mathcal{N}_{n}(v)=$ hd Ind $\Delta(\mathbf{a}(v) ; n)$ for $v \in B(\infty)$, where $\mathcal{N}_{n}(v)$ is the irreducible module given in Proposition 2.3.

We now are ready to prove Theorem 3.2 ,

Proof of Theorem 3.2. Let $I_{(k)}=\{n-k+1, n-k+2, \ldots, n\}$ for $k=1, \ldots, n$. Note that $I_{(k)} \subset I_{(k+1)}$ and $\left|I_{(k)}\right|=k$. Let $U_{q}\left(\mathfrak{g}_{k}\right)$ be the subalgebra of $U_{q}(\mathfrak{g})$ generated by $e_{i}, f_{i}\left(i \in I_{(k)}\right)$ and $q^{h}\left(h \in \mathrm{P}^{\vee}\right)$, and let $\mathfrak{B}_{k}$ be the crystal obtained from $B(\infty)$ by forgetting the $i$-arrows for $i \notin I_{(k)}$. It follows from Table 1 that $U_{q}\left(\mathfrak{g}_{k}\right)$ is of type $\mathrm{X}_{k}$ when $U_{q}(\mathfrak{g})$ is of type $\mathrm{X}_{n}$ $(\mathrm{X}=\mathrm{A}, \mathrm{B}, \mathrm{C}, \mathrm{D})$. Recall the definition $\mathbf{a}_{i}$ for $\mathbf{a} \in \mathcal{S}$ and the sequences $\mathbf{s}_{k}$ given in Section 2 , Take $v \in B(\infty)$ and let $\triangle(\mathbf{a}(v))=\left\{t_{i j}\right\}$. If $i=1, \ldots, n$ for type $\mathrm{A}_{n}, \mathrm{~B}_{n}, \mathrm{C}_{n}$, then

$$
\mathbf{a}(v)_{i}=\left(t_{i, n+1-i}, t_{i, n+2-i}, \ldots, t_{i, d_{i}}\right),
$$

where $d_{i}=n$ for all $i \quad\left(\mathrm{~A}_{n}\right)$ and $d_{i}=n-1+i\left(\mathrm{~B}_{n}, \mathrm{C}_{n}\right)$. If $i=1, \ldots, n$ for type $\mathrm{D}_{n}$, then

$$
\mathbf{a}(v)_{i}= \begin{cases}\left(t_{1, n-1}\right) & \text { if } i=1, \\ \left(t_{1, n}\right) & \text { if } i=2, \\ \left(t_{i-1, n+1-i}, t_{i-1, n+2-i}, \ldots, t_{i-1, n-2+i}\right) & \text { if } i=3, \ldots, n .\end{cases}
$$


Let $M_{k}=\operatorname{Ind} \Delta(\mathbf{a}(v) ; k)$ for $k=1, \ldots, n$. Then, by Proposition 2.3, Lemma 4.3, and the choice of $I_{(k)}$, we obtain

$$
\mathcal{N}_{k}(v)=\operatorname{hd} M_{k} \quad \text { for } k=1, \ldots, n .
$$

By the construction of $\Delta(\mathbf{a}(v) ; k)$ and Lemma 4.3, we know

(i) $\varepsilon_{i}\left(M_{k}\right)=0$ for $i \in I_{(k-1)}$,

(ii) hd $M_{k}$ is irreducible and occurs with multiplicity one as a composition factor of $M_{k}$. Therefore, by Lemma 1.8 and Proposition 1.10.

$$
\begin{aligned}
\Phi(v) & =\operatorname{hd} \operatorname{lnd}\left(\left(\operatorname{hd} M_{1}\right) \otimes\left(\mathrm{hd} M_{2}\right) \otimes \cdots \otimes\left(\mathrm{hd} M_{n}\right)\right) \\
& =\operatorname{hd} \operatorname{Ind}\left(M_{1} \otimes M_{2} \otimes \cdots \otimes M_{n}\right) \\
& =\operatorname{hd} \operatorname{lnd}(\Delta(\mathbf{a}(v) ; 1) \otimes \cdots \otimes \Delta(\mathbf{a}(v) ; n)),
\end{aligned}
$$

which completes the proofs of (1) and (2).

Let $\Psi^{\lambda}: B(\lambda) \rightarrow \mathbb{B}(\lambda)$ be the canonical crystal isomorphism given by $\Psi^{\lambda}\left(\tilde{f}_{\mathbf{i}} b_{\lambda}\right)=\tilde{f}_{\mathbf{i}} \mathbf{1}$ for any sequence $\mathbf{i}$ of elements in $I$. Then the following diagram commutes:

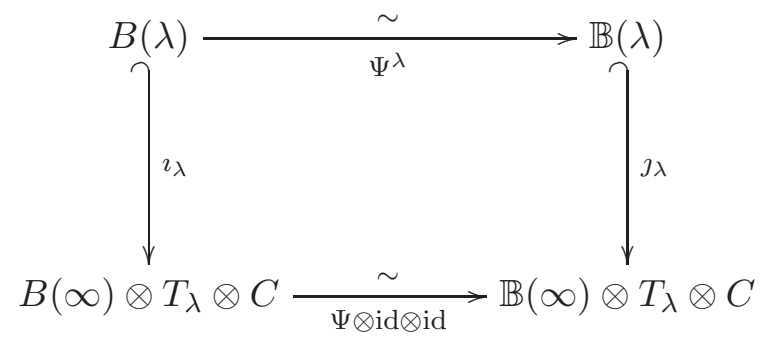

Here, $\imath_{\lambda}$ (resp. $\jmath_{\lambda}$ ) is the crystal embedding (1.1) from $B(\lambda)$ (resp. $\mathbb{B}(\lambda)$ ) to $B(\infty) \otimes T_{\lambda} \otimes C$ (resp. $\mathbb{B}(\infty) \otimes T_{\lambda} \otimes C$ ). Assume for $v \in B(\lambda)$ that $\iota_{\lambda}(v)=v^{\prime} \otimes t_{\lambda} \otimes c$ for some $v^{\prime} \in B(\infty)$, and recall that $\mathbf{a}(v)=\mathbf{a}\left(v^{\prime}\right)$. Let $\jmath_{\lambda}^{-1}$ be the inverse from $\operatorname{im}\left(\jmath_{\lambda}\right)$ to $\mathbb{B}(\lambda)$. Then

$$
\Psi^{\lambda}=\jmath_{\lambda}^{-1} \circ(\Psi \otimes \mathrm{id} \otimes \mathrm{id}) \circ \imath_{\lambda},
$$

which yields

$$
\begin{aligned}
\Psi^{\lambda}(v) & =\jmath_{\lambda}^{-1} \circ(\Psi \otimes \mathrm{id} \otimes \mathrm{id}) \circ \imath_{\lambda}(v) \\
& =\jmath_{\lambda}^{-1} \circ(\Psi \otimes \mathrm{id} \otimes \mathrm{id})\left(v^{\prime} \otimes t_{\lambda} \otimes c\right) \\
& =\jmath_{\lambda}^{-1}\left(\mathrm{hd} \operatorname{Ind}\left(\Delta\left(\mathbf{a}\left(v^{\prime}\right) ; 1\right) \otimes \cdots \otimes \Delta\left(\mathbf{a}\left(v^{\prime}\right) ; n\right)\right) \otimes t_{\lambda} \otimes c\right) \\
& =\jmath_{\lambda}^{-1}\left(\operatorname{hd} \operatorname{Ind}(\Delta(\mathbf{a}(v) ; 1) \otimes \cdots \otimes \Delta(\mathbf{a}(v) ; n)) \otimes t_{\lambda} \otimes c\right) \\
& =\operatorname{hd} \operatorname{lnd}(\Delta(\mathbf{a}(v) ; 1) \otimes \cdots \otimes \Delta(\mathbf{a}(v) ; n)) .
\end{aligned}
$$

This proves assertion (3) and concludes the proof of the main theorem (Theorem 3.2). 


\section{Acknowledgments}

Work on this paper was facilitated by a visit by S.-J. Kang, S.-j. Oh, and E. Park to the University of Wisconsin-Madison and by a visit by G. Benkart to the Korea Institute for Advanced Study. We express our gratitude to these institutions for their hospitality.

\section{REFERENCES}

1. J. Brundan and A. Kleshchev, Blocks of cyclotomic Hecke algebras and Khovanov-Lauda algebras, Invent. Math. 178 (2009), no. 3, 451-484.

2. J. Brundan and A. Kleshchev, Graded decomposition numbers for cyclotomic Hecke algebras, Adv. Math. 222 (2009), no. 6, 1883-1942.

3. J. Brundan, A. Kleshchev, and W. Wang, Graded Specht modules, J. Reine. Angew. Math. 655 (2011), $61-87$.

4. J. Brundan and C. Stroppel, Highest weight categories arising from Khovanov's diagram algebra III: category O, Represent. Theory 15 (2011), 170-243.

5. G. Cliff, Crystal bases and Young tableaux, J. Algebra 202 (1998), no. 1, 10-35.

6. J. J. Graham and G. I. Lehrer, Cellular algebras, Invent. Math. 123 (1996), no. 1, 1-34.

7. D. Hill, G. Melvin, and D. Mondragon, Representations of quiver Hecke algebras via Lyndon bases, J. Pure Appl. Algebra 216 (2012), no. 5, 1052-1079.

8. J. Hong and S.-J. Kang, Introduction to Quantum Groups and Crystal Bases, Grad. Stud. Math. 42, Amer. Math. Soc., Providence, RI, 2002.

9. J. Hong and H. Lee, Young tableaux and crystal $B(\infty)$ for finite simple Lie algebras, J. Algebra 320 (2008), no. 10, 3680-3693.

10. J. Hu and A. Mathas, Graded cellular bases for the cyclotomic Khovanov-Lauda-Rouquier algebras of type A, Adv. Math. 225 (2010), no. 2, 598-642.

11. K. Jeong, S.-J. Kang, M. Kashiwara, and D.-U. Shin, Abstract crystals for quantum generalized Kac-Moody algebras, Int. Math. Res. Not. IMRN (2007), no. 1, 1-19.

12. S.-J. Kang and M. Kashiwara, Categorification of highest weight modules via Khovanov-Lauda-Rouquier algebras, to appear in Invent. Math.; arXiv:1102.4677 (2011).

13. S.-J. Kang, M. Kashiwara, and S.-j. Oh, Categorification of highest weight modules over quantum generalized Kac-Moody algebras, to appear in Mosc. Math. J.; arXiv:1106.2635 (2011).

14. S.-J. Kang, S.-j. Oh, and E. Park, Categorification of quantum generalized Kac-Moody algebras and crystal bases, to appear in Internat. J. Math.; arXiv:1102.5165 (2011).

15. S.-J. Kang and E. Park, Irreducible modules over Khovanov-Lauda-Rouquier algebras of type $A_{n}$ and semistandard tableaux, J. Algebra 339 (2011), no. 1, 223-251.

16. M. Kashiwara, On crystal bases of the q-analogue of universal enveloping algebras, Duke Math. J. 63 (1991), no. 2, 465-516.

17. M. Kashiwara, The crystal base and Littelmann's refined Demazure character formula, Duke Math. J. 71 (1993), no. 3, 839-858.

18. M. Kashiwara and T. Nakashima, Crystal graphs for representations of the q-analogue of classical Lie algebras, J. Algebra 165 (1994), no. 2, 295-345.

19. M. Khovanov and A. Lauda, A diagrammatic approach to categorification of quantum groups I, Represent. Theory 13 (2009), 309-347. 
20. M. Khovanov and A. Lauda, A diagrammatic approach to categorification of quantum groups II, Trans. Amer. Math. Soc. 363 (2011), no. 5, 2685-2700.

21. A. Kleshchev and A. Ram, Representations of Khovanov-Lauda-Rouquier algebras and combinatorics of Lyndon words, Math. Ann. 349 (2011), no. 4, 943-975.

22. A. Lauda and M. Vazirani, Crystals from categorified quantum groups, Adv. Math. 228 (2011), no. 2, 803-861.

23. P. Littelmann, A Littlewood-Richardson rule for symmetrizable Kac-Moody algebras, Invent. Math. 116 (1994), no. 1-3, 329-346.

24. P. Littelmann, Cones, crystals, and patterns, Transform. Groups 3 (1998), no. 2, 145-179.

25. P. J. McNamara, Finite dimensional representations of Khovanov-Lauda-Rouquier algebras I: Finite type, arXiv:1207.5860 (2012).

26. R. Rouquier, 2 Kac-Moody algebras, arXiv:0812.5023 (2008).

27. B. Webster, Knot invariants and higher dimensional representation theory I: diagrammatic and geometric categorification of tensor products, arXiv:1001.2020 (2010).

Department of Mathematics, University of Wisconsin-Madison, 480 Lincoln Drive, Madison, WI 53706, USA

E-mail address: benkart@math.wisc.edu

Department of Mathematical Sciences and Research Institute of Mathematics, Seoul National University, 599 Gwanak-ro, Gwanak-gu, Seoul 151-747, Korea

E-mail address: sjkang@snu.ac.kr

Department of Mathematical Sciences, Seoul National University, 599 Gwanak-Ro, GwanakGU, SEOUl 151-747, KoreA

E-mail address: sj092@snu.ac.kr

Research Institute of Mathematics, Seoul National University, 599 Gwanak-ro, Gwanak-Gu, SEOUl 151-747, Korea

E-mail address: pwy@snu.ac.kr 